Sistemas elípticos de tipo hamiltoniano perto da ressonância 

SERVIÇO DE PÓS-GRADUAÇ̃̃O DO ICMC-USP

Data de Depósito:

Assinatura:

\title{
Sistemas elípticos de tipo hamiltoniano perto da ressonância
}

\author{
Rafael Antônio Rossato
}

Orientador: Prof. Dr. Eugenio Massa

Tese apresentada ao Instituto de Ciências Matemáticas e de Computação - ICMC-USP, como parte dos requisitos para obtenção do título de Doutor em Ciências - Matemática. VERSÃO REVISADA 
Ficha catalográfica elaborada pela Biblioteca Prof. Achille Bassi e Seção Técnica de Informática, ICMC/USP, com os dados fornecidos pelo(a) autor(a)

\begin{tabular}{|c|c|}
\hline \multirow[t]{3}{*}{ R823s } & $\begin{array}{l}\text { Rossato, Rafael Antônio } \\
\text { Sistemas elípticos de tipo hamiltoniano perto da } \\
\text { ressonância / Rafael Antônio Rossato; orientador } \\
\text { Eugenio Massa. -- São Carlos, } 2014 . \\
\quad 109 \text { p. }\end{array}$ \\
\hline & $\begin{array}{l}\text { Tese (Doutorado - Programa de Pós-Graduação em } \\
\text { Matemática)-- Instituto de Ciências Matemáticas e } \\
\text { de Computação, Universidade de São Paulo, } 2014 \text {. }\end{array}$ \\
\hline & $\begin{array}{l}\text { 1. Sistemas elípticos semilineares. 2. Problemas } \\
\text { de quase ressonância. 3. Geometria de ponto de sela. } \\
\text { 4. Aproximação finito dimensional. I. Massa, Eugenio } \\
\text {, orient. II. Título. }\end{array}$ \\
\hline
\end{tabular}




\section{Agradecimentos}

Agradeço, primeiramente, a Deus pelo dom da vida e por preparar meu caminho a cada passo desta longa caminhada, me dando saúde e força nas horas que mais precisei. Sem descansar em Teus braços, jamais teria conseguido.

A minha mãe e meus irmãos, por me ensinarem os principais valores da vida. Obrigado pela compreensão, conselhos e todo o esforço que sempre fizeram em prol de meus sonhos.

A minha esposa Chris, por me ouvir e apoiar em cada dia desta jornada, pela amizade e amor que completam a minha vida. Obrigado por compartilhar comigo o sucesso e as dificuldades, as alegrias e tristezas.

Aos amigos que fiz por estes anos em São Carlos, que tornaram o dia a dia muito mais divertido. Matemáticos ou não, do ICMC ou não, levo todos em meu coração. Um especial agradecimento aos amigos da Paróquia Nossa Senhora de Fátima, por fazer das tardes de domingo um rico alimento para minha alma.

Ao meu orientador Prof. Dr. Eugenio Massa, pelo conhecimento transmitido, exemplo de pesquisador e a paciência para lidar com as minhas dificuldades.

Aos funcionários do ICMC, em especial ao serviço de pós-graduação e aos vigilantes do instituto.

Aos membros da comissão julgadora, agradeço as críticas e sugestões que contribuíram para melhorias deste trabalho.

Agradeço à FAPESP, pelo suporte financeiro essencial a este trabalho. 
A persistência é o caminho do êxito.

Charles Chaplin 


\section{Resumo}

Neste trabalho consideramos sistemas elípticos de tipo hamiltoniano, envolvendo o operador Laplaciano, com uma parte linear dependendo de dois parâmetros e uma perturbação sublinear. Obtemos a existência de pelo menos duas soluções quando a parte linear está perto da ressonância (este fenômeno é chamado de quase ressonância). Mostramos também a existência de uma terceira solução, quando a quase ressonância é em relação ao primeiro autovalor do operador Laplaciano. No caso ressonante obtemos resultados análogos, adicionando mais uma perturbação sublinear.

Os sistemas estão associados a funcionais fortemente indefinidos, e as soluções são obtidas através do Teorema de Ponto de Sela e aproximação de Galerkin. 


\section{Abstract}

In this work we consider elliptic systems of hamiltonian type, involving the Laplacian operator, a linear part depending on two parameters and a sublinear perturbation. We obtain the existence of at least two solutions when the linear part is near resonance (this phenomenon is called almost-resonance). We also show the existence of a third solution when the almost-resonance is with respect to the first eigenvalue of the Laplacian operator. In the resonant case, we obtain similar results, with an additional sublinear term.

These systems are associated with strongly indefinite functionals, and the solutions are obtained by Saddle Point Theorem and Galerkin approximation. 


\section{Sumário}

1 Introducão 3

1.1 Apresentação dos problemas e resultados principais . . . . . . . . . . . . 3

1.2 Motivação, bibliografia e técnicas utilizadas . . . . . . . . . . . . . . 6

2 Introducão aos métodos variacionais 9

2.1 Abordagem Variacional . . . . . . . . . . . . . . . . . . . . 10

2.1 .1 Teoremas de tipo min-max . . . . . . . . . . . . . . . 11

2.2 Autovalores do Laplaciano . . . . . . . . . . . . . . . . . . . . . . . . . 14

2.3 Regularidade das solucões fracas . . . . . . . . . . . . . . . . . . . . . . 15

2.4 Sistemas de equacões elípticas . . . . . . . . . . . . . . . . . . . . . 16

2.4 .1 Os autovalores do sistema . . . . . . . . . . . . . . . . 18

3 Múltiplas solucões para um sistema elíptico quase ressonante 21

3.1 Notacões e resultados preliminares . . . . . . . . . . . . . . . . 26

3.2 Demonstracão dos principais resultados . . . . . . . . . . . . . . . . 31

3.2.1 Duas geometrias quando $a+b$ é quase ressonante inferiormente . . 31

3.2.2 Obtendo duas sequências de pontos críticos . . . . . . . . . . . 41

3.2.3 As geometrias quando $a+b$ é quase ressonante superiormente . . . 43

3.2.4 Existência de solucões no limite . . . . . . . . . . . . . . . . . 45

3.3 Quase ressonância dupla . . . . . . . . . . . . . . . . . . . . . . . 48

3.4 Condições $(\mathrm{PS})$. . . . . . . . . . . . . . . . . . . . . . . . 52

4 O caso ressonante $\quad 55$

4.1 Duas geometrias de ponto de sela no caso ressonante . . . . . . . . . . 57 
4.2 Condicões (PS) no caso ressonante . . . . . . . . . . . . . . . 61

4.3 Estimativa da perturbação sublinear $\ldots \ldots \ldots \ldots \ldots \ldots$

5 Quase ressonância com o autovalor principal $\quad 69$

5.1 Geometrias de ponto de sela quando $a+b$ é quase ressonante inferiormente 71

5.2 Existência de solucões . . . . . . . . . . . . . . . . . . . . . 77

5.3 Positividade da solucão $\widetilde{\mathbf{u}}^{j} \ldots \ldots \ldots \ldots \ldots \ldots$. . . . . . . . . . 79

5.3 .1 Lemas auxiliares . . . . . . . . . . . . . . . . . . . . . 79

5.3 .2 Mostrando solução positiva . . . . . . . . . . . . . . . 85

$\begin{array}{ll}\text { A Conceitos de Análise Funcional } & 91\end{array}$

A.1 O espaco das funcões continuamente diferenciáveis . . . . . . . . . . . . . 92

A.2 Os espacos de Hölder . . . . . . . . . . . . . . . . . . . . . . . . . 93

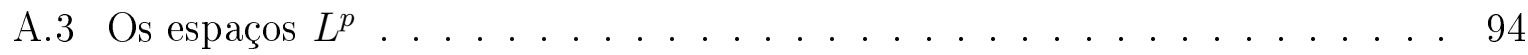

A.4 Os espaços de Sobolev . . . . . . . . . . . . . . . . . . . . . . . 96

$\begin{array}{ll}\text { B Operadores de Nemytskii } & 99\end{array}$

$\begin{array}{ll}\text { C Diferenciabilidade de funcionais em espaços de Banach } & 101\end{array}$ 


\section{Notações}

$\mathbb{R}_{+}^{N}$

$\mathbb{Z}_{0}$

$\sigma(-\Delta)$

$H_{\lambda_{k}}$

$W^{k, p}(\Omega), W_{0}^{k, p}(\Omega), H^{m}(\Omega), H_{0}^{m}(\Omega)$

$H$

$\|u\|_{H},\|u\|_{W^{k, p}},\|(u, v)\|_{H \times H},\|(u, v)\|_{W^{k, p} \times W^{k, p}}$

$\|u\|_{L^{p}},\|(u, v)\|_{L^{p} \times L^{p}}$ $\left\{\left(x_{1}, x_{2}, \ldots, x_{N}\right): x_{N}>0\right\}$

$\mathbb{Z} \backslash\{0\}$

espectro do operador $(-\Delta)$

autoespaço gerado por $\lambda_{k}$

espaços de Sobolev

$H_{0}^{1}(\Omega)$ ou $H^{1}(\Omega)$

normas nos espaços de Sobolev

normas nos espaços $L^{p}$ 


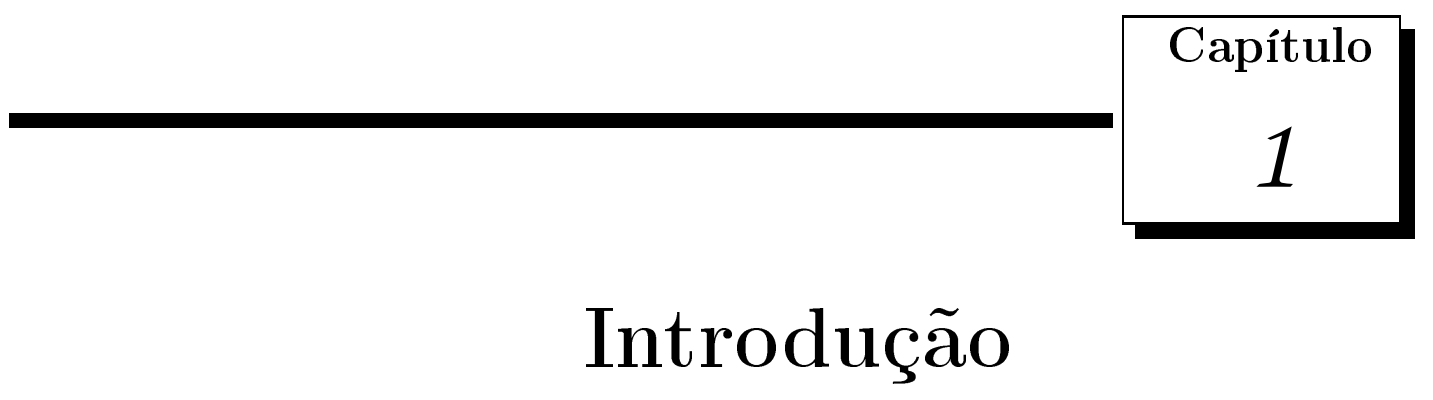

\subsection{Apresentação dos problemas e resultados principais}

Nesta tese de doutorado estudamos o seguinte sistema não linear de equações elípticas:

$$
\begin{cases}-\Delta u=a u+b v \pm\left(f_{1}(x, v)+h_{1}(x)\right) & \text { em } \Omega \\ -\Delta v=b u+a v \pm\left(f_{2}(x, u)+h_{2}(x)\right) & \text { em } \Omega \\ u(x)=v(x)=0 & \text { em } \partial \Omega\end{cases}
$$

onde $a, b \in \mathbb{R}, \Omega \subset \mathbb{R}^{N}, h_{1}, h_{2} \in L^{2}(\Omega)$ e $f_{1}, f_{2}$ são funções sublineares, em particular satisfazem a condição

$$
\begin{array}{r}
f_{i}: \Omega \times \mathbb{R} \rightarrow \mathbb{R} \text { é função de Carathéodory e existem constantes } S>0 \\
\text { e } q \in(1,2) \text {, tais que }\left|f_{i}(x, t)\right| \leq S\left(1+|t|^{q-1}\right) \text {, para cada } i=1,2 .
\end{array}
$$

Também assumimos um dos conjuntos de hipóteses sobre as funções $f_{1}, f_{2}$ e $h_{1}, h_{2}$ a seguir:

$$
\lim _{t \rightarrow \pm \infty} f_{i}(x, t)= \pm \infty, \text { uniformemente com respeito a } x \in \Omega \text { e para } i=1,2
$$


$\mathrm{Ou}$

$$
\left\{\begin{array}{l}
(i) \quad \lim _{|t| \rightarrow \infty} F_{i}(x, t)=+\infty, \text { uniformemente com respeito a } x \in \Omega, i=1,2, \\
(\text { ii }) \quad \int_{\Omega} h_{1} \phi+h_{2} \psi=0, \quad \text { para todo }(\phi, \psi) \in Z,
\end{array}\right.
$$

onde $F_{i}(x, t)=\int_{0}^{t} f_{i}(x, s) d s$ e $Z$ é um oportuno subespaço de $H_{0}^{1}(\Omega) \times H_{0}^{1}(\Omega)$.

Estaremos nos referindo ao problema (1.1) como $(1.1+)$ ou $(1.1-)$ de acordo com o sinal que aparece antes das duas não linearidades.

Nosso objetivo é obter resultados de multiplicidade de soluções, quando a parte linear de (1.1) está perto da ressonância (este fenômeno é geralmente chamado de "quase ressonância" na literatura), no sentido que $a+b$ ou $a-b$ está perto de algum autovalor de $(-\Delta)$.

É importante observar que quando os parâmetros $a \pm b \notin \sigma(-\Delta)$ é possível, através de uma abordagem variacional, demonstrar a existência de uma solução fraca de (1.1): de fato, podemos construir uma geometria de ponto de sela para o funcional associado a este sistema, obtendo um ponto crítico que corresponde a uma solução fraca do problema. Porém, os subespaços envolvidos na geometria da sela mudam quando um desses parâmetros passa de um lado para outro de um autovalor. Isso sugere que na vizinhança do autovalor (quase ressonância), deveria ser possível obter contemporaneamente as duas diferentes geometrias de sela: uma gerada pela parte linear do sistema, e outra pela perturbação sublinear. Isso nos dará duas soluções, desde que seja também possível mostrar que os níveis críticos correspondentes são distintos.

As hipóteses $(\mathbf{f})$ e $(\mathbf{F})$ garantem que a perturbação sublinear seja "suficientemente grande" (e tenha o sinal certo) para gerar a segunda geometria de sela.

Os primeiros resultados que obtemos, contidos no Capítulo 3, consideram o caso em que um dos valores $a \pm b$ é quase ressonante, enquanto o outro está a uma certa distância do espectro $\sigma(-\Delta)$. Provaremos o seguinte teorema

Teorema (Teorema [.1). Sejam $\lambda_{k}$ um autovalor de $(-\Delta), \lambda_{l}$ o primeiro autovalor acima de $a-b$ e

$$
Z=\operatorname{span}\left\{(\phi, \phi): \phi \in H_{\lambda_{k}}\right\}
$$

Suponhamos $h_{1}, h_{2} \in L^{2}(\Omega)$, e que $f_{1}, f_{2}$ satisfaçam as hipóteses (1.2) e (ff) (ou $(\overline{\boldsymbol{F}})$ ). Então 
(a) Dado $\delta>0$, existe $\varepsilon_{0}>0$ tal que, se dist $(a-b, \sigma(-\Delta))>\delta$ e $a+b \in\left(\lambda_{k}-\varepsilon_{0}, \lambda_{k}\right)$, então o problema $(1.1+)$ possui duas soluções distintas.

(b) Dado $\delta>0$, existe $\varepsilon_{1}>0$ tal que, se dist $(a-b, \sigma(-\Delta))>\delta$ e $a+b \in\left(\lambda_{k}, \lambda_{k}+\varepsilon_{1}\right)$, então o problema (1.1-) possui duas soluções distintas.

Demonstramos também o Teorema 3.2, que aborda o caso similar em que $a-b$ é quase ressonante enquanto $a+b$ está longe do espectro.

Ainda no Capítulo 3, consideramos o caso em que ambos os valores $a \pm b$ estão perto de autovalores, obtendo o seguinte resultado para o problema $(\underline{1.1}+)$ :

Teorema (Teorema 3.3). Sejam $\lambda_{k}$ e $\lambda_{l}$ dois autovalores (não necessariamente distintos) $d e(-\Delta), e$

$$
Z=\operatorname{span}\left\{(\phi, \phi): \phi \in H_{\lambda_{k}}, \quad(\phi,-\phi): \phi \in H_{\lambda_{l}}\right\}
$$

Suponhamos $h_{1}, h_{2} \in L^{2}(\Omega)$, e que $f_{1}, f_{2}$ satisfaçam as hipóteses $(1.2)$ e $(\boldsymbol{f})$ (ou $(\underline{\boldsymbol{F}})$ ). Então

(e) existe $\varepsilon_{2}>0$ tal que, se $a-b \in\left(\lambda_{l}, \lambda_{l}+\varepsilon_{2}\right)$ e $a+b \in\left(\lambda_{k}-\varepsilon_{2}, \lambda_{k}\right)$, então o problema $(1.1+)$ possui duas soluções distintas.

O Teorema 3.4 contém o resultado correspondente para o problema (1.1—).

No capítulo 4, estudamos um sistema parecido com (1.1), mas com parte linear ressonante. Neste caso, a parte quadrática do funcional não satisfaz a condição (PS) e é nula no autoespaço ressonante. Para obtermos resultados de existência e multiplicidade de soluções, como os obtidos no Capítulo 3 , precisamos de uma perturbação sublinear que tenha o duplo papel de recuperar a condição (PS) e a geometria de sela que no problema (1.1) era dada pela parte linear do sistema, sem porém cancelar os efeitos das perturbações $f_{1}, f_{2}$, necessários para obter a segunda geometria de sela. Nestas condições, conseguimos demonstrar resultados análogos aos do Capítulo 3 (veja os Teoremas 4.1, 4.3 e 4.4).

No Capítulo [5, voltamos a considerar o sistema (1.1), no caso particular em que a quase ressonância é em relação ao primeiro autovalor do Laplaciano $\lambda_{1}$. Neste caso será possível obter uma terceira solução e, inclusive, uma informação sobre o sinal de duas das três soluções (veja os Teoremas 5.1 e 5.2). 
O Capítulo2 e os apêndices, apresentam um resumo dos teoremas variacionais clássicos e resultados da análise funcional que utilizamos ao longo do trabalho.

\subsection{Motivação, bibliografia e técnicas utilizadas}

Problemas de quase ressonância têm sido intensamente estudados, em particular no caso escalar

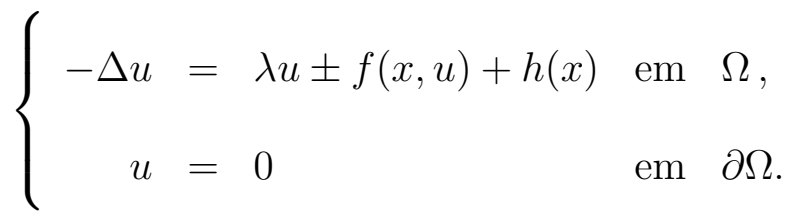

O caso em que a quase ressonância é em relação ao primeiro autovalor foi estudado por diversos autores, começando com o trabalho de Mawhin e Schmitt [MS90], onde é considerado o problema em dimensão um usando técnicas de bifurcação do infinito e teoria do grau. Citamos ainda [BL89, LR90], onde os autores também consideram o caso em dimensão um, e [CMN92, CdF93], que lidam com o problema em dimensão maior. Todos estes trabalhos utilizam a teoria de bifurcação. Resultados para autovalores acima do primeiro, foram obtidos em [LR90], novamente usando bifurcação do infinito e teoria do grau, mas somente para o caso em dimensão um, no qual todos os autovalores são simples.

Em [RS97, MRS97], foi estudado o mesmo tipo de problema, mas de um ponto de vista variacional, e foram encontradas ao menos três soluções quando a quase ressonância é em relação ao primeiro autovalor, sob condições que são basicamente a nossa hipótese (F). A abordagem variacional foi depois explorada em [MP02] para obter um resultado similar para o operador p-Laplaciano (veja também [DNM01]).

O trabalho que nos inspirou para começar a indagar os problemas desta tese é dPM08], onde os autores consideram o problema (1.3) e, para $h \in L^{2}(\Omega), f$ sublinear e satisfazendo uma condição análoga a ( $(\mathbf{f})$ (ou $(\mathbf{F})$ ), obtiveram, através de métodos variacionais, o seguinte resultado (ainda nos referimos a este problema como $(\underline{1.3}+)$ ou (1.3-), baseado no sinal antes da não linearidade $f$ ):

a) existe $\varepsilon_{0}>0$, tal que se $\lambda \in\left(\lambda_{k}-\varepsilon_{0}, \lambda_{k}\right)$, existem duas soluções para o problema $(1.3+)$;

b) existe $\varepsilon_{1}>0$, tal que se $\lambda \in\left(\lambda_{k}, \lambda_{k}+\varepsilon_{1}\right)$ existem duas soluções para o problema 
$(1.3-)$.

Algumas melhorias ao resultado de dPM08 foram obtidas em [KT11b, no sentido de considerar condições um pouco mais fracas sobre a não linearidade $f$ e o termo $h$ : no lugar da condição $\int_{\Omega} h \phi=0$, para $\phi \in H_{\lambda_{k}}$ (que corresponde à nossa (F-ii)), é imposta uma condição de tipo Landesman-Lazer. Isto permite, em alguns casos, que a função $h$ tenha uma componente limitada (não necessariamente zero) no autoespaço quase ressonante.

O resultado em dPM08 foi obtido encontrando duas geometrias de ponto de sela para o funcional associado ao problema (1.3), uma com um enlace de ordem $k-1$ e outra com enlace de ordem $k+m-1$, onde $m$ é a multiplicidade do autovalor $\lambda_{k}$. Em seguida, é mostrado que estas soluções são distintas, uma vez que possuem níveis críticos distintos.

No caso do sistema (1.1), precisamos lidar com um funcional fortemente indefinido, no sentido que existem dois subespaços do seu espaço de definição, ambos de dimensão infinita, onde a parte principal do funcional é ilimitada superiormente em um deles e ilimitada inferiormente no outro. Desta forma, os Teoremas de enlace clássicos não podem ser utilizados a fim de encontrar pontos críticos. Para contornar esse problema, escolhemos recorrer a uma técnica de aproximação finito dimensional (a saber, o Método de Galerkin): primeiramente encontramos soluções de problemas finito dimensionais, para os quais podemos usar os teoremas de enlace clássicos, em seguida tomamos o limite na dimensão de tais problemas e mostramos que o limite é um dos pontos críticos que procuramos. As referências [MR06, dFdÓR04], são exemplos de trabalhos que utilizam esta técnica de aproximação.

Em relação a problemas quase ressonantes para sistemas, citamos AS12, OT09, ST10, KT11a. Os três primeiros consideram sistemas variacionais de tipo gradiente, nos quais a parte principal do funcional associado ao problema tem um subespaço negativo de dimensão finita, e portanto muitas técnicas utilizadas para o caso escalar podem ser aplicadas diretamente.

Por outro lado, em [KT11a] os autores consideram um sistema, diferente do nosso (1.1), mas para o qual, como no nosso caso, o funcional associado é fortemente indefinido. Eles demonstram a existência de duas soluções no caso quase ressonante, sob condições semelhantes às de [KT11b], utilizando teoremas de enlace generalizados para funcionais fortemente indefinidos (ver [Rab86, MMP94]). Observamos que eles consideram um único parâmetro $\mu$ para levar o sistema perto da ressonância, enquanto nós estudamos o com- 
portamento do sistema (1.1) com respeito a ambos os parâmetros $a$ e $b$.

Vale destacar que tanto [KT11a quanto dPM08 precisaram de duas demonstrações diferentes para o caso acima e abaixo do autovalor, uma vez que a situação é assimétrica: em [dPM08], em particular, o subespaço ressonante é parte do subespaço negativo (de dimensão finita) da parte quadrática do funcional num caso, enquanto é parte do subespaço positivo (de dimensão infinita) no outro caso; em [KT11a], mesmo sendo os dois subespaços de dimensão infinita, eles realizam papéis diferentes no teorema de enlace generalizado.

Com a nossa abordagem via método de Galerkin, não há mais a necessidade de duas demonstrações diferentes, pois as geometrias de ponto de sela tornam-se análogas nos dois casos (compare as Seções 3.2.1 e 3.2.3).

Os resultados do Capítulo 5, sobre quase ressonância com o primeiro autovalor, estão na linha de [RS97, MRS97]: ainda utilizamos a técnica de Galerkin para encontrar duas geometrias de sela semelhantes com as do Capítulo 3, Em seguida, seguindo as ideias de [RS97], obtemos uma informação sobre o sinal de uma das duas soluções obtidas; a este ponto será fácil, por simetria, obter uma terceira solução tendo os sinais opostos. 


\section{Capítulo}

2

\section{Introdução aos métodos variacionais}

Neste capítulo apresentamos algumas definições e resultados clássicos que serão utilizados ao longo do trabalho. Para mais detalhes a respeito destes resultados, ver por exemplo as referências [Eva98, Rab86, AM07, Bre83, Str90, Wil96, GT01.

Consideremos a seguinte equação

$$
\left\{\begin{array}{l}
-\Delta u=f(x, u), \text { em } \Omega \\
u=0, \text { em } \partial \Omega
\end{array}\right.
$$

onde $\Omega$ é um domínio limitado em $\mathbb{R}^{N}$. Suponhamos também que $f: \Omega \times \mathbb{R} \rightarrow \mathbb{R}$ seja uma função de Carathéodory, isto é, $f$ satisfaz as propriedades

- para cada $s \in \mathbb{R}$ fixado, a função $x \mapsto f(x, s)$ é Lebesgue mensurável em $\Omega$;

- para quase todo $x \in \mathbb{R}$, a função $s \mapsto f(x, s)$ é contínua em $\mathbb{R}$.

No que segue, estaremos denotando por $H$ o espaço de Sobolev $H_{0}^{1}(\Omega)$. Dizemos que $u$ é solução clássica de (2.1), se $u \in \mathcal{C}^{2}(\Omega) \cap \mathcal{C}^{0}(\bar{\Omega})$ e satisfaz a equação em (2.1). Dizemos que $u \in H$ é solução fraca de (2.1), se

$$
\int_{\Omega} \nabla u \nabla v=\int_{\Omega} f(x, u) v, \text { para todo } v \in H .
$$

Em verdade, toda solução clássica é uma solução fraca. De fato, basta multiplicar a equação (2.1) por uma função arbitrária $v \in H$ e integrar por partes. A recíproca também 
é verdadeira quando a solução fraca for suficientemente regular.

\subsection{Abordagem Variacional}

Consideremos o funcional

$$
I(u)=\frac{1}{2} \int_{\Omega}|\nabla u|^{2}-\int_{\Omega} F(x, u)
$$

onde $F(x, s)=\int_{0}^{s} f(x, t) d t$. Se este funcional está bem definido em $H$ e é de classe $\mathcal{C}^{1}(H, \mathbb{R})$ (ver apêndice $(\mathbf{C})$, então temos uma relação entre as soluções fracas de (2.1) e os pontos críticos de $I$, isto é, os pontos $\bar{u} \in H$ tais que a derivada de Fréchet $I^{\prime}(\bar{u})=0$.

Os chamados Métodos Variacionais consistem em aproveitar esta relação para obter soluções de (2.1), através do estudo da geometria do funcional (2.3).

O exemplo mais simples desta técnica, consiste em procurar um mínimo do funcional I. A existência de um mínimo global poderá ser garantida pelo teorema a seguir.

Teorema 2.1. Sejam $E$ um espaço de Banach reflexivo e $I: E \rightarrow \mathbb{R}$, tais que

- I é coercivo, isto é, $I(x) \rightarrow \infty$ se $\|x\|_{E} \rightarrow \infty$;

- I é sequencialmente fracamente semicontínuo inferiormente, isto é, se $\left\{u_{n}\right\} \subset E$ e $u_{n} \rightarrow u$ então $I(u) \leq \liminf _{n \rightarrow \infty} I\left(u_{n}\right)$.

Então existe $u_{0} \in E$, tal que $I\left(u_{0}\right)=\inf _{u \in E} I(u)>-\infty$.

Ideia da demonstração. Pela coercividade podemos provar que uma sequência que realiza o ínfimo deve ser limitada, logo pela reflexividade, possui uma subsequência fracamente convergente para algum $u_{0} \in E$. Finalmente pela semicontinuidade inferior, segue $I\left(u_{0}\right)=$ $\inf _{u \in E} I(u)$.

Em muitas aplicações o funcional $I$ pode não possuir um mínimo global, ou podemos estar interessados em encontrar mais pontos críticos. Por isso torna-se interessante estudar outras técnicas que garantam a existência de pontos críticos. Veremos na seção a seguir. 


\subsubsection{Teoremas de tipo min-max}

Dado um espaço de Banach $E$ e um funcional $I \in \mathcal{C}^{1}(E, \mathbb{R})$, definamos para qualquer $c \in \mathbb{R}$, os conjuntos

$$
\begin{gathered}
K_{c}=\left\{u \in E \text { tal que } I(u)=c \text { e } I^{\prime}(u)=0\right\}, \\
A_{c}=\{u \in E \text { tal que } I(u) \leq c\} .
\end{gathered}
$$

Assim podemos definir o conceito de sequência de Palais-Smale e a condição de PalaisSmale.

Definição 2.2. Sejam $E$ um espaço de Banach e $I \in \mathcal{C}^{1}(E, \mathbb{R})$. Dizemos que $\left\{u_{n}\right\} \subset E$ é uma sequência $(P S)_{\beta}$ (de Palais-Smale no nivel $\beta \in \mathbb{R}$ ) se

$$
I\left(u_{n}\right) \rightarrow \beta \text { e } I^{\prime}\left(u_{n}\right) \rightarrow 0 \text { em } E^{\prime}, \text { quando } n \rightarrow \infty
$$

onde $E^{\prime}$ é o dual do espaço E.

Dizemos que I satisfaz a condição $(P S)_{\beta}$, se toda sequência $(P S)_{\beta}$ possui uma subsequência convergente.

Dizemos que I satisfaz a condição (PS), se I satisfaz a condição $(P S)_{\beta}$ para todo $\beta \in \mathbb{R}$.

A condição de Palais-Smale, primeiramente introduzida por Palais e Smale em [PS64, Pal63, Sma64, é importante pois implica na compacidade dos conjuntos $K_{c}$. Em particular, se o funcional é de classe $\mathcal{C}^{1}$, ela garante que se provarmos a existência de uma sequência de "pontos quase críticos no nível $c$ " (isto é uma sequência $(P S)_{c}$ ), então existe um ponto crítico no nível $c$.

A principal ferramenta para provar a existência de um ponto crítico é o Lema de Deformação a seguir, cuja demonstração pode ser encontrada em [Rab86].

Lema 2.3 (Lema de Deformação). Sejam E um espaço de Banach e $I \in \mathcal{C}^{1}(E, \mathbb{R})$. Suponhamos que I satisfaz a condição $(P S)$ e seja $c \in \mathbb{R}$, tal que $K_{c}=\emptyset$. Para qualquer $\bar{\varepsilon}>0$, existem $\varepsilon \in(0, \bar{\varepsilon})$ e $\eta \in \mathcal{C}([0,1] \times E, E)$, tais que

(a) $\eta(0, u)=u$ para todo $u \in E$;

(b) $\eta(t, u)=u$ para todo $(t, u)$ tal que $I(u) \notin[c-\bar{\varepsilon}, c+\bar{\varepsilon}]$; 
(c) $\eta(t, \cdot): E \rightarrow E$ é um homeomorfismo para todo $t \in[0,1]$;

(d) $\eta\left(1, A_{c+\varepsilon}\right) \subset A_{c-\varepsilon}$.

Utilizando este Lema de Deformação, podemos provar a existência de pontos críticos considerando a geometria do funcional. A ideia em geral é:

- considerar uma classe $\Gamma$ de subconjuntos de $E$,

- definir $c=\inf _{A \in \Gamma} \sup _{u \in A} I(u)$,

- dar condições tais que se possa construir a deformação $\eta$, tal que $\eta(1, A) \in \Gamma$ para todo $A \in \Gamma$,

- obter uma contradição entre a caracterização infsup de $c$ e o fato que, se $K_{c}$ é vazio, podemos encontrar $A \in \Gamma$, tal que $\sup _{u \in \eta(1, A)} I(u)<c$.

O exemplo mais clássico, do qual apresentamos a demonstração, é o Teorema do Passo da Montanha (ver [AR73]).

Teorema 2.4 (Teorema do Passo da Montanha). Sejam E um espaço de Banach e I $\in$ $\mathcal{C}^{1}(E, \mathbb{R})$ satisfazendo

- $I(0)=0$;

- existem $r, \alpha>0$ tais que $I(u) \geq \alpha$, para todo $\|u\|_{E}=r$;

- existe $e \in E$, tal que $\|e\|_{E}>r$ e $I(e)<0$.

Além disso, sejam

- $\Gamma=\left\{\gamma \in \mathcal{C}^{0}([0,1], E), \quad\right.$ tal que $\quad \gamma(0)=0 \quad$ e $\left.\gamma(1)=e\right\}$;

- $c=\inf _{\gamma \in \Gamma} \sup _{t \in[0,1]} I(\gamma(t))$.

Então $c \geq \alpha>0$ e existe uma sequência $(P S)_{c}$. Se I satisfaz a condição $(P S)_{c}$, então c é um valor crítico para $I$. 
Ideia da demonstração. Uma vez que $\|0\|_{E}<\rho<\|e\|_{E}$, para toda $\gamma \in \Gamma$ existe $\bar{t} \in[0,1]$, tal que $\|\gamma(\bar{t})\|_{E}=r$ e então

$$
\sup _{t \in[0,1]} I(\gamma(t)) \geq \alpha, \quad \forall \gamma \in \Gamma
$$

Suponhamos $K_{c}=\emptyset$ e escolhamos $\bar{\varepsilon}<\frac{\alpha}{2}$. Pelo Lema de Deformação, existiria $\varepsilon<\bar{\varepsilon}$ e uma deformação $\eta$, tais que $\eta\left(1, A_{c+\varepsilon}\right) \subseteq A_{c-\varepsilon}$. Então escolhendo $\gamma_{\varepsilon} \in \Gamma$, tal que

$$
\sup _{u \in \gamma_{\varepsilon}([0,1])} I(u)<c+\varepsilon
$$

e considerando $\eta\left(1, \gamma_{\varepsilon}(\cdot)\right)$,

- pela propriedade $(c)$ do Lema de Deformação, teríamos $\eta\left(1, \gamma_{\varepsilon}(\cdot)\right) \in \mathcal{C}^{0}([0,1], E)$;

- pela propriedade $(b)$ do Lema de Deformação, uma vez que $c-\bar{\varepsilon}>\frac{\alpha}{2}>0$, obteríamos $\eta\left(1, \gamma_{\varepsilon}(\cdot)\right)=\gamma_{\varepsilon}(0)=0$ e $\eta\left(1, \gamma_{\varepsilon}(1)\right)=\gamma_{\varepsilon}(1)=e ;$

- pela propriedade $(d)$ do Lema de Deformação, $\sup _{u \in \eta\left(1, \gamma_{\varepsilon}([0,1])\right)} I(u) \leq c-\varepsilon$;

então concluiríamos que $\eta\left(1, \gamma_{\varepsilon}(\cdot)\right) \in \Gamma$ e então a última desigualdade seria uma contradição com a definição de $c$.

Abaixo enunciamos o Teorema do Ponto de Sela que utilizamos para obter os principais resultados deste trabalho.

Teorema 2.5 (Teorema do Ponto de Sela). Sejam $E=V \oplus X$ um espaço de Banach com $\operatorname{dim}(V)<\infty$, e $I \in \mathcal{C}^{1}(E, \mathbb{R})$ um funcional satisfazendo a condição (PS). Se D é uma vizinhança limitada de 0 em $V$, tal que

$$
\begin{array}{ll}
I(u) \geq b, & \forall u \in X, \\
I(u) \leq a, & \forall u \in \partial D,
\end{array}
$$

com $a<b$, então definindo

$$
c=\inf _{h \in \Gamma} \sup _{u \in \bar{D}} I(h(u))
$$

onde $\Gamma=\{h \in \mathcal{C}(\bar{D}, \mathbb{R}): h(u)=u, \forall u \in \partial D\}$, temos que $c \geq b$ e existe um ponto crítico de I no nível $c$. 
Quando estiverem satisfeitas as condições (2.4) e (2.5), dizemos que o funcional possui uma geometria de ponto de sela.

\subsection{Autovalores do Laplaciano}

No estudo de equações do tipo (2.1), é importante considerar o conjunto $\sigma(-\Delta) \subseteq \mathbb{C}$, que é o conjunto dos $\lambda$, tais que existe solução não trivial do problema

$$
\begin{cases}-\Delta u(x)=\lambda u(x) & x \in \Omega, \\ u=0 & x \in \partial \Omega .\end{cases}
$$

O conjunto $\sigma(-\Delta)$ é chamado espectro do operador Laplaciano, cada $\lambda \in \sigma(-\Delta)$ é dito autovalor, e as correspondentes soluções não triviais de (2.6) são ditas autofunções associadas ao autovalor $\lambda$.

Resumimos abaixo alguns fatos conhecidos a respeito do espectro do Laplaciano, quando $\Omega$ é limitado.

- Os autovalores são todos reais, não negativos e formam uma conjunto discreto ilimitado superiormente.

- Para cada autovalor $\lambda$, o conjunto $H_{\lambda}$ das autofunções associadas é um subespaço de dimensão finita de $H$. A dimensão deste autoespaço é dita multiplicidade do autovalor. Autovalores distintos possuem autoespaços ortogonais em $L^{2}$ e em $H$, Além disso as autofunções são não nulas q. t. p. em $\Omega$, de classe $\mathcal{C}^{\infty}(\Omega)$ e, se $\Omega$ é regular, são também $\mathcal{C}^{\infty}(\bar{\Omega})$.

- Existe um primeiro autovalor, que é positivo, simples, isto é, de multiplicidade 1, e principal isto é, todas as autofunções correspondentes são múltiplas de uma função estritamente positiva em $\Omega$.

- Ordenamos os autovalores numa sequência não decrescente $\left\{\lambda_{k}\right\}_{k=1,2 \ldots}$, repetindo cada um deles de acordo com sua multiplicidade, e denotemos por $\phi_{k}$ um gerador do correspondente autoespaço, tomado tal que $\phi_{1}>0,\left\|\phi_{k}\right\|_{L^{2}}=1 \mathrm{e}\left\langle\phi_{k}, \phi_{h}\right\rangle_{L^{2}}=0$ para $k \neq h$. Denotemos o autoespaço associado a um autovalor $\lambda_{k}$ por $H_{\lambda_{k}}$. 
- A sequência das autofunções escolhidas acima é uma base ortogonal para o espaço $H$, isto é, qualquer $u \in H$ pode ser escrito de modo único como $u=\sum_{i=1}^{+\infty} c_{i} \phi_{i}$, com $c_{i} \in \mathbb{R}$, para todo $i \in \mathbb{N}$. Além disso $\|u\|_{L^{2}}^{2}=\sum_{i=1}^{+\infty} c_{i}^{2}$ e $\|\nabla u\|_{L^{2}}^{2}=\sum_{i=1}^{+\infty} \lambda_{i} c_{i}^{2}$.

Um resultado clássico a respeito da relação da função $f(x, u)$ de (2.1) e o espectro do operador Laplaciano, é o seguinte:

Teorema 2.6 (Alternativa de Fredholm para o problema de Dirichlet). Seja $f(x, u)=$ $\lambda u+h(x)$ com $h \in L^{2}(\Omega)$, então temos

- se $\lambda \notin \sigma(-\Delta)$, então existe uma única solução do problema (2.1);

- se $\lambda \in \sigma(-\Delta)$, então existe solução se, e somente se, $\langle h, \phi\rangle_{L^{2}}=0$, para todo $\phi \in H_{\lambda}$; além disso se $\bar{u}$ é uma solução, então $\bar{u}+w$ é uma solução também se, e somente se, $w \in H_{\lambda}$.

\subsection{Regularidade das soluções fracas}

Nesta seção apresentamos algumas condições suficientes para que uma solução fraca seja também uma solução clássica. Começamos definindo o conceito de regularidade de um subconjunto $\Omega$ de $\mathbb{R}^{N}$.

Definição 2.7. Um aberto limitado $\Omega$ em $\mathbb{R}^{N}$ é dito de classe $\mathcal{C}^{k, \alpha}$ (ou $\mathcal{C}^{k}$ se $\alpha=0$ ), com $0 \leq \alpha \leq 1$, se para cada ponto $x_{0} \in \partial \Omega$, existe uma bola $B=B\left(x_{0}\right)$ e uma aplicação bijetora $\psi$ de $B$ em $D \subset \mathbb{R}^{N}$, tal que:

$$
\psi(B \cap \Omega) \subset \mathbb{R}_{+}^{N} ; \quad \psi(B \cap \partial \Omega) \subset \partial \mathbb{R}_{+}^{N} ; \quad \psi \in \mathcal{C}^{k, \alpha}(B) ; \quad \psi^{-1} \in \mathcal{C}^{k, \alpha}(D),
$$

onde $\mathbb{R}_{+}^{N}$ é um semiespaço em $\mathbb{R}^{N}$.

Enunciamos agora um resultado de regularidade de soluções fracas de problemas elípticos que será usado no Capítulo 5, Outros resultados sobre regularidade de soluções fracas e estimativas $L^{p}$ podem ser encontrados em [ADN59, Bre83, GT01, Str90].

Teorema 2.8 (do Teorema 8.2 de $\operatorname{Agm59}$ ). Sejam $\Omega$ um aberto limitado de $\mathbb{R}^{N}$ de classe $\mathcal{C}^{2}, K>0$ e $1<p<\infty$. 
Então existe uma constante $C$, dependendo apenas de $N, \Omega, p, K$ tal que, se $u \in L^{q}(\Omega)$, com $1<q<\infty$, é solução fraca de

$$
\begin{cases}-\Delta u+c u=f(x), & \text { em } \Omega, \\ u(x)=0, & \text { em } \partial \Omega\end{cases}
$$

com $|c| \leq K$ e $f \in L^{p}(\Omega)$, então $u \in W^{2, p}(\Omega) \cap W_{0}^{1, p}(\Omega)$ e

$$
\|u\|_{W^{2, p}} \leq C\left(\|u\|_{L^{p}}+\|f\|_{L^{p}}\right) .
$$

\subsection{Sistemas de equações elípticas}

Neste trabalho lidamos com sistemas não lineares de equações elípticas, e nossos resultados serão obtidos através de métodos variacionais como os expostos na Seção 2.1, Entretanto, ao lidar com sistemas, podem aparecer fenômenos que não estão presentes no caso de uma única equação. Em particular, para poder associar ao problema (2.1) o funcional (2.3), é suficiente que a função $F(x, u(x))$ seja integrável para $u \in H$, enquanto no caso de sistemas nem sempre é possível associar o problema a um funcional. Diremos que o problema é variacional, quando isto for possível. Os sistemas não variacionais podem ser tratados com outras técnicas existentes na literatura, como por exemplo a teoria do grau e técnicas de blow-up, entre outras. Veja por exemplo dF00] e dF08].

Consideremos sistemas de dois problemas elípticos envolvendo o operador Laplaciano, na forma

$$
\begin{cases}-\Delta u=f(x, u, v) ; & \text { em } \quad \Omega \\ -\Delta v=g(x, u, v) ; & \text { em } \quad \Omega \\ u=v=0 ; & \text { em } \quad \partial \Omega\end{cases}
$$

onde $\Omega \subseteq \mathbb{R}^{N}$. As propriedades do problema dependem do tipo de acoplamento que existe entre as equações.

Um primeiro caso, chamado de tipo gradiente, é quando existe uma função $F: \bar{\Omega} \times$ $\mathbb{R} \times \mathbb{R} \rightarrow \mathbb{R}$, tal que

$$
F_{u}(x, u, v)=f(x, u, v) \quad \text { e } \quad F_{v}(x, u, v)=g(x, u, v),
$$


onde $F_{u}=\frac{\partial F}{\partial u}$ e $F_{v}=\frac{\partial F}{\partial v}$. Neste caso, se $F(x, u(x), v(x))$ é integrável para $u, v \in H$, o problema é variacional e o funcional associado é

$$
\Phi(u, v)=\frac{1}{2} \int_{\Omega}|\nabla u|^{2}+\frac{1}{2} \int_{\Omega}|\nabla v|^{2}-\int_{\Omega} F(x, u, v),
$$

definido no produto cartesiano $H \times H$. A condição de integrabilidade acima será satisfeita se impusermos a seguinte hipótese:

$$
\begin{aligned}
\left|F_{u}(x, u, v)\right| & \leq C\left(1+|u|^{p}+|v|^{p}\right), \\
\left|F_{v}(x, u, v)\right| & \leq C\left(1+|u|^{p}+|v|^{p}\right),
\end{aligned}
$$

para alguma constante $C>0$ e, se $N \geq 3, p \leq 2^{*}-1$ sendo $2^{*}=\frac{2 N}{N-2}$.

Observemos que a parte principal do funcional (2.11) é o quadrado da norma do vetor $(u, v)$ no produto cartesiano $H \times H$, exatamente como em (2.3). Isso permite a aplicação direta das mesmas técnicas variacionais utilizadas no caso escalar.

Os sistemas com os quais lidamos neste trabalho, pertencem a uma outra importante e muito estudada classe de sistemas elípticos variacionais, os chamados de tipo hamiltoniano, isto é, para os quais existe uma função $\mathcal{H}: \bar{\Omega} \times \mathbb{R} \times \mathbb{R} \rightarrow \mathbb{R}$, tal que

$$
\mathcal{H}_{v}(x, u, v)=f(x, u, v) \quad \text { e } \quad \mathcal{H}_{u}(x, u, v)=g(x, u, v) .
$$

Neste caso, se $\mathcal{H}(x, u(x), v(x))$ é integrável para $u, v \in H$, o funcional associado é

$$
\Phi(u, v)=\int_{\Omega} \nabla u \nabla v-\int_{\Omega} \mathcal{H}(x, u, v)
$$

ainda definido em $H \times H$.

Este tipo de problema tem sido objeto de intensa pesquisa, começando com os trabalhos de Clément, de Figueiredo e Mitidieri [CdFM92], Hulshof e van der Vorst [HvdV93].

O funcional (2.15) possui uma estrutura diferente com respeito a (2.3) e (2.11), pois sua parte principal não é uma norma, e sim o produto interno entre os gradientes das incógnitas: em particular, o espaço $H \times H$ pode ser decomposto em dois subespaços, ambos de dimensão infinita, tais que a parte principal de $\Phi$ seja ilimitada superiormente em um deles e ilimitada inferiormente no outro. Dizemos então que $\Phi$ é fortemente indefinido. 
Por esta razão, torna-se impossível a aplicação direta dos teoremas descritos na Seção 2.1.1, pois eles sempre requerem que um destes dois subespaços tenha dimensão finita (veja por exemplo a hipótese sobre o subespaço $V$ no Teorema 2.5). Para lidar com esta dificuldade nós recorreremos a uma técnica de aproximação finito dimensional (o chamado Método de Galerkin): encontraremos inicialmente soluções de problemas finito dimensionais, para os quais podemos usar os teoremas de variacionais clássicos, e em seguida passaremos ao limite na dimensão de tais problemas e mostramos que estas soluções convergem para um ponto crítico de $\Phi$. As referências [AFdÓR04, MR06], são exemplos de trabalhos que utilizam esta técnica de aproximação.

Existem também teoremas que garantem a existência de pontos críticos para funcionais fortemente indefinidos (ver [Rab86]). Os trabalhos [Mas07, Fel93], utilizam destes teoremas.

\subsubsection{Os autovalores do sistema}

Consideremos o problema de autovalor

$$
\begin{cases}-\Delta u=\lambda v, & \text { em } \Omega, \\ -\Delta v=\lambda u, & \text { em } \Omega, \\ u=v=0, & \text { em } \partial \Omega .\end{cases}
$$

Somando e subtraindo as duas equações acima, e denotando por $s=u+v$ e $d=u-v$, temos

$$
\begin{cases}-\Delta s=\lambda s, & \text { em } \Omega \\ -\Delta d=-\lambda d, & \text { em } \Omega \\ s=d=0, & \text { em } \partial \Omega .\end{cases}
$$

Notemos que se $\lambda=\lambda_{k} \in \sigma(-\Delta)$, então $u+v=c \phi_{k}$, para alguma autofunção $\phi_{k} \mathrm{e}$ $u-v=0$ (pois $-\lambda \notin \sigma(-\Delta)$ ). Por outro lado, se $-\lambda=\lambda_{k} \in \sigma(-\Delta)$, temos $u-v=c \phi_{k}$, e $u+v=0$ (pois $\lambda \notin \sigma(-\Delta))$.

Segue disto que os autovalores do sistema (2.16) são

- $\lambda_{k}, k=1,2 \ldots$, com os pares $\left(\phi_{k}, \phi_{k}\right), \phi_{k} \in H_{\lambda_{k}}$, como as autofunções correspondentes, 
- $-\lambda_{k}, k=1,2 \ldots$, com os pares $\left(\phi_{k},-\phi_{k}\right), \phi_{k} \in H_{\lambda_{k}}$, como as autofunções correspondentes. 


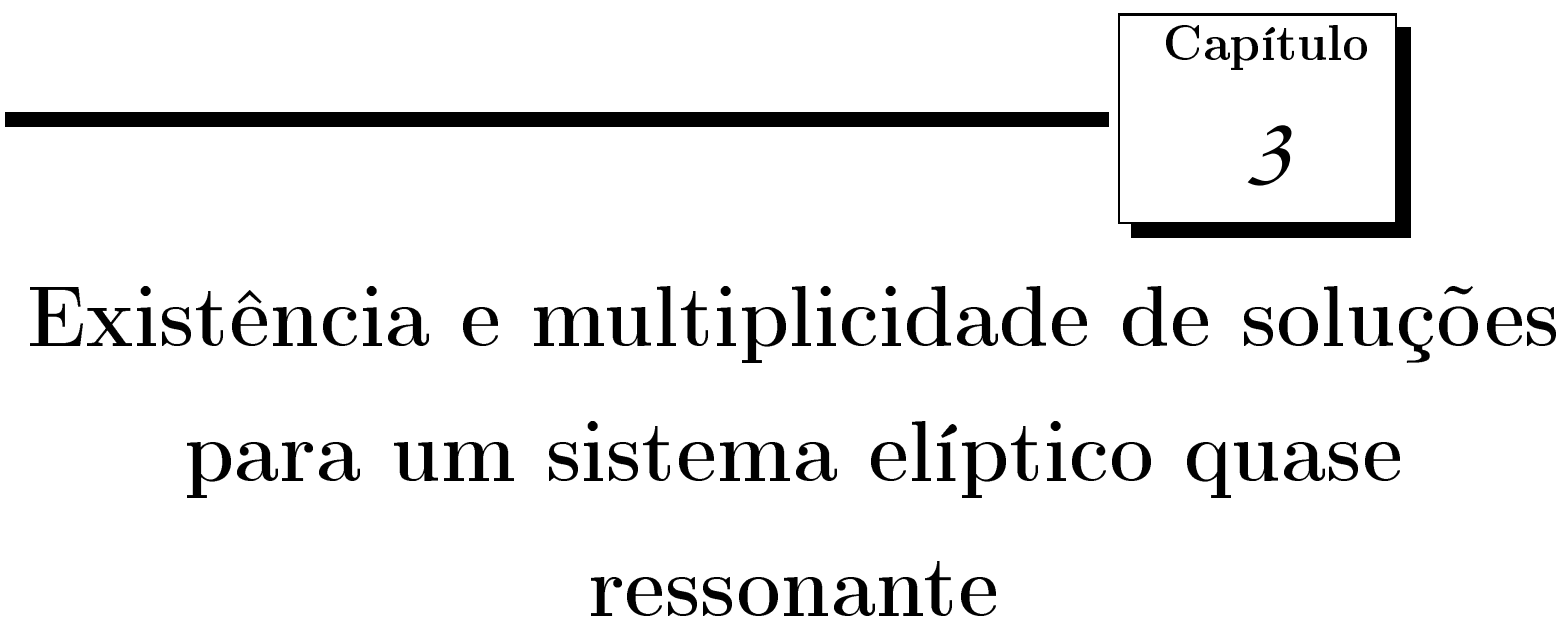

Neste capítulo obtemos vários resultados de multiplicidade de soluções para o sistema elíptico (1.1), perto da ressonância.

Como já comentamos no Capítulo (1), a principal motivação deste capítulo é o artigo dPM08, onde é demonstrado um resultado semelhante para o caso escalar (1.3).

Como observado em [dPM08, a possibilidade de encontrar duas soluções é sugerida pela seguinte observação: se $\lambda \notin \sigma(-\Delta)$, sempre existe uma solução do problema (1.3) (para $\lambda>\lambda_{1}$ ), que pode ser obtida pelo Teorema do Ponto de Sela. Entretanto, na geometria do ponto de sela, a dimensão do espaço negativo é diferente quando $\lambda$ está acima ou abaixo do autovalor $\lambda_{k}$. Isto significa que a geometria da parte quadrática do funcional muda quando $\lambda$ passa de baixo para cima do autovalor $\lambda_{k}$, e então é possível obter as duas geometrias de ponto de sela ao mesmo tempo, se $\lambda$ está perto de $\lambda_{k}$ e a perturbação $f$ fizer o funcional mudar de sinal no autoespaço $H_{\lambda_{k}}$. Para obter isto, o termo $\pm f$ em (1.3) precisa ter sinal adequado (diferente se considerarmos $\lambda$ abaixo ou acima de $\lambda_{k}$ ) e ser "suficientemente grande", no sentido das hipóteses $(\overline{\mathbf{f}})$ ou $(\mathbf{F})$.

Como veremos, no caso do sistema (1.1), a condição de não ressonância é $a \pm b \notin$ $\sigma(-\Delta)$. Nosso objetivo é garantir a existência de pelo menos duas soluções quando os 
valores $a+b$ ou $a-b$ estão perto de um autovalor do operador Laplaciano. Primeiramente obteremos um resultado no caso em que um dos valores $a \pm b$ está perto da ressonância, enquanto o outro está a uma certa distância do espectro $\sigma(-\Delta)$ (veja os Teoremas 3.1 e 3.2). Em seguida consideramos o caso em que ambos os valores estão perto da ressonância (veja os Teoremas 3.3 e 3.4).

O Teorema a seguir garante a existência de duas soluções distintas quando $a+b$ é quase ressonante, enquanto $a-b$ está distante do espectro $\sigma(-\Delta)$.

Teorema 3.1. Sejam $\lambda_{k}$ um autovalor de $(-\Delta), \lambda_{l}$ o primeiro autovalor acima de $a-b$ $e$

$$
Z=\operatorname{span}\left\{(\phi, \phi): \phi \in H_{\lambda_{k}}\right\}
$$

Suponhamos $h_{1}, h_{2} \in L^{2}(\Omega)$, e que $f_{1}, f_{2}$ satisfaçam as hipóteses (1.2) e (ff) (ou (E)). Então

(a) Dado $\delta>0$, existe $\varepsilon_{0}>0$ tal que, se dist $(a-b, \sigma(-\Delta))>\delta$ e $a+b \in\left(\lambda_{k}-\varepsilon_{0}, \lambda_{k}\right)$, então o problema $(1.1+)$ possui duas soluções distintas.

(b) Dado $\delta>0$, existe $\varepsilon_{1}>0$ tal que, se dist $(a-b, \sigma(-\Delta))>\delta$ e $a+b \in\left(\lambda_{k}, \lambda_{k}+\varepsilon_{1}\right)$, então o problema (1.1-) possui duas soluções distintas.

Como consequência do Teorema [3.1, é possível mostrar também um resultado de existência de soluções para o caso em que $a-b$ é quase ressonante, enquanto $a+b$ está distante de $\sigma(-\Delta)$.

Teorema 3.2. Sejam $\lambda_{k}$ um autovalor de $(-\Delta), \lambda_{l}$ o primeiro autovalor acima de $a+b$ $e$

$$
Z=\operatorname{span}\left\{(\phi,-\phi): \phi \in H_{\lambda_{k}}\right\}
$$

Suponhamos $h_{1}, h_{2} \in L^{2}(\Omega)$, e que $f_{1}, f_{2}$ satisfaçam as hipóteses (1.2) e (f) (ou $(\boldsymbol{F})$ ). Então

(c) Dado $\delta>0$, existe $\varepsilon_{0}>0$ tal que, se dist $(a+b, \sigma(-\Delta))>\delta$ e $a-b \in\left(\lambda_{k}-\varepsilon_{0}, \lambda_{k}\right)$, então o problema (1.1-) possui duas soluções distintas.

(d) Dado $\delta>0$, existe $\varepsilon_{1}>0$ tal que, se dist $(a+b, \sigma(-\Delta))>\delta$ e $a-b \in\left(\lambda_{k}, \lambda_{k}+\varepsilon_{1}\right)$, então o problema $(1.1+)$ possui duas soluções distintas. 
Comparando o item (a) do Teorema 3.1 com o item (d) do Teorema 3.2, observemos que o problema $(1.1+)$ possui duas soluções tanto quando $a+b$ é quase ressonante inferiormente ao autovalor, como quando $a-b$ é quase ressonante superiormente ao autovalor. No resultado a seguir, mostramos a existência de duas soluções também quando estas duas quase ressonâncias acontecem simultaneamente.

Teorema 3.3. Sejam $\lambda_{k}$ e $\lambda_{l}$ dois autovalores (não necessariamente distintos) de $(-\Delta)$, $e$

$$
Z=\operatorname{span}\left\{(\phi, \phi): \phi \in H_{\lambda_{k}}, \quad(\phi,-\phi): \phi \in H_{\lambda_{l}}\right\}
$$

Suponhamos $h_{1}, h_{2} \in L^{2}(\Omega)$, e que $f_{1}, f_{2}$ satisfaçam as hipóteses (1.2) e (f) (ou (ㅍ) ). Então

(e) existe $\varepsilon_{2}>0$ tal que, se $a-b \in\left(\lambda_{l}, \lambda_{l}+\varepsilon_{2}\right)$ e $a+b \in\left(\lambda_{k}-\varepsilon_{2}, \lambda_{k}\right)$, então o problema $(1.1+)$ possui duas soluções distintas.

O caso do problema (1.1-) quando, simultaneamente, $a+b$ é quase ressonante superiormente ao autovalor e $a-b$ é quase ressonante inferiormente ao autovalor, segue de novo facilmente do Teorema 3.3 .

Teorema 3.4. Sejam $\lambda_{k}$ e $\lambda_{l}$ dois autovalores (não necessariamente distintos) de $(-\Delta)$, $e$

$$
Z=\operatorname{span}\left\{(\phi,-\phi): \phi \in H_{\lambda_{k}}, \quad(\phi, \phi): \phi \in H_{\lambda_{l}}\right\}
$$

Suponhamos $h_{1}, h_{2} \in L^{2}(\Omega)$, e que $f_{1}, f_{2}$ satisfaçam as hipóteses (1.2) e (f) (ou (ㅍ) ). Então

(f) Existe $\varepsilon_{2}>0$ tal que, se $a-b \in\left(\lambda_{k}-\varepsilon_{2}, \lambda_{k}\right)$ e $a+b \in\left(\lambda_{l}, \lambda_{l}+\varepsilon_{2}\right)$, então o problema (1.1-) possui duas soluções distintas.

Juntando os Teoremas de 3.1 a 3.4, é possível esboçar um gráfico qualitativo das regiões, no plano $(a+b, a-b)$, onde podemos garantir a existência de pelo menos duas soluções para o problema (1.1-): veja Figura [3.1, O gráfico para o problema $(1.1+)$ é análogo, com os eixos trocados.

Observação 3.5. A hipótese $(\underline{\mathbf{f}})$ é mais forte que $(\underline{\mathbf{F}}-i)$, mas ela não exige a condição adicional sobre $h_{1}, h_{2}$ contida em (F-ii). Por esta razão consideramos ambos conjuntos de hipóteses. Observemos que estas hipóteses lidam apenas com o comportamento assintótico 
Figura 3.1: Esboço das regiões de duas soluções para problema (1.1-)

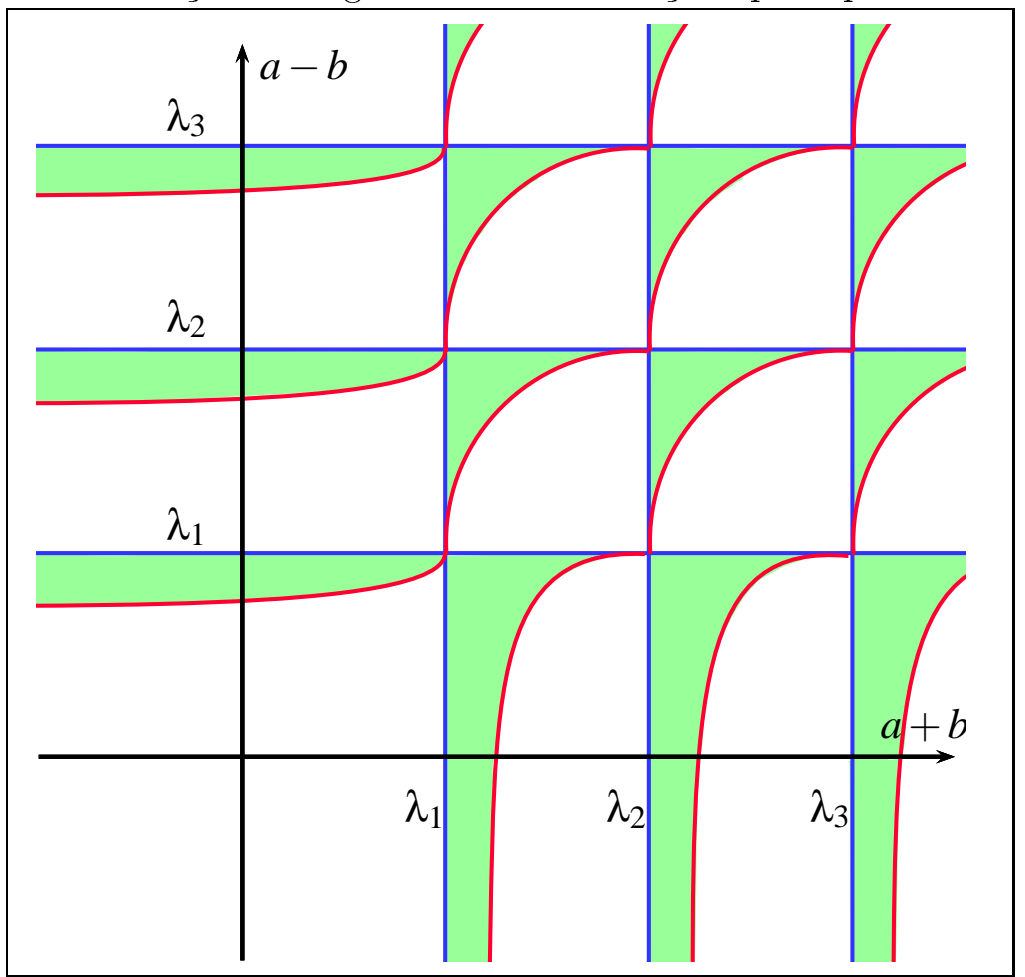

de $f_{1}, f_{2}$, uma vez que nenhuma condição na origem é exigida para nossos resultados de multiplicidade.

Exemplos de funções que satisfazem $(\underline{\mathbf{f}})$, são

$$
f(x, u)=|u|^{q-2} u, \quad \text { ou } \quad f(x, u)=\ln (1+|u|) \arctan (u) .
$$

Para o caso $(\mathbf{F})$, podemos considerar por exemplo,

$$
f(x, u)=\arctan (u) \quad \text { ou } \quad f(x, u)= \begin{cases}u & \text { se }|u| \leq 1 \\ \frac{1}{u} & \text { se }|u|>1\end{cases}
$$

Notemos que este último exemplo ainda satisfaz as hipóteses utilizadas em [KT11a, KT11b, mas para nenhuma escolha das funções $h_{1}, h_{2}$ (nem mesmo para $h_{1}=h_{2}=0$ ), a condição do tipo Landesman-Lazer que eles assumem estaria satisfeita.

Observação 3.6. Observemos que seria possível pensar em estudar um sistema mais 
geral que (1.1), cuja parte linear dependa de quatro parâmetros independentes:

$$
\begin{cases}-\Delta u=a u+c v \pm\left(f_{1}(x, v)+h_{1}(x)\right) & \text { em } \Omega \\ -\Delta v=d u+e v \pm\left(f_{2}(x, u)+h_{2}(x)\right) & \text { em } \Omega \\ u(x)=v(x)=0 & \text { em } \partial \Omega .\end{cases}
$$

O sistema (3.1) pode ser reescrito na forma vetorial

$$
\begin{cases}-\Delta\left[\begin{array}{l}
u \\
v
\end{array}\right]=A\left[\begin{array}{l}
u \\
v
\end{array}\right] \pm\left[\begin{array}{l}
f_{1}(x, v)+h_{1}(x) \\
f_{2}(x, u)+h_{2}(x)
\end{array}\right] \text { em } \Omega \\
u(x)=v(x)=0 & \text { em } \partial \Omega\end{cases}
$$

onde $A=\left(\begin{array}{ll}a & c \\ d & e\end{array}\right)$, de autovalores $\nu_{1,2}=\frac{a+e}{2} \pm \sqrt{\left(\frac{a-e}{2}\right)^{2}+c d}$

Como pretendemos usar métodos variacionais para obter nossos resultados, precisamos considerar $a=e$ na matriz $A$, e pedir que ela tenha autovalores reais, isto é, impor $c d \geq 0$. A forma do sistema (3.1) pode ser simplificada ainda mais graças ao seguinte lema.

Lema 3.7 (Lema 3.1 de [Mas07]). Se $(U, V)$ é solução de

$$
\begin{cases}-\Delta U=a U+c V / \lambda \pm\left(f_{1}(x, V)+h_{1}\right) / \lambda & \text { em } \Omega \\ -\Delta V=d \lambda U+e V \pm\left(f_{2}(x, \lambda U)+h_{2}\right) & \text { em } \Omega \\ U(x)=V(x)=0 & \text { em } \partial \Omega\end{cases}
$$

para algum $\lambda>0$, então $(u, v)=(\lambda U, V)$ é uma solução de (3.1).

Demonstração. Seja $(U, V) \in E$ solução de (3.3) para algum $\lambda>0$. Assim vale

$$
\begin{cases}-\Delta \lambda U=a \lambda U+c V \pm\left(f_{1}(x, V)+h_{1}\right) & \text { em } \Omega \\ -\Delta V=d \lambda U+e V \pm\left(f_{2}(x, \lambda U)+h_{2}\right) & \text { em } \Omega \\ \lambda U(x)=V(x)=0 & \text { em } \partial \Omega\end{cases}
$$

mostrando que $(u, v)=(\lambda U, V)$ é solução de (3.1) .

De fato, se ainda supormos que $c d \neq 0$, então tomando $\lambda=\sqrt{c / d}$ no sistema (3.3), 
segue que os dois termos da diagonal secundária da matriz $A$ tornam-se iguais, enquanto as novas funções $f_{1}(x, V) / \lambda, h_{1} / \lambda$ e $f_{2}(x, \lambda U)$ ainda satisfazem todas as hipóteses satisfeitas por $f_{i}, h_{i}, i=1,2$. Portanto, se $a=e$ e $c d>0$, podemos transformar o sistema (3.1) no sistema (1.1), onde $b=\sqrt{c d}$. Neste caso os autovalores de $A$ são $a \pm b$.

Observemos que no caso $c=0$ ou $d=0$ os dois autovalores de $A$ coincidem, o que torna impossível satisfazer as hipóteses dos Teoremas de 3.1 a 3.4 .

Este capítulo possui a seguinte estrutura: na Seção 3.1 estabelecemos algumas notações e mostramos alguns lemas preliminares; na Seção 3.2 demonstramos os Teoremas $3.1 \mathrm{e} 3.2$. na Seção 3.3 demonstramos os Teoremas 3.3 e 3.4, e por fim, na Seção 3.4, demonstramos as condições de tipo (PS) que precisamos.

\subsection{Notações e resultados preliminares}

Ao longo deste capítulo denotamos, $E=H_{0}^{1}(\Omega) \times H_{0}^{1}(\Omega)$. Se $u, v \in H_{0}^{1}(\Omega)$ e $\mathbf{u}=$ $(u, v) \in E$, utilizamos as seguintes normas

$$
\begin{cases}\|u\|_{L^{2}}=\sqrt{\int_{\Omega} u^{2}}, & \|\mathbf{u}\|_{\left[L^{2}\right]^{2}}=\sqrt{\|u\|_{L^{2}}^{2}+\|v\|_{L^{2}}^{2}}, \\ \|u\|_{H}=\sqrt{\int_{\Omega}|\nabla u|^{2}}, & \|\mathbf{u}\|_{E}=\sqrt{\|u\|_{H}^{2}+\|v\|_{H}^{2}} .\end{cases}
$$

Os produtos internos em $L^{2}(\Omega) \times L^{2}(\Omega)$ e em $E$, associados às normas acima, denotamos por $\langle\cdot, \cdot\rangle_{\left[L^{2}\right]^{2}}$ e $\langle\cdot, \cdot\rangle_{E}$, respectivamente. Observemos que pela Desigualdade de Poincaré (ver Teorema A.12), temos $\|u\|_{L^{2}} \leq S\|u\|_{H}$ para alguma constante positiva $S$; sem perda de generalidade vamos assumir ao longo desde capítulo $S=1$, a fim de simplificar as estimativas.

Definamos as aplicações $\mathcal{F}: E \rightarrow \mathbb{R}$ e $\mathcal{H}: E \rightarrow \mathbb{R}$, dadas por

$$
\mathcal{F}(u, v)=\int_{\Omega} F_{1}(x, v)+\int_{\Omega} F_{2}(x, u), \quad \mathcal{H}(u, v)=\int_{\Omega} h_{1} v+\int_{\Omega} h_{2} u
$$

Além disso, definamos a forma bilinear $B_{a, b}: E \times E \rightarrow \mathbb{R}$ :

$$
B_{a, b}((u, v),(\phi, \psi))=\int_{\Omega} \nabla u \nabla \psi+\int_{\Omega} \nabla v \nabla \phi-a \int_{\Omega}(u \psi+v \phi)-b \int_{\Omega}(u \phi+v \psi) .
$$


A fim de simplificar a notação, estaremos denotando a forma $B_{a, b}$ por $B$, utilizando $B_{a, b}$ apenas quando for necessário evidenciar a dependência dos valores $a, b$.

Os funcionais associados aos problemas (1.1 1 ), são $J_{a, b}^{ \pm}: E \rightarrow \mathbb{R}$, dados por

$$
J_{a, b}^{ \pm}(u, v)= \pm \frac{1}{2} B_{a, b}((u, v),(u, v))-\mathcal{F}(u, v)-\mathcal{H}(u, v)
$$

Do mesmo modo que para a forma bilinear $B$, denotaremos estes funcionais apenas por $J^{ \pm}$, utilizando $J_{a, b}^{ \pm}$apenas quando for necessário evidenciar a dependência dos valores $a, b$. Notemos que este funcional é de classe $\mathcal{C}^{1}$ (ver Apêndice C).

Denotando $\mathbf{u}=(u, v), \boldsymbol{\phi}=(\phi, \psi)$ e $\overline{\boldsymbol{\phi}}=(\psi, \phi)$, observamos que

$$
\begin{gathered}
B(\mathbf{u}, \boldsymbol{\phi})=\langle\mathbf{u}, \overline{\boldsymbol{\phi}}\rangle_{E}-a\langle\mathbf{u}, \overline{\boldsymbol{\phi}}\rangle_{\left[L^{2}\right]^{2}}-b\langle\mathbf{u}, \boldsymbol{\phi}\rangle_{\left[L^{2}\right]^{2}}, \\
J^{ \pm}(\mathbf{u})= \pm \frac{1}{2} B(\mathbf{u}, \mathbf{u})-(\mathcal{F}(\mathbf{u})+\mathcal{H}(\mathbf{u})) \\
\left(J^{ \pm}\right)^{\prime}(\mathbf{u})[\boldsymbol{\phi}]= \pm B(\mathbf{u}, \boldsymbol{\phi})-\left(\mathcal{F}^{\prime}(\mathbf{u})[\boldsymbol{\phi}]+\mathcal{H}^{\prime}(\mathbf{u})[\boldsymbol{\phi}]\right) .
\end{gathered}
$$

Nos próximos lemas apresentamos algumas estimativas que são utilizadas ao longo deste capítulo.

Denotaremos (neste e nos demais capítulos da tese) por $C, C_{0}, C_{1}, C_{2}, \ldots$ constantes positivas, das quais o valor não é relevante e que podem assumir valores diferentes de uma linha para outra.

Lema 3.8. Sejam $f_{1}, f_{2}$ satisfazendo (1.2) e $h_{1}, h_{2} \in L^{2}(\Omega)$. Então existem constantes $S_{0}>0$ e $H>0$, tais que

$$
\begin{aligned}
|\mathcal{H}(\mathbf{u})| \leq H\|\mathbf{u}\|_{\left[L^{2}\right]^{2}}, & \left|\mathcal{H}^{\prime}(\mathbf{u})[\boldsymbol{\phi}]\right| \leq H\|\boldsymbol{\phi}\|_{\left[L^{2}\right]^{2}} \\
|\mathcal{F}(\mathbf{u})| \leq S_{0}\left(1+\|\mathbf{u}\|_{\left[L^{2}\right]^{2}}^{q}\right), & \left|\mathcal{F}^{\prime}(\mathbf{u})[\boldsymbol{\phi}]\right| \leq S_{0}\left(1+\|\mathbf{u}\|_{\left[L^{2}\right]^{2}}^{q-1}\right)\|\boldsymbol{\phi}\|_{\left[L^{2}\right]^{2}},
\end{aligned}
$$

para todo $\mathbf{u}=(u, v) \in E$ e $\boldsymbol{\phi}=(\phi, \psi) \in E$.

Demonstração. Sejam $(u, v),(\phi, \psi) \in E$. Pela Desigualdade de Hölder (Teorema A.7), temos

$$
\left|\int_{\Omega} h_{1} v\right| \leq\left\|h_{1}\right\|_{L^{2}}\|v\|_{L^{2}} \text { and }\left|\int_{\Omega} h_{2} u\right| \leq\left\|h_{2}\right\|_{L^{2}}\|u\|_{L^{2}} .
$$


Então existe $H>0$, dependendo de $h_{1}$ e $h_{2}$, tal que

$$
|\mathcal{H}(\mathbf{u})| \leq \frac{H}{2}\|u\|_{L^{2}}+\frac{H}{2}\|v\|_{L^{2}} \leq H\|\mathbf{u}\|_{\left[L^{2}\right]^{2}}
$$

A segunda estimativa em (3.9) $)$ segue diretamente, uma vez que $\mathcal{H}^{\prime}(\mathbf{u})[\boldsymbol{\phi}]=\mathcal{H}(\boldsymbol{\phi})$.

Para mostrar ( $(3.10)$, notemos que por (1.2), temos

$$
\left|\int_{\Omega} F_{1}(x, v)\right| \leq \int_{\Omega} S\left(|v|+\frac{|v|^{q}}{q}\right)=S\|v\|_{L^{1}}+\frac{S}{q}\|v\|_{L^{q}}^{q}
$$

e pela Desigualdade de Hölder, segue

$$
\|v\|_{q}^{q}=\int_{\Omega}|v|^{q} \leq\left(\int_{\Omega}|v|^{q \frac{2}{q}}\right)^{\frac{q}{2}}|\Omega|^{\frac{2-q}{q}}=\|v\|_{L^{2}}^{q}|\Omega|^{\frac{2-q}{q}}
$$

enquanto que pela imersão contínua $L^{2}(\Omega) \hookrightarrow L^{1}(\Omega)$, temos $\|v\|_{L^{1}} \leq C\|v\|_{L^{2}}$, para uma constante $C>0$, dependendo de $\Omega$. Logo,

$$
\left|\int_{\Omega} F_{1}(x, v)\right| \leq S C\|v\|_{L^{2}}+\frac{S}{q}|\Omega|^{\frac{2-q}{q}}\|v\|_{L^{2}}^{q} \leq\left(S C+\frac{S}{q}|\Omega|^{\frac{2-q}{q}}\right)\left(1+\|v\|_{L^{2}}^{q}\right)
$$

e vale estimativa análoga para $\int_{\Omega} F_{2}(x, u)$. Então, tomando $\frac{\widetilde{S}_{0}}{2}=\left(S C+\frac{S}{q}|\Omega|^{\frac{2-q}{q}}\right)$, temos

$$
|\mathcal{F}(\mathbf{u})| \leq\left|\int_{\Omega} F_{1}(x, v)\right|+\left|\int_{\Omega} F_{2}(x, u)\right| \leq \frac{\widetilde{S}_{0}}{2}\left(1+\|v\|_{L^{2}}^{q}\right)+\frac{\widetilde{S}_{0}}{2}\left(1+\|u\|_{L^{2}}^{q}\right) \leq \widetilde{S}_{0}\left(1+\|\mathbf{u}\|_{\left[L^{2}\right]^{2}}^{q}\right),
$$

onde usamos $\|u\|_{L^{2}}^{r}+\|v\|_{L^{2}}^{r} \leq 2\|u\|_{L^{2}}^{r} \leq 2\|\mathbf{u}\|_{\left[L^{2}\right]^{2}}^{r}$.

Por outro lado,

$$
\left|\mathcal{F}^{\prime}(\mathbf{u})[\phi]\right|=\left|\int_{\Omega} f_{1}(x, v) \psi+f_{2}(x, u) \phi\right| \leq\left|\int_{\Omega} f_{1}(x, v) \psi\right|+\left|\int_{\Omega} f_{2}(x, u) \phi\right|
$$

onde, por (1.2), $\left|\int_{\Omega} f_{1}(x, v) \psi\right| \leq \int_{\Omega} S\left(1+|v|^{q-1}\right)|\psi|$. Pela Desigualdade de Hölder com $r=\frac{2}{3-q}<2$, temos

$$
\begin{aligned}
\left|\int_{\Omega} f_{1}(x, v) \psi\right| & \leq C S\left(\|\psi\|_{L^{1}}+\left(\int_{\Omega}|v|^{q-1 \frac{2}{q-1}}\right)^{\frac{q-1}{2}}\|\psi\|_{L^{r}}\right) \\
& \leq S C_{1}\left(\|\psi\|_{L^{2}}+\|v\|_{L^{2}}^{q-1}\|\psi\|_{L^{2}}\right) .
\end{aligned}
$$


Novamente, temos estimativa análoga para $\left|\int_{\Omega} f_{2}(x, u) \phi\right|$ e portanto, tomando $\frac{\widehat{S}_{0}}{2}=S C_{1}$, temos

$$
\left|F^{\prime}(\mathbf{u})[\boldsymbol{\phi}]\right| \leq \frac{\widehat{S}_{0}}{2}\left(1+\|v\|_{L^{2}}^{q-1}\right)\|\psi\|_{L^{2}}+\frac{\widehat{S}_{0}}{2}\left(1+\|u\|_{L^{2}}^{q-1}\right)\|\phi\|_{L^{2}} \leq \widehat{S}_{0}\left(1+\|\mathbf{u}\|_{\left[L^{2}\right]^{2}}^{q-1}\right)\|\phi\|_{\left[L^{2}\right]^{2}}
$$

Basta agora tomarmos $S_{0}=\max \left\{\widetilde{S}_{0}, \widehat{S}_{0}\right\}$

Outro resultado que precisamos é o seguinte lema que trata da continuidade dos operadores de Nemytskii associados a $f_{1}$ e $f_{2}$ (ver Apêndice B).

Lema 3.9. Se $f_{1}, f_{2}$ satisfazem (1.2), então $N_{f_{1}}, N_{f_{2}}: L^{2}(\Omega) \rightarrow L^{2}(\Omega)$, estão bem definidos e são contínuos, onde $N_{f_{1}}$ e $N_{f_{2}}$ denotam os operadores de Nemytskii associados a $f_{1}$ e $f_{2}$, respectivamente.

Demonstração. Primeiramente mostremos que $f_{i}(\cdot, u(\cdot)) \in L^{2}(\Omega)$ sempre que $u \in L^{2}(\Omega)$, para $i=1,2$. De fato, se $u \in L^{2}(\Omega)$, então temos

$$
\int_{\Omega}\left|f_{i}(x, u(x))\right|^{2} \leq S^{2} \int_{\Omega}\left(1+|u|^{q-1}\right)^{2} \leq S^{2} \int_{\Omega} C\left(1+u^{2}\right)
$$

onde a última desigualdade é consequência do fato que a função $t \mapsto \frac{\left(1+|t|^{q-1}\right)^{2}}{1+t^{2}}$ é positiva, contínua em $\mathbb{R}$ e tem limite no infinito igual a zero, logo é limitada. Obtemos então

$$
\int_{\Omega}\left|f_{i}(x, u(x))\right|^{2} \leq S^{2} C|\Omega|+S^{2} C\|u\|_{L^{2}}^{2}<\infty
$$

uma vez que $\Omega$ é limitado. Isto mostra que os operadores $N_{f_{1}}$ e $N_{f_{2}}$ estão bem definidos e sua continuidade segue do Teorema B.3.

A fim de estudar os funcionais $J^{ \pm}$, definamos uma base ortogonal para $E$ que diagonalize $B$, como feito em [Mas07]. Para isso, consideremos o problema de autovalores: $(u, v) \in E$

$$
B((u, v),(\phi, \psi))=\mu\langle(u, v),(\phi, \psi)\rangle_{E}, \quad \forall(\phi, \psi) \in E .
$$

Para qualquer $i \in \mathbb{N}$, pondo $(\phi, \psi)=\left(0, \phi_{i}\right)$ em (3.18), temos

$$
\int_{\Omega} \nabla u \nabla \phi_{i}-a \int_{\Omega} u \phi_{i}-b \int_{\Omega} v \phi_{i}=\mu \int_{\Omega} \nabla v \nabla \phi_{i}
$$


integrando por partes e usando $-\Delta \phi_{i}=\lambda_{i} \phi_{i}$, obtemos

$$
a \int_{\Omega} u \phi_{i}-\lambda_{i} \int_{\Omega} u \phi_{i}+\mu \lambda_{i} \int_{\Omega} v \phi_{i}+b \int_{\Omega} v \phi_{i}=0
$$

Analogamente, pondo $(\phi, \psi)=\left(\phi_{i}, 0\right)$ em (3.18) $)$, temos

$$
\mu \lambda_{i} \int_{\Omega} u \phi_{i}+b \int_{\Omega} u \phi_{i}+a \int_{\Omega} v \phi_{i}-\lambda_{i} \int_{\Omega} v \phi_{i}=0
$$

Sejam $u=\sum_{j \in \mathbb{N}} c_{j} \phi_{j}$ e $v=\sum_{j \in \mathbb{N}} d_{j} \phi_{j}$. Como as autofunções $\phi_{i}$ são duas a duas ortogonais, segue de (3.20) e (3.21)

$$
\left\{\begin{array}{l}
a c_{i}-\lambda_{i} c_{i}+\mu \lambda_{i} d_{i}+b d_{i}=0, \\
\mu \lambda_{i} c_{i}+b c_{i}+a d_{i}-\lambda_{i} d_{i}=0 .
\end{array}\right.
$$

Logo (3.18) é equivalente a

$$
\left(\begin{array}{cc}
a-\lambda_{i} & \mu \lambda_{i}+b \\
\mu \lambda_{i}+b & a-\lambda_{i}
\end{array}\right)\left(\begin{array}{l}
c_{i} \\
d_{i}
\end{array}\right)=0, \quad \forall i \in \mathbb{N},
$$

que possui soluções não triviais quando $\mu$ é tal que o determinante da matriz acima é nulo para algum $i \in \mathbb{N}$, isto é, $\left(a-\lambda_{i}\right)^{2}-\left(\mu \lambda_{i}+b\right)^{2}=0$. Portanto definamos as duas sequências de autovalores

$$
\mu_{ \pm i}=\frac{-b \pm\left(\lambda_{i}-a\right)}{\lambda_{i}}, i \in \mathbb{N}
$$

e as autofunções correspondentes

$$
\boldsymbol{\psi}_{ \pm i}=\frac{\left(\phi_{i}, \pm \phi_{i}\right)}{\sqrt{2 \lambda_{i}}}, i \in \mathbb{N}
$$

Desta forma, temos para todo $i, j \in \mathbb{Z}_{0}=\mathbb{Z} \backslash\{0\}$

$$
\left\|\boldsymbol{\psi}_{i}\right\|_{E}=1, \quad\left\langle\boldsymbol{\psi}_{i}, \boldsymbol{\psi}_{j}\right\rangle_{E}=\delta_{i, j}, \quad B\left(\boldsymbol{\psi}_{i}, \boldsymbol{\psi}_{j}\right)=\mu_{i} \delta_{i, j}, \quad\left\langle\boldsymbol{\psi}_{i}, \boldsymbol{\psi}_{j}\right\rangle_{\left[L^{2}\right]^{2}}=\lambda_{|i|}^{-1} \delta_{i, j}
$$


Como consequência, se $\mathbf{u}=\sum_{i \in \mathbb{Z}_{0}} c_{i} \boldsymbol{\psi}_{i}$, temos

$$
\|\mathbf{u}\|_{E}^{2}=\sum_{i \in \mathbb{Z}_{0}} c_{i}^{2}, \quad B(\mathbf{u}, \mathbf{u})=\sum_{i \in \mathbb{Z}_{0}} \mu_{i} c_{i}^{2}, \quad\|\mathbf{u}\|_{\left[L^{2}\right]^{2}}^{2}=\sum_{i \in \mathbb{Z}_{0}} \lambda_{|i|}^{-1} c_{i}^{2}
$$

\subsection{Demonstração dos principais resultados}

Nesta seção demonstramos os principais teoremas, em particular, apresentamos a prova do item (a) do Teorema 3.1. Encontramos duas geometrias de sela na Seção 3.2.1, das quais obtemos (na Seção 3.2.2) duas sequências de pontos críticos das aproximações finito dimensionais do funcional $J^{+}$e mostramos, na Seção 3.2.4, como obter duas soluções distintas do problema (1.1) a partir destas sequências. Na Seção 3.2 .3 explicamos o que dever ser adaptado para o item (b) do Teorema 3.1, enquanto o Teorema 3.2 é demonstrado no final da Seção 3.2.4.

\subsubsection{Duas geometrias de ponto de sela quando $a+b$ é quase res- sonante inferiormente}

Nesta seção encontramos as duas geometrias de sela, que nos permitirão encontrar duas soluções para o problema $(\underline{1.1}+)$ quando $a+b$ é suficientemente próximo de $\lambda_{k}$ por baixo e $a-b$ longe de $\sigma(-\Delta)$.

Fixemos $\lambda_{k}$ (de multiplicidade $m$, em particular seja $\lambda_{k}=\lambda_{k+m-1}$ ) e $\lambda_{l}$ os autovalores anunciados no Teorema 3.1. Definamos os subespaços de $E$

$$
\left\{\begin{array}{l}
V=\overline{\operatorname{span}\left\{\boldsymbol{\psi}_{i}: i \in \mathbb{Z}_{0}, \mu_{i}<0 \text { e } \mu_{i} \neq \mu_{k}\right\}}, \\
Z=\overline{\operatorname{span}\left\{\boldsymbol{\psi}_{i}: i \in \mathbb{Z}_{0}, \mu_{i}=\mu_{k}\right\}}, \\
W=\overline{\operatorname{span}\left\{\boldsymbol{\psi}_{i}: i \in \mathbb{Z}_{0}, \mu_{i}>0 \text { e } \mu_{i} \neq \mu_{k}\right\}}
\end{array}\right.
$$

e denotemos por $B_{V}, B_{V Z}, B_{W}$ e $B_{Z W}$ as bolas unitárias fechadas, com respeito à norma $\|\cdot\|_{E}$, nos espaços $V, V \oplus Z, W$ e $Z \oplus W$ respectivamente, e por $S_{V}, S_{V Z}, S_{W}$ e $S_{Z W}$ suas respectivas fronteiras relativas. 
Definamos também os conjuntos de índices

$$
\begin{gathered}
\mathbb{Z}_{V}=\left\{i \in \mathbb{Z}_{0}: \mu_{i}<0 \text { e } \mu_{i} \neq \mu_{k}\right\}, \\
\mathbb{Z}_{Z}=\left\{i \in Z_{0}: \mu_{i}=\mu_{k}\right\}, \\
\mathbb{Z}_{W}=\left\{i \in \mathbb{Z}_{0}: \mu_{i}>0 \text { e } \mu_{i} \neq \mu_{k}\right\} .
\end{gathered}
$$

Observemos que se $a+b \in\left(\lambda_{k}-\varepsilon, \lambda_{k}\right)$ para algum $\varepsilon>0$, então

$$
0<\mu_{k}=1-\frac{a+b}{\lambda_{k}}=\frac{\lambda_{k}-(a+b)}{\lambda_{k}}<\frac{\varepsilon}{\lambda_{k}}
$$

Começamos com um lema que obtém estimativas nos subespaços $V, Z$ e $W$.

Lema 3.10. Suponhamos $a \pm b \notin \sigma(-\Delta)$ e fixemos $\lambda_{k}$ o primeiro autovalor maior que $a+b$, de multiplicidade $m$, e $\lambda_{l}$ o primeiro autovalor maior que $a-b$.

Se dist $(a-b, \sigma(-\Delta))>\delta>0$, então existe uma constante $K_{a+b, \delta}>0$, dependendo da soma $a+b$ e de $\delta$, tal que

$$
\begin{array}{ll}
B(\mathbf{u}, \mathbf{u}) \leq-K_{a+b, \delta}\|\mathbf{u}\|_{E}^{2}, & \forall \mathbf{u} \in V, \\
B(\mathbf{u}, \mathbf{u}) \geq K_{a+b, \delta}\|\mathbf{u}\|_{E}^{2}, & \forall \mathbf{u} \in Z \oplus W .
\end{array}
$$

Além disso, se $a+b$ está suficientemente perto de $\lambda_{k}$ (em particular, se $a+b>0$ e $\left.\operatorname{dist}\left(a+b, \sigma(-\Delta) \backslash\left\{\lambda_{k}\right\}\right)>\alpha>0\right)$, então existe $G_{\alpha, \delta}>0$, dependendo de $\alpha$ e $\delta$, tal que

$$
\begin{array}{cl}
B(\mathbf{u}, \mathbf{u}) \leq-G_{\alpha, \delta}\|\mathbf{u}\|_{E}^{2}, & \forall \mathbf{u} \in V \\
B(\mathbf{u}, \mathbf{u}) \geq G_{\alpha, \delta}\|\mathbf{u}\|_{E}^{2}, & \forall \mathbf{u} \in W .
\end{array}
$$

Observação 3.11. Como pode ser visto na demonstração abaixo, as constantes $K_{a+b, \delta} e$ $G_{\alpha, \delta}$ dependem também de $k$ e de $l$, que estamos considerando fixadas.

Demonstração do Lema 3.10. Por hipótese $\lambda_{k}$ é o primeiro autovalor maior que $a+b$, então temos

$$
a+b \in\left(\lambda_{k-1}, \lambda_{k}\right), \text { se } k \geq 2 \quad \text { ou } \quad a+b<\lambda_{1} \text {. }
$$

Caso $k \geq 2$, a sequência $\left\{\mu_{i}=1-\frac{a+b}{\lambda_{i}}\right\}_{i \in \mathbb{N}}$ é crescente, com $\mu_{k-1}<0$ e $\mu_{k}>0$. Assim 
vale

$$
\min _{i \in \mathbb{N}}\left|\mu_{i}\right|=\min \left\{\mu_{k},-\mu_{k-1}\right\}=\min \left\{1-\frac{a+b}{\lambda_{k}}, \frac{a+b}{\lambda_{k-1}}-1\right\} .
$$

Se $a+b<\lambda_{1}$, vale

$$
\inf _{i \in \mathbb{N}}\left|\mu_{i}\right| \geq \min \left\{1,1-\frac{a+b}{\lambda_{1}}\right\}
$$

Portanto, em ambos os casos, existe uma constante $K_{a+b}>0$, dependendo da soma $a+b($ e de $k)$, tal que

$$
\left|\mu_{i}\right| \geq K_{a+b} \text {, para todo } i \in \mathbb{N} \text {. }
$$

Do mesmo modo, como $\lambda_{l}$ é o primeiro autovalor maior que $a-b$ e $\operatorname{dist}(a-b, \sigma(-\Delta))>$ $\delta>0$, temos

$$
a-b \in\left(\lambda_{l-1}+\delta, \lambda_{l}-\delta\right), \text { se } l \geq 2 \quad \text { ou } \quad a-b<\lambda_{1}-\delta .
$$

Caso $l \geq 2$, a sequência $\left\{\mu_{-i}=-1+\frac{a-b}{\lambda_{i}}\right\}_{i \in \mathbb{N}}$ é decrescente, com $\mu_{-(l-1)}>0$ e $\mu_{-l}<0$. Assim

$$
\begin{aligned}
\min _{i \in \mathbb{N}}\left|\mu_{-i}\right| & =\min \left\{\mu_{-(l-1)},-\mu_{-l}\right\}=\min \left\{-1+\frac{a-b}{\lambda_{l-1}},-\frac{a-b}{\lambda_{l}}+1\right\} \\
& \geq \min \left\{\frac{\delta}{\lambda_{l-1}}, \frac{\delta}{\lambda_{l}}\right\}=\frac{\delta}{\lambda_{l}} .
\end{aligned}
$$

Se $a-b<\lambda_{1}-\delta$, temos

$$
\inf _{i \in \mathbb{N}}\left|\mu_{-i}\right| \geq \min \left\{1,1-\frac{a-b}{\lambda_{1}}\right\} \geq \min \left\{1, \frac{\delta}{\lambda_{1}}\right\}
$$

Portanto, em ambos os casos, existe uma constante $K_{\delta}>0$ dependendo de $\delta$ (e de $l$ ), tal que

$$
\left|\mu_{-i}\right| \geq K_{\delta} \text {, para todo } i \in \mathbb{N} \text {. }
$$

Concluímos assim, por (3.33) e (3.35) que existe constante $K_{a+b, \delta}>0$, dependendo da soma $a+b$ e de $\delta$, tal que

$$
\left|\mu_{ \pm i}\right| \geq K_{a+b, \delta}, \text { para todo } i \in \mathbb{N} \text {. }
$$

Logo, se $\mathbf{u} \in V$, podemos escrever $\mathbf{u}=\sum_{j \in \mathbb{Z}_{V}} c_{j} \boldsymbol{\psi}_{j}$, onde $\mu_{j} \leq-K_{a+b, \delta}$ para $j \in \mathbb{Z}_{V} \mathrm{e}$ 
$\operatorname{logo} B(\mathbf{u}, \mathbf{u})=\sum_{j \in \mathbb{Z}_{V}} \mu_{j} c_{j}^{2} \leq-K_{a+b, \delta}\|\mathbf{u}\|_{E}^{2}$, provando (3.28).

Analogamente, se $\mathbf{u}=\sum_{j \in \mathbb{Z}_{Z} \cup \mathbb{Z}_{W}} c_{j} \psi_{j} \in Z \oplus W$, temos $\mu_{j} \geq K_{a+b, \delta}$ para todo $j \in$ $\mathbb{Z}_{Z} \cup \mathbb{Z}_{W}$ e $\operatorname{logo} B(\mathbf{u}, \mathbf{u})=\sum_{j \in \mathbb{Z}_{Z} \cup \mathbb{Z}_{W}} \mu_{j} c_{j}^{2} \geq K_{a+b, \delta}\|\mathbf{u}\|_{E}^{2}$, provando (3.29).

Para provar (3.30) e (3.31) notemos que se $a+b>0$ e $\operatorname{dist}\left(a+b, \sigma(-\Delta) \backslash\left\{\lambda_{k}\right\}\right)>\alpha>0$, caso $k \geq 2$, temos

$$
\begin{aligned}
& \min _{i \in \mathbb{N} \backslash\{k, \ldots, k+m-1\}}\left|\mu_{i}\right|=\min \left\{\mu_{k+m},-\mu_{k-1}\right\}=\min \left\{1-\frac{a+b}{\lambda_{k+m}}, \frac{a+b}{\lambda_{k-1}}-1\right\}> \\
& \min \left\{\frac{\alpha}{\lambda_{k+m}}, \frac{\alpha}{\lambda_{k-1}}\right\}=\frac{\alpha}{\lambda_{k+m}} .
\end{aligned}
$$

No caso $k=1$, temos $\min _{i \in \mathbb{N} \backslash\{1\}}\left|\mu_{i}\right|=1-\frac{a+b}{\lambda_{2}} \geq \frac{\alpha}{\lambda_{2}}$.

Em ambos os casos, obtemos que $\left|\mu_{i}\right|$ é destacado de zero, uniformemente com respeito à soma $a+b$, para $i \in \mathbb{N} \backslash\{k, \ldots, k+m-1\}$. Assim existe $G_{\alpha, \delta}>0$ dependendo de $\alpha \mathrm{e}$ de $\delta$ (e de $k, l)$, tal que

$$
\left|\mu_{i}\right| \geq G_{\alpha, \delta} \text {, para todo } i \in \mathbb{Z}_{0} \backslash\{k, \ldots, k+m-1\},
$$

de onde segue que $B(\mathbf{u}, \mathbf{u}) \leq-G_{\alpha, \delta}\|\mathbf{u}\|_{E}^{2}$ para todo $\mathbf{u} \in V$ e $B(\mathbf{u}, \mathbf{u}) \geq G_{\alpha, \delta}\|\mathbf{u}\|_{E}^{2}$ para todo $\mathbf{u} \in W$.

Observação 3.12. Comparando as estimativas (3.35), (3.38) com (3.27), podemos ver que, uma vez fixados $\alpha$ e $\delta$, para $\varepsilon$ suficientemente pequeno, $\mu_{i}=\mu_{k}$ apenas para $i=$ $k, . ., k+m-1$, logo o subespaço $Z$ definido em (3.26) coincide com o definido no enunciado do Teorema 3.1 .

O Lema 3.10 é suficiente para obter as duas geometrias de ponto de sela para o funcional $J^{+}$, dadas pelas proposições a seguir. Na Figura 3.2 está esboçada uma representação destas geometrias.

Proposição 3.13. Sob as hipóteses do Teorema 3.1, se $\operatorname{dist}(a-b, \sigma(-\Delta))>\delta>0$, suponhamos $a+b \notin \sigma(-\Delta)$ e $\lambda_{k}$ é o primeiro autovalor maior que $a+b$, então existem 
$D_{a+b, \delta} \in \mathbb{R}$ e $\rho_{a+b, \delta}>0$, tais que

$$
\begin{array}{ll}
J^{+}(\mathbf{u}) \geq D_{a+b, \delta}, & \forall \mathbf{u} \in Z \oplus W, \\
J^{+}(\mathbf{u})<D_{a+b, \delta}, & \forall \mathbf{u} \in \rho S_{V}, \rho \geq \rho_{a+b, \delta} .
\end{array}
$$

Demonstração. Seja $\mathbf{u} \in Z \oplus W$. Por (3.29) e (3.9.

$$
J^{+}(\mathbf{u}) \geq K_{a+b, \delta}\|\mathbf{u}\|_{E}^{2}-S_{0}\left(1+\|\mathbf{u}\|_{E}^{q}\right)-2 H\|\mathbf{u}\|_{E} .
$$

Como $K_{a+b, \delta}>0$ e $q \in(1,2)$, a função de $\|\mathbf{u}\|_{E}$ acima é limitada inferiormente, logo existe $D_{a+b, \delta} \in \mathbb{R}$ satisfazendo (3.39).

Por outro lado, seja $\mathbf{u} \in V$. Por (3.28) e (3.9-3.10), temos

$$
J^{+}(\mathbf{u}) \leq-K_{a+b, \delta}\|\mathbf{u}\|_{E}^{2}+S_{0}\left(1+\|\mathbf{u}\|_{E}^{q}\right)+H\|\mathbf{u}\|_{E} \cdot
$$

Uma vez que $K_{a+b, \delta}>0$ e $q \in(1,2)$, a função acima tende a $-\infty$, quando $\|\mathbf{u}\|_{E} \rightarrow+\infty$, logo existe $\rho_{a+b, \delta}>0$ que satisfaz (3.40).

Observação 3.14. Observemos que, de fato, a hipótese (ff) (ou (F)) não foi usada na demonstração acima: ela é necessária apenas para a próxima proposição.

Proposição 3.15. Sob as hipóteses do Teorema [3.1, se dist $(a-b, \sigma(-\Delta))>\delta>0$ e $\lambda_{k}$ é o primeiro autovalor maior que $a+b$, então existe $\varepsilon_{0}>0$, dependendo de $\delta$, tal que se $a+b \in\left(\lambda_{k}-\varepsilon_{0}, \lambda_{k}\right)$, então existem $E_{\delta}, D_{a+b, \delta} \in \mathbb{R}, \rho_{a+b, \delta}>R_{\delta}>0$, tais que valem (3.39), 3.40) $e$

$$
\begin{aligned}
J^{+}(\mathbf{u}) \geq E_{\delta}, & \forall \mathbf{u} \in W, \\
J^{+}(\mathbf{u})<E_{\delta}-1, & \forall \mathbf{u} \in R_{\delta} S_{V Z}, \\
J^{+}(\mathbf{u})<E_{\delta}-1, & \forall \mathbf{u} \in V \operatorname{com}\|\mathbf{u}\|_{E}>R_{\delta} .
\end{aligned}
$$

Demonstração. Definamos $\alpha=\frac{1}{2} \operatorname{dist}\left(\lambda_{k}, \sigma(-\Delta) \backslash\left\{\lambda_{k}\right\}\right)$ e seja $a+b \in\left(\lambda_{k}-\varepsilon_{0}, \lambda_{k}\right)$, onde por enquanto pedimos apenas $\varepsilon_{0}<\alpha$, o que implica $\operatorname{dist}\left(a+b, \sigma(-\Delta) \backslash\left\{\lambda_{k}\right\}\right)>\alpha$.

- Seja $\mathbf{u} \in W$. Por (3.31) e (3.9)

$$
J^{+}(\mathbf{u}) \geq G_{\alpha, \delta}\|\mathbf{u}\|_{E}^{2}-S_{0}\left(1+\|\mathbf{u}\|_{E}^{q}\right)-H\|\mathbf{u}\|_{E}
$$


Figura 3.2: Duas geometrias de ponto de sela para o funcional $\mathrm{J}^{+}$

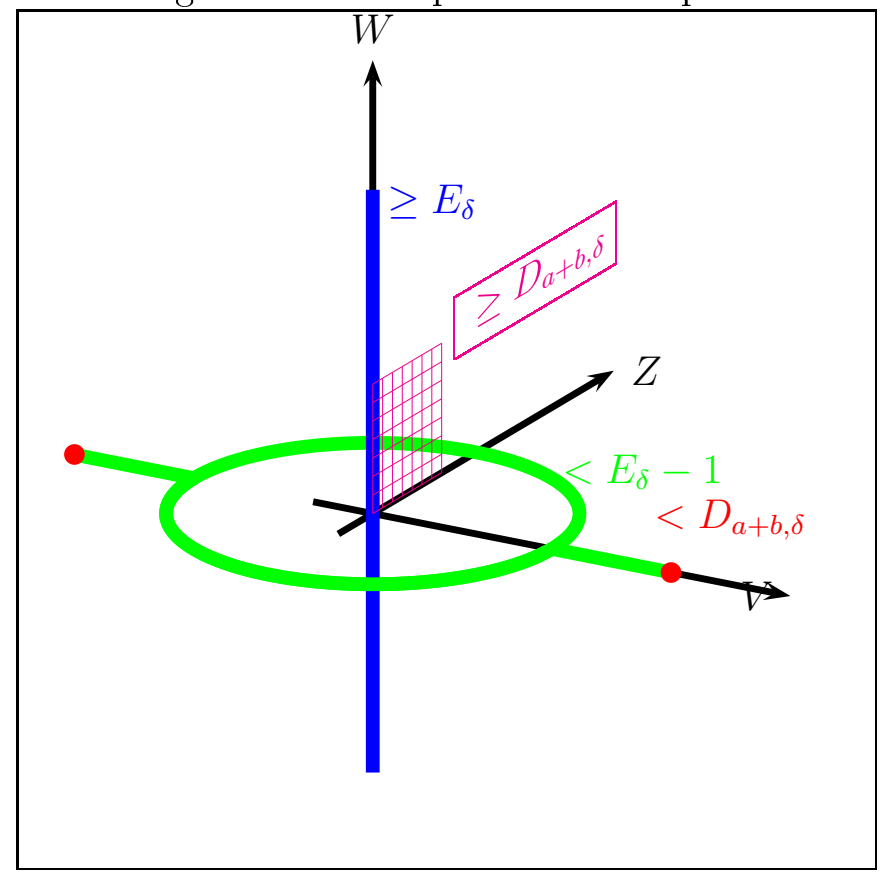

Uma vez que $G_{\alpha, \delta}>0$ e $q \in(1,2)$, existe $E_{\delta} \in \mathbb{R}$ satisfazendo (3.43). Notemos que a constante $E_{\delta}$ depende também de $\alpha$, que não aparece na notação uma vez que a este ponto podemos considerá-lo fixado.

- Seja agora $\mathbf{u} \in V$. Por $(\underline{3.30})$ e $(3.9+3.10)$, temos

$$
J^{+}(\mathbf{u}) \leq-G_{\alpha, \delta}\|\mathbf{u}\|_{E}^{2}+S_{0}\left(1+\|\mathbf{u}\|_{E}^{q}\right)+H\|\mathbf{u}\|_{E}
$$

Uma vez que $G_{\alpha, \delta}>0$ e $q \in(1,2)$, a estimativa (3.45) estará satisfeita desde que $R_{\delta}>\widetilde{R}$, onde $\widetilde{R}$ pode ser escolhido dependendo apenas de $\delta$ e $\alpha$, mas não de $a, b$.

- A fim de provar que existe $R_{\delta}>\widetilde{R}$, satisfazendo (3.44) para algum $\varepsilon_{0} \in(0, \alpha)$, suponhamos por contradição, que para quaisquer sequências $\varepsilon_{j} \rightarrow 0^{+}$e $\left\{R_{j}\right\}$, com $R_{j}>\widetilde{R}$ para todo $j \in \mathbb{N}$, existam $\mathbf{u}_{j} \in R_{j} S_{V Z}$ e $a_{j}, b_{j} \in \mathbb{R}$, tais que $a_{j}+b_{j} \in$ $\left(\lambda_{k}-\varepsilon_{j}, \lambda_{k}\right), \operatorname{dist}\left(a_{j}-b_{j}, \sigma(-\Delta)\right)>\delta \mathrm{e}$

$$
J_{a_{j}, b_{j}}^{+}\left(\mathbf{u}_{j}\right) \geq E_{\delta}-1
$$

Denotemos por $\left\{\mu_{i}^{j}\right\}_{i \in \mathbb{Z}_{0}}$ os autovalores de $B_{a_{j}, b_{j}}$, e observemos que as autofunções 
correspondentes não dependem de $j$.

Sem perda de generalidade, podemos supor que $R_{j} \rightarrow+\infty$ e que $\varepsilon_{j} R_{j}^{2} \rightarrow 0$. Escrevamos $\mathbf{u}_{j}=\mathbf{v}_{j}+\mathbf{z}_{j}$, onde $\mathbf{v}_{j} \in V$ e $\mathbf{z}_{j} \in Z$, para todo $j \in \mathbb{N}$.

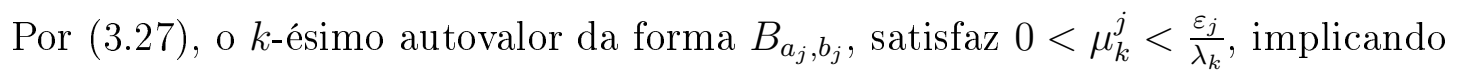

$$
B_{a_{j}, b_{j}}\left(\mathbf{z}_{j}, \mathbf{z}_{j}\right) \leq \frac{\varepsilon_{j}}{\lambda_{k}}\left\|\mathbf{z}_{j}\right\|_{E}^{2}
$$

Utilizando também (3.30), temos

$$
B_{a_{j}, b_{j}}\left(\mathbf{u}_{j}, \mathbf{u}_{j}\right)=B_{a_{j}, b_{j}}\left(\mathbf{z}_{j}, \mathbf{z}_{j}\right)+B_{a_{j}, b_{j}}\left(\mathbf{v}_{j}, \mathbf{v}_{j}\right) \leq \frac{\varepsilon_{j}}{\lambda_{k}}\left\|\mathbf{z}_{j}\right\|_{E}^{2}-G_{\alpha, \delta}\left\|\mathbf{v}_{j}\right\|_{E}^{2}
$$

e assim, para todo $j \in \mathbb{N}$, segue

$$
E_{\delta}-1 \leq J_{a_{j}, b_{j}}^{+}\left(\mathbf{u}_{j}\right) \leq \frac{\varepsilon_{j}}{\lambda_{k}}\left\|\mathbf{z}_{j}\right\|_{E}^{2}-G_{\alpha, \delta}\left\|\mathbf{v}_{j}\right\|_{E}^{2}-\mathcal{F}\left(\mathbf{u}_{j}\right)-\mathcal{H}\left(\mathbf{u}_{j}\right)
$$

Dividindo a equação (3.49) por $R_{j}^{2}$ e reordenando, temos

$$
\frac{G_{\alpha, \delta}}{R_{j}^{2}}\left\|\mathbf{v}_{j}\right\|_{E}^{2} \leq \frac{\varepsilon_{j}}{\lambda_{k} R_{j}^{2}}\left\|\mathbf{z}_{j}\right\|_{E}^{2}-\frac{E_{\delta}-1}{R_{j}^{2}}-\frac{\mathcal{F}\left(\mathbf{u}_{j}\right)+\mathcal{H}\left(\mathbf{u}_{j}\right)}{R_{j}^{2}} .
$$

Notemos que $\frac{\left|\mathcal{F}\left(\mathbf{u}_{j}\right)+\mathcal{H}\left(\mathbf{u}_{j}\right)\right|}{R_{j}^{2}} \leq \frac{2 C_{0}\left(1+\left\|\mathbf{u}_{j}\right\|_{\left[L^{2}\right]^{2}}^{q}\right)+2 H\left\|\mathbf{u}_{j}\right\|_{\left[L^{2}\right]^{2}}}{R_{j}^{2}} \leq \frac{C\left(1+R_{j}+R_{j}^{q}\right)}{R_{j}^{2}} \rightarrow 0$, quando $j \rightarrow \infty$, uma vez que $q \in(1,2)$. Além disso $\frac{E_{\delta}-1}{R_{j}^{2}} \rightarrow 0$ e $\frac{\varepsilon_{j}}{\lambda_{k} R_{j}^{2}}\left\|\mathbf{z}_{j}\right\|_{E}^{2} \leq \frac{\varepsilon_{j}}{\lambda_{k}} \rightarrow 0$, quando $j \rightarrow \infty$. Portanto segue que

$$
\frac{\left\|\mathbf{v}_{j}\right\|_{E}}{R_{j}} \rightarrow 0
$$

e como $\left\|\mathbf{v}_{j}\right\|_{E}^{2}=R_{j}^{2}-\left\|\mathbf{z}_{j}\right\|_{E}^{2}$, temos também

$$
\frac{\left\|\mathbf{z}_{j}\right\|_{E}}{R_{j}} \rightarrow 1
$$

Agora, para cada $j \in \mathbb{N}$, definamos $\widehat{\mathbf{z}}_{j}=\frac{\mathbf{z}_{j}}{R_{j}}, \widehat{\mathbf{v}}_{j}=\frac{\mathbf{v}_{j}}{R_{j}}$ e $\widehat{\mathbf{u}}_{j}=\widehat{\mathbf{z}}_{j}+\widehat{\mathbf{v}}_{j}$. Assim, por (3.51) e (3.52), existe $\widehat{\mathbf{z}}_{0} \in Z$ com $\left\|\widehat{\mathbf{z}}_{0}\right\|_{E}=1$, tal que, passando a subsequência, $\widehat{\mathbf{v}}_{j} \rightarrow 0$ em $E, \widehat{\mathbf{z}}_{j} \rightarrow \widehat{\mathbf{z}}_{0}$ em $E$ e uniformemente (pois a dimensão de $Z$ é finita), 
quando $j \rightarrow \infty$. Denotemos por $P_{1} \mathbf{u}$ e $P_{2} \mathbf{u}$ as componentes de um vetor $\mathbf{u} \in E$, isto é, $\mathbf{u}=\left(P_{1} \mathbf{u}, P_{2} \mathbf{u}\right)$. Como $\left\|\widehat{\mathbf{z}}_{0}\right\|_{E}=1$, segue que pelo menos uma das componentes $P_{1} \widehat{\mathbf{u}}_{j}$ ou $P_{2} \widehat{\mathbf{u}}_{j}$ não converge a zero em $E$. Sem perda de generalidade, suponhamos que $P_{1} \widehat{\mathbf{u}}_{j}$ é tal que não converge a zero em $E$. Assim temos

$$
\left\{\begin{array}{l}
P_{1} \widehat{\mathbf{z}}_{j} \stackrel{j \rightarrow \infty}{\longrightarrow} P_{1} \widehat{\mathbf{z}}_{0} \neq 0, \text { uniformemente em } \Omega \\
P_{1} \widehat{\mathbf{v}}_{j} \stackrel{j \rightarrow \infty}{\longrightarrow} 0, \text { em } L^{2}(\Omega)
\end{array}\right.
$$

Desta forma, existem $\chi>0$ e $j_{0} \in \mathbb{N}$, tais que para todo $j>j_{0}$, os conjuntos $\Omega_{j}:=\left\{x \in \Omega:\left|P_{1} \widehat{\mathbf{u}}_{j}(x)\right|>\chi\right\}$ satisfazem $\left|\Omega_{j}\right|>\chi$. De fato, por (3.53), existe $\chi>0$ tal que, para $j$ suficientemente grande, existem conjuntos $\widetilde{\Omega}_{j} \operatorname{com}\left|\widetilde{\Omega}_{j}\right|>\chi$, tais que $\left|P_{1} \widehat{\mathbf{z}}_{j}(x)\right|>2 \chi$ e $\left|P_{1} \widehat{\mathbf{v}}_{j}(x)\right|<\chi$, q. t. p. em $\widetilde{\Omega}_{j}$, e portanto $\left|P_{1} \widehat{\mathbf{u}}_{j}(x)\right|>\chi$ q. t. p. em $\widetilde{\Omega}_{j}$.

Agora precisamos considerar separadamente as hipóteses $(\mathbf{f})$ e $(\mathbf{F})$.

Se as hipóteses $(\underline{1.2})$ e $(\underline{\mathbf{f}})$ ocorrem, dado $M>0$, existe $C_{M} \in \mathbb{R}$, tal que $F_{1,2}(x, t) \geq$ $M|t|-C_{M}$. Então, tomando $M=\frac{1+H}{\chi^{2}}$, temos para $j>j_{0}$, a estimativa

$$
\int_{\Omega} F_{2}\left(x, R_{j}\left(P_{1} \widehat{\mathbf{u}}_{j}\right)\right) \geq M R_{j} \int_{\Omega}\left|P_{1} \widehat{\mathbf{u}}_{j}\right|-D_{M} \geq M R_{j} \chi^{2}-D_{M},
$$

onde $D_{M}=\int_{\Omega} C_{M}$. Além disso, uma vez que $F_{1}\left(x, P_{2} \widehat{\mathbf{u}}_{j}\right) \geq-C_{M}$,

$$
\int_{\Omega} F_{1}\left(x, R_{j}\left(P_{2} \widehat{\mathbf{u}}_{j}\right)\right) \geq-D_{M}
$$

Portanto, segue destas duas estimativas e de (3.9)

$$
\mathcal{F}\left(R_{j} \widehat{\mathbf{u}}_{j}\right)+\mathcal{H}\left(R_{j} \widehat{\mathbf{u}}_{j}\right) \geq M R_{j} \chi^{2}-2 D_{M}-R_{j} H=R_{j}-2 D_{M} .
$$

Deste modo, de (3.49), uma vez que $R_{j} \widehat{\mathbf{u}}_{j}=\mathbf{u}_{j}$, segue

$$
E_{\delta}-1 \leq \frac{\varepsilon_{j}}{\lambda_{k}}\left\|R_{j} \widehat{\mathbf{z}}_{j}\right\|_{E}^{2}-G_{\alpha, \delta}\left\|R_{j} \widehat{\mathbf{v}}_{j}\right\|_{E}^{2}-\mathcal{F}\left(R_{j} \widehat{\mathbf{u}}_{j}\right)-\mathcal{H}\left(R_{j} \widehat{\mathbf{u}}_{j}\right),
$$


e por (3.56), temos

$$
E_{\delta}-1 \leq \frac{\varepsilon_{j}}{\lambda_{k}}\left\|R_{j} \widehat{\mathbf{z}}_{j}\right\|_{E}^{2}-G_{\alpha, \delta}\left\|R_{j} \widehat{\mathbf{v}}_{j}\right\|_{E}^{2}-R_{j}+2 D_{M}
$$

e $\log 0$

$$
G_{\alpha, \delta} R_{j}^{2}\left\|\widehat{\mathbf{v}}_{j}\right\|_{E}^{2}+R_{j} \leq \frac{\varepsilon_{j} R_{j}^{2}}{\lambda_{k}}+2 D_{M}-E_{\delta}+1
$$

o que é uma contradição, pois como $\varepsilon_{j} R_{j}^{2} \rightarrow 0$, quando $j \rightarrow \infty$, o lado direito da desigualdade permanece limitado, enquanto o lado esquerdo $G_{\alpha, \delta} R_{j}^{2}\left\|\widehat{\mathbf{v}}_{j}\right\|_{E}^{2}+R_{j} \rightarrow$ $+\infty$, quando $j \rightarrow \infty$, uma vez que $R_{j} \rightarrow \infty$.

Concluímos que existem $R_{\delta}>\widetilde{R}$ e $\varepsilon_{0} \in(0, \alpha)$ satisfazendo (3.44).

Agora consideramos o caso em que sejam válidas as hipóteses $(1.2)$ e $(\underline{\mathbf{F}})$. Começamos mostrando que

$$
\lim _{R \rightarrow \infty} \inf _{j>j_{0}} \int_{\Omega} F_{2}\left(x, R P_{1}\left(\widehat{\mathbf{u}}_{j}\right)\right)=+\infty .
$$

De fato, mostremos que dado $M>0$, existe $R_{0}$ suficientemente grande, tal que

$$
\int_{\Omega} F_{2}\left(x, R P_{1}\left(\widehat{\mathbf{u}}_{j}\right)\right) \geq M, \quad \forall j \geq j_{0} \text { e } R \geq R_{0}
$$

Primeiramente notemos que existe $C_{F}>0$, tal que

$$
F_{i}(x, t) \geq-C_{F} \text {, para todo }(x, t) \in \Omega \times \mathbb{R} \text { e } i=1,2 ;
$$

de fato, por $(\underline{\mathbf{F}}-i)$ existe $t_{0}>0$ tal que $F_{i}(x, t) \geq 0$ para $|t|>t_{0}$ e por (1.2) $F_{i}(x, t) \geq-S\left(t_{0}+t_{0}^{q} / q\right)$ para $|t| \leq t_{0}$.

Então, definamos $M_{1}=\frac{M+|\Omega| C_{F}}{\chi}$. Por $(\underline{\mathbf{F}-i)})$, existe $s_{0}>0$ tal que,

$$
F_{2}(x, s)>M_{1}, \quad \forall|s|>s_{0} .
$$

Notemos também que para todo $R>\frac{s_{0}}{\chi}$, vale $\Omega_{j} \subseteq\left\{x \in \Omega:\left|R P_{1}\left(\widehat{\mathbf{u}}_{j}(x)\right)\right|>s_{0}\right\}$. 
Então, segue que

$$
\begin{aligned}
\int_{\Omega} F_{2}\left(x, R P_{1}\left(\widehat{\mathbf{u}}_{j}\right)\right) & \geq \int_{\Omega_{j}} F_{2}\left(x, R P_{1}\left(\widehat{\mathbf{u}}_{j}\right)\right)+\int_{\Omega \backslash \Omega_{j}}-C_{F} \\
& \geq \int_{\Omega_{j}} M_{1}-C_{F}|\Omega| \geq M_{1} \chi-C_{F}|\Omega|=M .
\end{aligned}
$$

Portanto, (3.60) está satisfeita, e uma vez que $\int_{\Omega} F_{1}\left(x, R P_{2}\left(\widehat{\mathbf{u}}_{j}\right)\right)$ é limitado inferiormente, segue que

$$
\mathcal{F}\left(\mathbf{u}_{j}\right)=\mathcal{F}\left(R_{j} \widehat{\mathbf{u}}_{j}\right) \rightarrow+\infty, \text { quando } \quad j \rightarrow+\infty
$$

Além disso, pela Observação 3.12 e $(\mathbf{F}-i i)$, temos $\mathcal{H}\left(\mathbf{u}_{j}\right)=\mathcal{H}\left(\mathbf{v}_{j}\right)$, e então

$$
G_{\alpha, \delta}\left\|\mathbf{v}_{j}\right\|_{E}^{2}+\mathcal{H}\left(\mathbf{u}_{j}\right)=G_{\alpha, \delta}\left\|\mathbf{v}_{j}\right\|_{E}^{2}+\mathcal{H}\left(\mathbf{v}_{j}\right) \geq G_{\alpha, \delta}\left\|\mathbf{v}_{j}\right\|_{E}^{2}-H\left\|\mathbf{v}_{j}\right\|_{E}
$$

Logo, existe $\delta_{2}>0$, tal que $G_{\alpha, \delta}\left\|\mathbf{v}_{j}\right\|_{E}^{2}+\mathcal{H}\left(\mathbf{u}_{j}\right) \geq-\delta_{2}$.

Portanto, em (3.49), temos

$$
\mathcal{F}\left(\mathbf{u}_{j}\right) \leq \frac{\varepsilon_{j}\left\|\mathbf{z}_{j}\right\|_{E}^{2}}{\lambda_{k}}-E_{\delta}+1+\delta_{2}
$$

o que é uma contradição, como no caso anterior, uma vez que o lado esquerdo tende a $+\infty$, enquanto o lado direito permanece limitado.

Concluímos assim que existem $R_{\delta}>\widetilde{R}$ e $\varepsilon_{0} \in(0, \alpha)$ que satisfazem (3.44).

As constantes do enunciado da proposição podem ser obtidas da seguinte forma: primeiramente tomamos $E_{\delta} \in \mathbb{R}$ satisfazendo (3.43), e então obtemos $\varepsilon_{0}>0$ e $R_{\delta}>0$, satisfazendo (3.44) e (3.45). Estas estimativas valem uniformemente para $a+b \in\left(\lambda_{k}-\varepsilon_{0}, \lambda_{k}\right)$. Assim, fixando $a+b$ neste intervalo, podemos obter a constante $D_{a+b, \delta} \in \mathbb{R}$ que satisfaz (3.39). Finalmente, obtemos $\rho_{a+b, \delta}>0$ que satisfaz (3.40); observemos que podemos tomar $\rho_{a+b, \delta}>R_{\delta}$, como desejado.

Observação 3.16. A Proposição 3.13 vale para qualquer valor de $a+b$ fixado no intervalo $\left(\lambda_{k-1}, \lambda_{k}\right)$. Esta geometria produzirá (veja Seções 3.2.2 e 3.2.4) uma solução para o problema $(1.1+)$. Por outro lado, a geometria da Proposição 3.15 vale para $a+b$ perto 
do autovalor $\lambda_{k}$ por baixo. Esta outra geometria também dará uma solução para o problema $(1.1+)$. Logo para tais valores de $a+b$, ambas geometrias valerão, e então teremos duas soluções do problema $(\underline{1.1+})$. No entanto, ainda precisaremos mostrar que elas são distintas.

\subsubsection{Obtendo duas sequências de pontos críticos}

Como observado na introdução do capítulo, o funcional $J^{+}$definido em (3.5) , é fortemente indefinido e desta forma recorremos a uma aproximação finito dimensional para poder utilizar o Teorema de Ponto de Sela.

Definamos então, para cada $n>k+m$,

$$
\begin{gathered}
E_{n}=\operatorname{span}\left[\boldsymbol{\psi}_{-n}, \ldots, \boldsymbol{\psi}_{n}\right] \subseteq E, \\
V_{n}=V \cap E_{n} \quad \text { e } \quad W_{n}=W \cap E_{n} .
\end{gathered}
$$

Pela Observação [3.12, podemos assumir que $Z \subseteq E_{n}$, para todo $n>k+m$. Denotaremos por $B_{V}^{n}, B_{V Z}^{n}$ e $B_{Z W}^{n}$ as bolas unitárias fechadas, com respeito à norma de $E$, nos subespaços $V_{n}, V_{n} \oplus Z$ e $Z \oplus W_{n}$, e por $S_{V}^{n}, S_{V Z}^{n}$ e $S_{Z W}^{n}$ suas respectivas fronteiras relativas. Por fim, seja $J_{n}^{+}$o funcional $J^{+}$restrito ao subespaço $E_{n}$.

Sob as hipóteses do Teorema [3.1, valem as afirmações da Proposição 3.15. Então, fixados $a, b$ tais que $\operatorname{dist}(a-b, \sigma(-\Delta))>\delta>0$ e $a+b \in\left(\lambda_{k}-\varepsilon_{0}, \lambda_{k}\right)$, o funcional $J_{n}^{+}$ satisfaz as mesmas estimativas nos subespaços $V_{n}, Z$ e $W_{n}$.

Além disso, o seguinte lema (cuja demonstração deixamos para a Seção 3.4) implica que os funcionais $J_{n}^{+}$satisfazem a condição (PS).

Lema 3.17. Sejam $a \pm b \notin \sigma(-\Delta), f_{1}, f_{2}$ satisfazendo (1.2) e $h_{1}, h_{2} \in L^{2}(\Omega)$. Fixemos $n>k+m$ e seja $\left\{\mathbf{u}_{j}\right\} \subset E_{n}$ uma sequência, tal que

$$
\left|\left(J_{n}^{ \pm}\right)^{\prime}\left(\mathbf{u}_{j}\right)[\phi]\right| \leq \varepsilon_{i}\|\phi\|_{E}, \quad \text { para todo } \phi \in E_{n}
$$

onde $\varepsilon_{j} \rightarrow 0$ quando $j \rightarrow \infty$. Então $\left\{\mathbf{u}_{j}\right\}$ possui uma subsequência convergente em $E_{n}$.

Nestas condições, para todo $n>k+m$, podemos aplicar duas vezes o Teorema do Ponto de Sela: de fato, as estimativas (3.39) e (3.40) definem uma geometria de sela entre os subespaços $V_{n}$ e $Z \oplus W_{n}$, enquanto as desigualdades (3.43) e (3.44) definem uma outra 
geometria de sela, entre os subespaços $V_{n} \oplus Z$ e $W_{n}$. Obtemos então a existência dos pontos críticos $\mathbf{u}_{n}^{+}, \mathbf{v}_{n}^{+} \in E_{n}$ dos funcionais $J_{n}^{+}$, nos níveis críticos

$$
\begin{aligned}
& c_{n}^{+}=\inf _{\gamma \in \Gamma_{V}^{n}} \sup _{\mathbf{u} \in \rho_{a+b, \delta} B_{V}^{n}} J_{n}^{+}(\gamma(\mathbf{u})) \geq D_{a+b, \delta}, \\
& d_{n}^{+}=\inf _{\gamma \in \Gamma_{V Z}^{n}} \sup _{\mathbf{v} \in R_{\delta} B_{V Z}^{n}} J_{n}^{+}(\gamma(\mathbf{v})) \geq E_{\delta},
\end{aligned}
$$

onde

$$
\begin{aligned}
\Gamma_{V}^{n} & =\left\{\gamma \in \mathcal{C}\left(\rho_{a+b, \delta} B_{V}^{n}, E_{n}\right): \gamma(\mathbf{u})=\mathbf{u} \text { se }\|\mathbf{u}\|_{E}=\rho_{a+b, \delta}\right\}, \\
\Gamma_{V Z}^{n} & =\left\{\gamma \in \mathcal{C}\left(R_{\delta} B_{V Z}^{n}, E_{n}\right): \gamma(\mathbf{v})=\mathbf{v} \text { se }\|\mathbf{v}\|_{E}=R_{\delta}\right\} .
\end{aligned}
$$

Como $\mathbf{u}_{n}^{+}, \mathbf{v}_{n}^{+} \in E_{n}$ são pontos críticos do funcional $J^{+}$restrito a subespaços, não correspondem a soluções do nosso problema. As soluções que encontraremos, na Seção 3.2.4, serão pontos críticos do funcional $J^{+}$cujos níveis serão limites de subsequências dos níveis $c_{n}^{+}$e $d_{n}^{+}$encontrados. Precisamos então de estimativas sobre estes níveis que garantam a existência de subsequências convergentes, e também que os níveis limites sejam distintos. Estas estimativas são dadas no seguinte lema.

Lema 3.18. Sob as condições da Proposição 3.15, fixados a, b tais que

$$
\operatorname{dist}(a-b, \sigma(-\Delta))>\delta>0 \quad e \quad a+b \in\left(\lambda_{k}-\varepsilon_{0}, \lambda_{k}\right),
$$

existe $T_{\delta}>0$, tal que $c_{n}^{+} \in\left[D_{a+b, \delta}, E_{\delta}-1\right]$ e $d_{n}^{+} \in\left[E_{\delta}, T_{\delta}\right]$, para todo $n>k+m$.

Demonstração. As limitações inferiores estão contidas em (3.68] 3.69), e seguem diretamente do Teorema do Ponto de Sela.

Para as limitações superiores, notemos que é possível construir $\gamma_{0} \in \Gamma_{V}^{n}$ de modo que $\gamma_{0}\left(\rho_{a+b, \delta} B_{V}^{n}\right)$ seja a reunião da coroa circular $\left\{\mathbf{u} \in V_{n}:\|\mathbf{u}\|_{E} \in\left[R_{\delta}, \rho_{a+b, \delta}\right]\right\}$ com uma semiesfera contida em $R_{\delta} S_{V Z}^{n}$, com bordo $R_{\delta} S_{V}^{n}$ : por exemplo, podemos tomar

$$
\gamma_{0}(p)= \begin{cases}p ; & \text { se } R_{\delta} \leq\|p\|_{E} \leq \rho_{a+b, \delta} \\ p+\sqrt{\left(R_{\delta}\right)^{2}-\|p\|_{E}^{2}} \psi_{k} ; & \text { se }\|p\|_{E} \leq R_{\delta}\end{cases}
$$

Desta forma, por (3.44) e (3.45), temos $\sup _{\mathbf{u} \in \rho_{a+b, \delta} B_{V}^{n}} J_{n}^{+}\left(\gamma_{0}(\mathbf{u})\right)<E_{\delta}-1$, mostrando que $c_{n}^{+}<E_{\delta}-1$. 
Por outro lado, uma vez que a aplicação identidade pertence a $\Gamma_{V Z}^{n}$, temos $d_{n}^{+} \leq$ $\sup _{\mathbf{v} \in R_{\delta} B^{n} z} J^{+}(\mathbf{v})$. Se $\mathbf{u} \in V \oplus Z$, sejam $\mathbf{z} \in Z$ e $\mathbf{v} \in V$ tais que $\mathbf{u}=\mathbf{z}+\mathbf{v}$. Como $v \in R_{\delta} B_{V Z}^{n}$

$a+b \in\left(\lambda_{k}-\varepsilon_{0}, \lambda_{k}\right)$, vale

$$
-K_{a+b, \delta}<0<\mu_{k} \leq \frac{\varepsilon_{0}}{\lambda_{k}}
$$

Então, por (3.72), (3.28) e (3.9- $\sqrt{3.10})$, temos

$$
\begin{aligned}
J^{+}(\mathbf{u}) & \leq \frac{\varepsilon_{0}}{\lambda_{k}}\|\mathbf{z}\|_{E}^{2}-K_{a+b, \delta}\|\mathbf{v}\|_{E}^{2}+S_{0}\left(1+\|\mathbf{u}\|_{E}^{q}\right)+H\|\mathbf{u}\|_{E} \\
& \leq \frac{\varepsilon_{0}}{\lambda_{k}}\|\mathbf{u}\|_{E}^{2}+S_{0}\left(1+\|\mathbf{u}\|_{E}^{q}\right)+H\|\mathbf{u}\|_{E},
\end{aligned}
$$

implicando que $J^{+}$é limitado superiormente (uniformemente com respeito a $n$ ), nos conjuntos limitados $R_{\delta} B_{V Z}^{n}$. Concluímos que existe $T_{\delta}>0$ que limita $d_{n}^{+}$superiormente.

\subsubsection{As geometrias de ponto de sela quando $a+b$ é quase resso- nante superiormente}

Nesta seção mostramos como obter níveis críticos análogos a (3.68) e (3.69), no caso (b) do Teorema 3.1, adaptando os argumentos utilizados na Seção 3.2.1 para o caso (a).

Consideremos o funcional $J^{-}(\mathbf{u})$ definido em (3.5), que é igual ao funcional $J^{+}$exceto por ter a forma $(-B)$ no lugar de $B$. Assim, definamos as duas sequências de autovalores de $(-B)$

$$
\widetilde{\mu}_{ \pm i}=-\mu_{ \pm i}=-\frac{-b \pm\left(\lambda_{i}-a\right)}{\lambda_{i}}, i \in \mathbb{N}
$$

associados às mesmas autofunções $\boldsymbol{\psi}_{ \pm i}=\frac{\left(\phi_{i}, \pm \phi_{i}\right)}{\sqrt{2 \lambda_{i}}}, i \in \mathbb{N}$.

Então definamos os subespaços $\widetilde{V}, \widetilde{Z}$ e $\widetilde{W}$ da mesma forma que em (3.26), mas usando os autovalores $\widetilde{\mu}_{i}$ ao invés de $\mu_{i}, i \in \mathbb{Z}_{0}$.

Observemos que se $a+b \in\left(\lambda_{k}, \lambda_{k}+\varepsilon\right)$, para algum $\varepsilon>0$, então

$$
0<\widetilde{\mu}_{k}=\frac{(a+b)-\lambda_{k}}{\lambda_{k}}<\frac{\varepsilon}{\lambda_{k}}
$$

análoga a (3.27).

Precisamos apenas mostrar que o Lema 3.10 vale para $(-B)$ com a nova definição dos subespaços, pois o resto do argumento segue de maneira análoga à feita na Seção 3.2.1. 
Lema 3.19. Suponhamos $a \pm b \notin \sigma(-\Delta)$ e fixemos $\lambda_{k}$ o último autovalor menor que $a+b$, de multiplicidade $m$, e $\lambda_{l}$ o primeiro autovalor maior de $a-b$.

Se $\operatorname{dist}(a-b, \sigma(-\Delta))>\delta>0$, então existe uma constante $K_{a+b, \delta}>0$, dependendo da soma $a+b$ e de $\delta$, tal que

$$
\begin{array}{ll}
-B(\mathbf{u}, \mathbf{u}) \leq-K_{a+b, \delta}\|\mathbf{u}\|_{E}^{2}, & \forall \mathbf{u} \in \widetilde{V}, \\
-B(\mathbf{u}, \mathbf{u}) \geq K_{a+b, \delta}\|\mathbf{u}\|_{E}^{2}, & \forall \mathbf{u} \in \widetilde{Z} \oplus \widetilde{W}
\end{array}
$$

Além disso, se $a+b$ está suficientemente perto de $\lambda_{k}$ (em particular, se dist $(a+$ $\left.\left.b, \sigma(-\Delta) \backslash\left\{\lambda_{k}\right\}\right)>\alpha>0\right)$, então existe $G_{\alpha, \delta}>0$, dependendo de $\alpha$ e $\delta$, tal que

$$
\begin{array}{ll}
-B(\mathbf{u}, \mathbf{u}) \leq-G_{\alpha, \delta}\|\mathbf{u}\|_{E}^{2}, & \forall \mathbf{u} \in \widetilde{V} \\
-B(\mathbf{u}, \mathbf{u}) \geq G_{\alpha, \delta}\|\mathbf{u}\|_{E}^{2}, & \forall \mathbf{u} \in \widetilde{W}
\end{array}
$$

Demonstração. A demonstração deste lema segue como a do Lema 3.10.

De fato, considerando os autovalores da forma $(-B)$, a sequência $\widetilde{\mu}_{i}$, com $i \in \mathbb{N}$, é decrescente enquanto a sequência $\widetilde{\mu}_{-i}$, com $i \in \mathbb{N}$, é crescente. Assim, podemos mostrar como antes que vale uma estimativa como (3.36) para estes novos autovalores. Então (3.74) e (3.75) são válidas, pela definição de $\widetilde{V}, \widetilde{Z}$ e $\widetilde{W}$.

Considerando $a+b$ suficientemente perto de $\lambda_{k}$, obtemos, como no Lema 3.10, a existência de uma constante positiva $G_{\alpha, \delta}$, satisfazendo (3.76) e (3.77).

Pelo mesmo raciocínio usado na Observação 3.12, se $\varepsilon$ é suficientemente pequeno, o subespaço $\widetilde{Z}$ coincide com o subespaço $Z$ definido no enunciado do Teorema 3.1, Então, o Lema 3.19 e a estimativa (3.73) nos permitem mostrar resultados análogos às Proposições 3.13 e 3.15 para o funcional $J^{-}$, com os novos subespaços $\widetilde{V}, \widetilde{Z}$ e $\widetilde{W}$.

Proposição 3.20. Sob as hipóteses do Teorema 3.1, se dist $(a-b, \sigma(-\Delta))>\delta>0$, suponhamos $a+b \notin \sigma(-\Delta)$ e $\lambda_{k}$ é o último autovalor menor que $a+b$, então existem $D_{a+b, \delta} \in \mathbb{R}$ e $\rho_{a+b, \delta}>0$, tais que $J^{-}$satisfaz (3.39) e (3.40) nos subespaços $\widetilde{V}, \widetilde{Z}$ e $\widetilde{W}$.

Proposição 3.21. Sob as hipóteses do Teorema 3.1, se dist $(a-b, \sigma(-\Delta))>\delta>0$ e $\lambda_{k}$ é o primeiro autovalor menor que $a+b$, então existe $\varepsilon_{1}>0$, dependendo de $\delta$, tal que se $a+b \in\left(\lambda_{k}, \lambda_{k}+\varepsilon_{1}\right)$, então existem $E_{\delta}, D_{a+b, \delta} \in \mathbb{R}, \rho_{a+b, \delta}>R_{\delta}>0$, tais que $J^{-}$satisfaz (3.39), 3.40), (3.43), (3.44) e (3.45) nos subespaços $\widetilde{V}, \widetilde{Z}$ e $\widetilde{W}$. 
As Proposições 3.20 e 3.21 são suficientes para garantir, como na Seção 3.2.2, a existência de duas sequências de pontos críticos $\left\{\mathbf{u}_{n}^{-}\right\}$e $\left\{\mathbf{v}_{n}^{-}\right\}$, dos funcionais $J_{n}^{-}=\left.J^{-}\right|_{E_{n}}$, em níveis críticos $c_{n}^{-}, d_{n}^{-}$, análogos a (3.68) e (3.69).

\subsubsection{Existência de soluções no limite}

Nesta seção concluímos a demonstração do Teorema 3.1, mostrando que, passando a subsequência, as sequências de pontos críticos $\left\{\mathbf{u}_{n}^{+}\right\}$e $\left\{\mathbf{v}_{n}^{+}\right\}$, obtidas na Seção 3.2.1, convergem para soluções distintas de $(1.1+)$, enquanto $\left\{\mathbf{u}_{n}^{-}\right\}$e $\left\{\mathbf{v}_{n}^{-}\right\}$, da Seção 3.2.3, convergem para soluções distintas de (1.1-). Por fim, apresentamos a demonstração do Teorema 3.2 .

Antes de apresentar a demonstração do teorema, enunciamos um lema que está demonstrado na Seção 3.4 .

Lema 3.22. Sejam $a \pm b \notin \sigma(-\Delta), f_{1}, f_{2}$ satisfazendo (1.2) e $h_{1}, h_{2} \in L^{2}(\Omega)$. Se uma sequência $\left\{\mathbf{u}_{j}\right\} \subset E$, com $\mathbf{u}_{j} \in E_{j}$ para todo $j \in \mathbb{N}$, satisfaz

$$
\left|\left(J_{j}^{ \pm}\right)^{\prime}\left(\mathbf{u}_{j}\right)[\phi]\right| \leq \varepsilon_{j}\|\phi\|_{E}, \quad \text { para todo } \phi \in E_{j},
$$

onde $\varepsilon_{j} \rightarrow 0$, então $\left\{\mathbf{u}_{j}\right\}$ é uma sequência limitada em $E$.

Agora estamos em condições de demonstrar o Teorema 3.1 .

Demonstração do Teorema 3.1. Demonstramos apenas o item (a), uma vez que o item

(b) decorre pelo mesmo argumento, aplicado às sequências obtidas na Seção 3.2.3.

Primeiramente, notemos que pelo Lema 3.18, existem $c^{+} \in\left[D_{a+b, \delta}, E_{\delta}-1\right]$ e $d^{+} \in$ $\left[E_{\delta}, T_{\delta}\right]$ tais que, passando a uma subsequência, $c_{n}^{+} \rightarrow c^{+}$e $d_{n}^{+} \rightarrow d^{+}$, quando $n \rightarrow \infty$. Mostremos que existe $\mathbf{u}^{+} \in E$, ponto crítico do funcional $J^{+}$, tal que $J^{+}\left(\mathbf{u}^{+}\right)=c^{+}$. Pelo Teorema do Ponto de Sela, sabemos que para cada $n>k+m$, temos

$$
\begin{gathered}
J_{n}^{+}\left(\mathbf{u}_{n}^{+}\right)=c_{n}^{+}, \\
\left(J_{n}^{+}\right)^{\prime}\left(\mathbf{u}_{n}^{+}\right)[\boldsymbol{\phi}]=0, \quad \text { para todo } \boldsymbol{\phi} \in E_{n} .
\end{gathered}
$$

Como consequência, podemos aplicar o Lema 3.22, do qual segue que a sequência $\left\{\mathbf{u}_{n}^{+}\right\}$é 
limitada na norma $E$ e logo existe $\mathbf{u}^{+} \in E$ tal que, passando novamente a subsequência,

$$
\left\{\begin{array}{l}
\mathbf{u}_{n}^{+}=\left(u_{n}, v_{n}\right) \rightarrow \mathbf{u}^{+}=(u, v), \text { em } E \\
\mathbf{u}_{n}^{+}=\left(u_{n}, v_{n}\right) \rightarrow \mathbf{u}^{+}=(u, v), \text { em } L^{2} \times L^{2}
\end{array}\right.
$$

Seja $h$ um número natural qualquer maior que $k+m$. Testando (3.80) com $(0, \psi) \in E_{h}$ e $(\phi, 0) \in E_{h}$, temos respectivamente, para $n>h$,

$$
\left\{\begin{array}{l}
\int_{\Omega} \nabla u_{n} \nabla \psi-a \int_{\Omega} u_{n} \psi-b \int_{\Omega} v_{n} \psi-\int_{\Omega} f_{1}\left(x, v_{n}\right) \psi-\int_{\Omega} h_{1} \psi=0 \\
\int_{\Omega} \nabla v_{n} \nabla \phi-a \int_{\Omega} v_{n} \phi-b \int_{\Omega} u_{n} \phi-\int_{\Omega} f_{2}\left(x, u_{n}\right) \phi-\int_{\Omega} h_{2} \phi=0
\end{array}\right.
$$

Por (3.81) e pela continuidade dos operadores de Nemytskii associados a $f_{1}$ e $f_{2}$ (veja o Lema (3.9), temos no limite

$$
\left\{\begin{array}{l}
\int_{\Omega} \nabla u \nabla \psi-a \int_{\Omega} u \psi-b \int_{\Omega} v \psi-\int_{\Omega} f_{1}(x, v) \psi-\int_{\Omega} h_{1} \psi=0 \\
\int_{\Omega} \nabla v \nabla \phi-a \int_{\Omega} v \phi-b \int_{\Omega} u \phi-\int_{\Omega} f_{2}(x, u) \phi-\int_{\Omega} h_{2} \phi=0
\end{array}\right.
$$

isto é, $\left(J^{+}\right)^{\prime}\left(\mathbf{u}^{+}\right)[\phi]=0$, para todo $\phi=(\phi, \psi) \in E_{h}$. Como $\bigcup_{h \in \mathbb{N}} E_{h}$ é denso em $E$, segue que $\left(J^{+}\right)^{\prime}\left(\mathbf{u}^{+}\right)=0$, portanto $\mathbf{u}^{+}=(u, v)$ é ponto crítico do funcional $J^{+}$.

Mostremos agora que $\mathbf{u}_{n}^{+} \rightarrow \mathbf{u}^{+}$, fortemente em $E$, o que implica $J^{+}\left(\mathbf{u}_{n}^{+}\right) \rightarrow J^{+}\left(\mathbf{u}^{+}\right)$. Seja $P_{n}: H_{0}^{1}(\Omega) \rightarrow \operatorname{span}\left\{\phi_{1}, \ldots, \phi_{n}\right\}$ a projeção ortogonal. Assim $P_{n} u \rightarrow u, P_{n} v \rightarrow v$ em $H_{0}^{1}(\Omega)$ e como $\left(v_{n}-P_{n} v, u_{n}-P_{n} u\right) \in E_{n}$, podemos usá-la como função teste em (3.80), obtendo

$$
\left(J_{n}^{+}\right)^{\prime}\left(u_{n}, v_{n}\right)\left[v_{n}-P_{n} v, u_{n}-P_{n} u\right]=0, \quad \text { para todo } n>k+m .
$$

Uma vez que $u_{n}-P_{n} u, v_{n}-P_{n} v \rightarrow 0$ em $L^{2}$ e $u_{n}, v_{n}$ são limitadas em $L^{2}$, usando (1.2), 
temos as seguintes convergências:

$$
\left\{\begin{array}{l}
a \int_{\Omega} v_{n}\left(v_{n}-P_{n} v\right)+u_{n}\left(u_{n}-P_{n} u\right)+b \int_{\Omega} u_{n}\left(v_{n}-P_{n} v\right)+\int_{\Omega} v_{n}\left(u_{n}-P_{n} u\right) \rightarrow 0 \\
\int_{\Omega} f_{1}\left(x, v_{n}\right)\left(u_{n}-P_{n} u\right)+\int_{\Omega} f_{2}\left(x, u_{n}\right)\left(v_{n}-P_{n} v\right) \rightarrow 0 \\
\int_{\Omega} h_{1}\left(u_{n}-P_{n} u\right)+\int_{\Omega} h_{2}\left(v_{n}-P_{n} v\right) \rightarrow 0 .
\end{array}\right.
$$

Segue então de (3.84), que

$$
\int_{\Omega} \nabla v_{n} \nabla\left(v_{n}-P_{n} v\right)+\int_{\Omega} \nabla u_{n} \nabla\left(u_{n}-P_{n} u\right) \rightarrow 0
$$

que é equivalente a

$$
\int_{\Omega} \nabla v_{n} \nabla\left(v_{n}-v+v-P_{n} v\right)+\int_{\Omega} \nabla u_{n} \nabla\left(u_{n}-u+u-P_{n} u\right) \rightarrow 0
$$

e portanto

$$
\int_{\Omega}\left[\left|\nabla v_{n}\right|^{2}-\nabla v_{n} \nabla v+\left|\nabla u_{n}\right|^{2}-\nabla u_{n} \nabla u+\nabla v_{n} \nabla\left(v-P_{n} v\right)+\nabla u_{n} \nabla\left(u-P_{n} u\right)\right] \rightarrow 0 .
$$

Os dois últimos termos tendem a zero, pois $\left(P_{n} v, P_{n} u\right) \rightarrow(v, u)$ em $E$ e $\left(v_{n}, u_{n}\right)$ é limitada em E. Segue então que

$$
\int_{\Omega}\left|\nabla v_{n}\right|^{2}-\int_{\Omega} \nabla v_{n} \nabla v+\int_{\Omega}\left|\nabla u_{n}\right|^{2}-\int_{\Omega} \nabla u_{n} \nabla u \rightarrow 0
$$

isto é, $\left\|\left(u_{n}, v_{n}\right)\right\|_{E} \rightarrow\|(u, v)\|_{E}$, e portanto $\left(u_{n}, v_{n}\right) \rightarrow(u, v)$ fortemente em $E$.

Desta forma concluímos que $J^{+}\left(\mathbf{u}_{n}^{+}\right) \rightarrow J^{+}\left(\mathbf{u}^{+}\right)$e portanto $J^{+}\left(\mathbf{u}^{+}\right)=c^{+}$.

Pelo mesmo argumento, existe $\mathbf{v}^{+} \in E$, ponto crítico de $J^{+}$em $E$, tal que $\mathbf{v}_{n}^{+} \rightarrow \mathbf{v}^{+}$em $E$ e $J^{+}\left(\mathbf{v}^{+}\right)=d^{+}$. Além disso, $\mathbf{u}^{+} \neq \mathbf{v}^{+}$pois $J\left(\mathbf{u}^{+}\right)=c^{+} \leq E_{\delta}-1$ e $J\left(\mathbf{v}^{+}\right)=d^{+} \geq E_{\delta}$. Com isto, concluímos a demonstração do Teorema 3.1 .

O Teorema 3.2 é facilmente obtido por uma mudança de incógnitas.

Demonstração do Teorema 3.2. O resultado é consequência direta do Teorema 3.1. Seja 
$(u, v)$ uma das soluções de $(\underline{1.1}+)$ e denotemos $(u, \bar{v})=(u,-v)$. Assim

$$
\begin{cases}-\Delta u=a u-b \bar{v}+f_{1}(x,-\bar{v})+h_{1}(x) & \text { em } \Omega, \\ \Delta \bar{v}=b u-a \bar{v}+f_{2}(x, u)+h_{2}(x) & \text { em } \Omega\end{cases}
$$

e portanto

$$
\begin{cases}-\Delta u=a u-b \bar{v}-\left(-f_{1}(x,-\bar{v})\right)+h_{1}(x) & \text { em } \Omega \\ -\Delta \bar{v}=-b u+a \bar{v}-f_{2}(x, u)-h_{2}(x) & \text { em } \Omega\end{cases}
$$

isto é, $(u, \bar{v})$ é solução do problema (1.1-) com os novos coeficientes $\widetilde{a}=a$ e $\widetilde{b}=-b$, as novas não linearidades $\widetilde{f}_{1}(x, v)=-f_{1}(x,-v), \widetilde{f}_{2}(x, u)=f_{2}(x, u)$, as quais satisfazem (1.2)

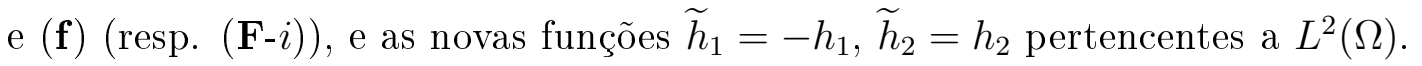

Se considerarmos a hipótese $(\mathbf{F})$, a condição $\int_{\Omega} h_{1} \phi+h_{2} \psi=0$ para $(\phi, \psi) \in Z$, do Teorema 3.1, torna-se $\int_{\Omega} \widetilde{h}_{1} \phi+\widetilde{h}_{2} \psi=0$ para $(\phi,-\psi) \in Z$, como no Teorema 3.2.

A prova do item $(d)$ segue de modo análogo do item $(b)$ do Teorema 3.1.

\subsection{Quase ressonância dupla}

Nesta seção apresentamos a demonstração dos resultados enunciados nos Teoremas 3.3 e 3.4, que lidam com dois casos de quase ressonância dupla, ou seja, quando os valores $a+b$ e $a-b$ estão perto de autovalores do operador Laplaciano, simultaneamente.

A prova seguirá as mesmas linhas que o item $(a)$ do Teorema 3.1 .

Fixemos $\lambda_{k}$ (de multiplicidade $m \in \mathbb{N}$, em particular seja $\lambda_{k}=\lambda_{k+m-1}$ ) e $\lambda_{l}$ (de multiplicidade $t \in \mathbb{N}$, em particular seja $\lambda_{l}=\lambda_{l+t-1}$ ) os autovalores enunciados no Teorema 3.3 .

Observemos que se $a-b \in\left(\lambda_{l}, \lambda_{l}+\varepsilon\right)$ e $a+b \in\left(\lambda_{k}-\varepsilon, \lambda_{k}\right)$, para algum $\varepsilon>0$, então $\mu_{k}=1-\frac{a+b}{\lambda_{k}}$ e $\mu_{-l}=\frac{a-b}{\lambda_{l}}-1$ satisfazem

$$
0<\mu_{k}, \mu_{-l}<\frac{\varepsilon}{\min \left\{\lambda_{k}, \lambda_{l}\right\}}
$$

Para reproduzir mais uma vez a geometria das Proposições 3.13 e 3.15, definamos os 
espaços $V, Z, W$ da seguinte maneira:

$$
\left\{\begin{array}{l}
V=\overline{\operatorname{span}\left\{\boldsymbol{\psi}_{i}: i \in \mathbb{Z}_{0}, \mu_{i}<0, \mu_{i} \neq \mu_{-l} \text { e } \mu_{i} \neq \mu_{k}\right\}}, \\
Z=\overline{\operatorname{span}\left\{\boldsymbol{\psi}_{i}: i \in \mathbb{Z}_{0}, \mu_{i}=\mu_{k} \text { ou } \mu_{i}=\mu_{-l}\right\}} \\
W=\overline{\operatorname{span}\left\{\boldsymbol{\psi}_{i}: i \in \mathbb{Z}_{0}, \mu_{i}>0, \mu_{i} \neq \mu_{-l} \text { e } \mu_{i} \neq \mu_{k}\right\}}
\end{array}\right.
$$

Descrevemos abaixo como deve ser feita a construção da geometria que garantirá a existência de níveis críticos análogos a (3.68) e (3.69), destacando as principais diferenças nas demonstrações. Começamos com um lema, semelhante ao Lema 3.10, que apresenta estimativas nos subespaços $V, Z$ e $W$.

Lema 3.23. Suponhamos $a \pm b \notin \sigma(-\Delta)$ e sejam fixados $\lambda_{k}$ o primeiro autovalor maior que $a+b$, de multiplicidade $m$, e $\lambda_{l}$ o último autovalor menor que $a-b$, de multiplicidade t. Então existe uma constante $\beta_{a, b}>0$, dependendo de a e de $b$, tal que

$$
\begin{gathered}
B(\mathbf{u}, \mathbf{u}) \leq-\beta_{a, b}\|\mathbf{u}\|_{E}^{2} \text {, para todo } \mathbf{u} \in V, \\
B(\mathbf{u}, \mathbf{u}) \geq \beta_{a, b}\|\mathbf{u}\|_{E}^{2}, \text { para todo } \mathbf{u} \in Z \oplus W .
\end{gathered}
$$

Além disso, se $a+b>0$ está suficientemente perto de $\lambda_{k}$ e $a-b$ de $\lambda_{l}$ (em particular, se $\operatorname{dist}\left(a+b, \sigma(-\Delta) \backslash\left\{\lambda_{k}\right\}\right)>\alpha>0$ e $\left.\operatorname{dist}\left(a-b, \sigma(-\Delta) \backslash\left\{\lambda_{l}\right\}\right)>\alpha>0\right)$, então existe $\eta_{\alpha}>0$, dependendo de $\alpha$, tal que

$$
\begin{aligned}
& B(\mathbf{u}, \mathbf{u}) \leq-\eta_{\alpha}\|\mathbf{u}\|_{E}^{2}, \text { para todo } \mathbf{u} \in V \\
& B(\mathbf{u}, \mathbf{u}) \geq \eta_{\alpha}\|\mathbf{u}\|_{E}^{2}, \text { para todo } \mathbf{u} \in W
\end{aligned}
$$

Observação 3.24. Como pode ser visto na demonstração abaixo, as constantes $\beta_{a, b}$ e $\eta_{\alpha}$ dependem também de $k$ e de $l$, que estamos considerando fixadas.

Demonstração. A prova segue pelo mesmo argumento do Lema 3.10, porém aqui $a-$ $b$ não está destacado do espectro $\sigma(-\Delta)$, e como consequência, a constante das duas primeiras estimativas depende também da diferença $a-b$. Em particular, podemos tomar $\beta_{a, b}=\inf \left\{\left|\mu_{i}\right|, i \in \mathbb{Z}_{0}\right\}$ (o ínfimo é positivo já que $\mu_{i} \rightarrow \pm 1$, para $i \rightarrow \pm \infty$ ) e $\eta_{\alpha}=$ $\left\{\inf \left|\mu_{i}\right|, i \in \mathbb{Z}_{V} \cup \mathbb{Z}_{W}\right\} \geq \frac{\alpha}{\max \left\{\lambda_{k+m}, \lambda_{l+t}\right\}}$. 
Novamente, para $\varepsilon$ suficientemente pequeno, o subespaço $Z$ definido em (3.92) coincide com o definido no enunciado no Teorema 3.3 .

Assim, com o Lema 3.23, obtemos as geometrias das proposições abaixo.

Proposição 3.25. Sob as hipóteses do Teorema 3.3, se $a \pm b \notin \sigma(-\Delta), \lambda_{k}$ é o primeiro autovalor maior que $a+b$ e $\lambda_{l}$ é o último menor que $a-b$, então existem $\Lambda_{a, b} \in \mathbb{R} e$ $\omega_{a, b}>0$, tais que

$$
\begin{array}{ll}
J^{+}(\mathbf{u}) \geq \Lambda_{a, b}, & \forall \mathbf{u} \in Z \oplus W, \\
J^{+}(\mathbf{u})<\Lambda_{a, b}, & \forall \mathbf{u} \in \omega S_{V}, \omega \geq \omega_{a, b} .
\end{array}
$$

Demonstração. A existência de $\Lambda_{a, b} \in \mathbb{R}$ satisfazendo (3.97), segue de (3.94) como na proposição [3.13, enquanto que a existência de $\omega_{a, b}>0$ satisfazendo (3.98), segue de (3.93).

Proposição 3.26. Sob as hipóteses do Teorema 3.3, suponhamos que $\lambda_{k}$ é o primeiro autovalor maior que $a+b$ e $\lambda_{l}$ é o último menor que $a-b$. Então existe $\varepsilon_{2}>0$, tal que para $a+b \in\left(\lambda_{k}-\varepsilon_{2}, \lambda_{k}\right)$ e $a-b \in\left(\lambda_{l}, \lambda_{l}+\varepsilon_{2}\right)$, existem $\Theta, \Lambda_{a, b} \in \mathbb{R}, \omega_{a, b}>r_{0}>0$, tais que valem (3.97), (3.98) e

$$
\begin{array}{rlrl}
J^{+}(\mathbf{u}) \geq \Theta, & & \forall \mathbf{u} \in W, \\
J^{+}(\mathbf{u})<\Theta-1, & \forall \mathbf{u} \in r_{0} S_{V Z}, \\
J^{+}(\mathbf{u})<\Theta-1, & \forall \mathbf{u} \in V \operatorname{com}\|\mathbf{u}\|_{E}>r_{0} .
\end{array}
$$

Demonstração. Seja

$$
\alpha=\frac{1}{2} \min \left\{\operatorname{dist}\left(\lambda_{k}, \sigma(-\Delta) \backslash\left\{\lambda_{k}\right\}\right), \operatorname{dist}\left(\lambda_{l}, \sigma(-\Delta) \backslash\left\{\lambda_{l}\right\}\right)\right\}
$$

e sejam $a+b \in\left(\lambda_{k}-\varepsilon_{2}, \lambda_{k}\right)$ e $a-b \in\left(\lambda_{l}, \lambda_{l}+\varepsilon_{2}\right)$, onde por enquanto pedimos apenas $\varepsilon_{2}<\alpha$, o que implica $\operatorname{dist}\left(a+b, \sigma(-\Delta) \backslash\left\{\lambda_{k}\right\}\right)>\alpha$ e $\operatorname{dist}\left(a-b, \sigma(-\Delta) \backslash\left\{\lambda_{l}\right\}\right)>\alpha$.

- A existência de $\Theta$ satisfazendo (3.99), segue diretamente de (3.96) como na Proposição 3.15. Uma vez que $\alpha$ é considerado fixado, sua dependência é omitida na notação da constante $\Theta$. 
- Seja $\mathbf{u} \in V$. Por (3.95) e (3.9+3.10),

$$
J^{+}(\mathbf{u}) \leq-\eta_{\alpha}\|\mathbf{u}\|_{E}^{2}+2 C_{0}\left(1+\|\mathbf{u}\|_{E}^{q}\right)+2 H\|\mathbf{u}\|_{E}
$$

Uma vez que $\eta_{\alpha}>0$ e $q \in(1,2)$ a estimativa (3.101) estará satisfeita desde que $r_{0}>\widetilde{r}$, onde $\widetilde{r}>0$ pode ser escolhido sem depender de $a$ e de $b$, dependendo apenas de $\alpha$.

- A fim de provar que existe $r_{0}>\widetilde{r}$ satisfazendo (3.100) para algum $\varepsilon_{2} \in(0, \alpha)$, procedendo como na Proposição 3.15, suponhamos por contradição que para quaisquer sequências $\varepsilon_{j} \rightarrow 0^{+}$e $\left\{r_{j}\right\}, \operatorname{com} r_{j}>\widetilde{r}$ para todo $j \in \mathbb{N}$, existam $\mathbf{u}_{j} \in r_{j} S_{V Z}$ e $a_{j}, b_{j} \in \mathbb{R}$, tais que $a_{j}+b_{j} \in\left(\lambda_{k}-\varepsilon_{j}, \lambda_{k}\right), a_{j}-b_{j} \in\left(\lambda_{l}, \lambda_{l}+\varepsilon_{j}\right)$ e $J_{a_{j}, b_{j}}^{+}\left(\mathbf{u}_{j}\right) \geq \Theta-1$.

Sem perda de generalidade, podemos supor que $r_{j} \rightarrow+\infty$ e que $\varepsilon_{j} r_{j}^{2} \rightarrow 0$. Escrevamos $\mathbf{u}_{j}=\mathbf{v}_{j}+\mathbf{z}_{j}$, onde $\mathbf{v}_{j} \in V$ e $\mathbf{z}_{j} \in Z$, para todo $j \in \mathbb{N}$. Por (3.91) e (3.95), temos

$$
B_{a_{j}, b_{j}}\left(\mathbf{u}_{j}, \mathbf{u}_{j}\right)=B_{a_{j}, b_{j}}\left(\mathbf{v}_{j}, \mathbf{v}_{j}\right)+B_{a_{j}, b_{j}}\left(\mathbf{z}_{j}, \mathbf{z}_{j}\right) \leq \frac{\varepsilon_{j}}{\min \left\{\lambda_{l}, \lambda_{k}\right\}}\left\|\mathbf{z}_{j}\right\|_{E}^{2}-\eta_{\alpha}\|\mathbf{u}\|_{E}^{2}
$$

Assim, para todo $j \in \mathbb{N}$, segue

$$
\Theta-1 \leq J^{+}\left(\mathbf{u}_{j}\right) \leq \frac{\varepsilon_{j}}{\min \left\{\lambda_{l}, \lambda_{k}\right\}}\left\|\mathbf{z}_{j}\right\|_{E}^{2}-\eta_{\alpha}\|\mathbf{u}\|_{E}^{2}-\mathcal{F}\left(\mathbf{u}_{j}\right)-\mathcal{H}\left(\mathbf{u}_{j}\right)
$$

Agora, prosseguindo como na prova da Proposição 3.15, obtemos uma contradição análoga à (3.59) ou à (3.66).

As constantes do enunciado da proposição, podem ser obtidas do seguinte modo: primeiramente tomamos $\Theta \in \mathbb{R}$ satisfazendo (3.99), então obtemos $\varepsilon_{2}>0$ e $r_{0}>0$ satisfazendo (3.100) e (3.101). Estas estimativas valem uniformemente para $a+b \in\left(\lambda_{k}-\varepsilon_{2}, \lambda_{k}\right)$ e $a-b \in\left(\lambda_{l}, \lambda_{l}+\varepsilon_{2}\right)$. Então, fixados $a, b$ respeitando estas condições, podemos obter a constante $\Lambda_{a, b} \in \mathbb{R}$ satisfazendo (3.97). Finalmente, tomamos $\omega_{a, b}>0$ satisfazendo (3.98); observemos que podemos escolher $\omega_{a, b}>r_{0}$, como desejado.

Com as geometrias obtidas acima e a estimativa (3.91) no lugar de (3.72), repetindo os mesmos argumentos das Seções 3.2 .2 e 3.2 .4 concluímos que o funcional $J^{+}$tem dois 
pontos críticos distintos em $E$, correspondendo a soluções fracas do problema $(1.1+)$, e então provando o Teorema 3.3 .

O Teorema 3.4 segue do Teorema 3.3 pela mesma mudança de incógnitas utilizada na demonstração do Teorema 3.2 .

\subsection{Condições (PS)}

Nesta seção apresentamos a prova dos Lemas 3.17 e 3.22, que mostram, respectivamente, que os funcionais $J_{n}^{ \pm}$satisfazem a condições (PS) no espaço finito dimensional $E_{n}$, para todo $n>k+m$, e que as sequências de pontos críticos obtidas nas Seções 3.2.2 e 3.2 .3 são limitadas.

Demonstração dos Lemas 3.17 e 3.22. Os dois lemas possuem enunciados diferentes, mas a demonstração é semelhante.

Pela hipótese $a \pm b \notin \sigma(-\Delta)$, os autovalores $\mu_{i}: i \in \mathbb{Z}_{0}$ são todos não nulos, logo podemos dividir o espaço $E$ nas componentes ortogonais

$$
E^{-}=\overline{\operatorname{span}\left\{\boldsymbol{\psi}_{i}: i \in \mathbb{Z}_{0}, \mu_{i}<0\right\}} \quad \text { e } \quad E^{+}=\overline{\operatorname{span}\left\{\boldsymbol{\psi}_{i}: i \in \mathbb{Z}_{0}, \mu_{i}>0\right\}},
$$

além disso, para cada $j>k+m$, sejam $E_{j}^{+}=E^{+} \cap E_{j}$ e $E_{j}^{-}=E^{-} \cap E_{j}$. Como $\mu_{i} \rightarrow \pm 1$ quando $i \rightarrow \pm \infty$, podemos definir $\xi:=\inf \left\{\left|\mu_{i}\right|:\left|\mu_{i}\right|>0, i \in \mathbb{Z}_{0}\right\}>0$ e vale

$$
\begin{cases}B(\mathbf{u}, \mathbf{u}) \leq-\xi\|\mathbf{u}\|_{E}^{2}, & \text { para todo } \mathbf{u} \in E^{-} \\ B(\mathbf{u}, \mathbf{u}) \geq \xi\|\mathbf{u}\|_{E}^{2}, & \text { para todo } \mathbf{u} \in E^{+}\end{cases}
$$

Claramente $\xi$ depende de $a, b$, mas isso é irrelevante aos fins desta demonstração.

Escrevamos $\mathbf{u}_{j}=\mathbf{v}_{j}+\mathbf{w}_{j}$ onde $\mathbf{v}_{j} \in E_{j}^{-}$e $\mathbf{w}_{j} \in E_{j}^{+}$. Mostremos que $\mathbf{v}_{j}$ e $\mathbf{w}_{j}$ são limitadas em $E$.

De fato, testando (3.67) (resp. (3.78) $) \operatorname{com} \phi=\mathbf{v}_{j}$, temos

$$
\left|B\left(\mathbf{u}_{j}, \mathbf{v}_{j}\right) \mp F^{\prime}\left(\mathbf{u}_{j}\right) \mathbf{v}_{j} \mp H^{\prime}\left(\mathbf{u}_{j}\right) \mathbf{v}_{j}\right| \leq \varepsilon_{j}\left\|\mathbf{v}_{j}\right\|_{E}
$$

Por (3.24), temos $B\left(\mathbf{u}_{j}, \mathbf{v}_{j}\right)=B\left(\mathbf{v}_{j}, \mathbf{v}_{j}\right), \operatorname{logo}$

$$
-\varepsilon_{j}\left\|\mathbf{v}_{j}\right\|_{E} \leq B\left(\mathbf{v}_{j}, \mathbf{v}_{j}\right)+S_{0}\left(1+\left\|\mathbf{u}_{j}\right\|_{E}^{q-1}\right)\left\|\mathbf{v}_{j}\right\|_{E}+H\left\|\mathbf{v}_{j}\right\|_{E} .
$$


Usando (3.105), segue

$$
-\varepsilon_{j}\left\|\mathbf{v}_{j}\right\|_{E} \leq-\xi\left\|\mathbf{v}_{j}\right\|_{E}^{2}+S_{0}\left(1+\left\|\mathbf{u}_{j}\right\|_{E}^{q-1}\right)\left\|\mathbf{v}_{j}\right\|_{E}+H\left\|\mathbf{v}_{j}\right\|_{E}
$$

implicando

$$
\xi\left\|\mathbf{v}_{j}\right\|_{E}^{2} \leq \varepsilon_{j}\left\|\mathbf{v}_{j}\right\|_{E}+S_{0}\left\|\mathbf{u}_{j}\right\|_{E}^{q-1}\left\|\mathbf{v}_{j}\right\|_{E}+\left(S_{0}+H\right)\left\|\mathbf{v}_{j}\right\|_{E},
$$

e dividindo por $\left\|\mathbf{v}_{j}\right\|_{E}$, temos

$$
\xi\left\|\mathbf{v}_{j}\right\|_{E} \leq \varepsilon_{j}+S_{0}\left\|\mathbf{u}_{j}\right\|_{E}^{q-1}+S_{0}+H
$$

Por outro lado, testando (3.67) (resp. (3.78) ) com $\phi=\mathbf{w}_{j}$ e procedendo de modo análogo, temos

$$
B\left(\mathbf{w}_{j}, \mathbf{w}_{j}\right)-S_{0}\left(1+\left\|\mathbf{u}_{j}\right\|_{E}^{q-1}\right)\left\|\mathbf{w}_{j}\right\|_{E}-H\left\|\mathbf{w}_{j}\right\|_{E} \leq \varepsilon_{j}\left\|\mathbf{w}_{j}\right\|_{E} .
$$

Por (3.105), vale

$$
\xi\left\|\mathbf{w}_{j}\right\|_{E}^{2}-S_{0}\left(1+\left\|\mathbf{u}_{j}\right\|_{E}^{q-1}\right)\left\|\mathbf{w}_{j}\right\|_{E}-H\left\|\mathbf{w}_{j}\right\|_{E} \leq \varepsilon_{j}\left\|\mathbf{w}_{j}\right\|_{E},
$$

e dividindo por $\left\|\mathbf{w}_{j}\right\|_{E}$, segue

$$
\xi\left\|\mathbf{w}_{j}\right\|_{E} \leq \varepsilon_{j}+S_{0}\left\|\mathbf{u}_{j}\right\|_{E}^{q-1}+S_{0}+H
$$

De (3.110) e (3.113), temos

$$
\xi\left\|\mathbf{u}_{j}\right\|_{E} \leq 2 \varepsilon_{j}+2 S_{0}\left\|\mathbf{u}_{j}\right\|_{E}^{q-1}+2\left(S_{0}+H\right)
$$

implicando que $\left\{\mathbf{u}_{j}\right\}$ é limitada em $E$, uma vez que $q-1<1$.

Isto conclui a demonstração do Lema 3.22, Para o Lema 3.17, uma vez que $\left\{\mathbf{u}_{j}\right\} \subseteq E_{n}$, que é finito dimensional, segue que existe uma subsequência convergente. 


\section{Capítulo}

\section{4}

\section{O caso ressonante}

Neste capítulo estudamos um sistema com parte linear ressonante. Neste caso, a parte quadrática do funcional não satisfaz a condição (PS) e é nula no autoespaço ressonante, o que significa que não teríamos a estimativa (3.29), que usamos para provar (3.39).

Para obtermos resultados de existência e multiplicidade de soluções, como os obtidos no Capítulo [3, precisamos de uma perturbação sublinear que tenha o duplo papel de recuperar a condição (PS) e a geometria de sela da Proposição 3.13, sem porém cancelar os efeitos de $f_{1}, f_{2}$, necessários para obter a segunda geometria de sela.

Em particular, consideremos os seguintes problemas $(4.1 \pm)$

$$
\begin{cases}-\Delta u=a u+b v+\eta g_{1}(x, v) \pm\left(f_{1}(x, v)+h_{1}(x)\right) & \text { em } \Omega \\ -\Delta v=b u+a v+\eta g_{2}(x, u) \pm\left(f_{2}(x, u)+h_{2}(x)\right) & \text { em } \Omega \\ u(x)=v(x)=0 & \text { em } \partial \Omega\end{cases}
$$

onde $\Omega$ é um aberto limitado de $\mathbb{R}^{N}, f_{1}, f_{2}$ satisfazem (1.2) e $g_{1}, g_{2}$ a seguinte condição

$$
\begin{aligned}
& g_{i}: \Omega \times \mathbb{R} \rightarrow \mathbb{R} \text { é função de Carathéodory para cada } i=1,2, \\
& \text { e existem constantes } c_{1}, c_{2}>0 \text { e } r \in(q, 2), \text { tais que } \\
& g_{i}(x, t) t \geq 0 \text { e } c_{1}\left(|t|^{r-1}-1\right) \leq\left|g_{i}(x, t)\right| \leq c_{2}\left(1+|t|^{r-1}\right) .
\end{aligned}
$$

Observemos que a condição $r \in(q, 2)$ implica que as funções $g_{1}, g_{2}$ são sublineares, mas de ordem maior, no infinito, com respeito às funções $f_{1}, f_{2}$. 
Com estas hipóteses, as mesmas técnicas utilizadas para demonstrar os Teoremas de 3.1 a 3.4 podem ser aplicadas, com pequenas adaptações, construindo as mesmas geometrias de sela e fazendo a aproximação finito dimensional para obter pontos críticos do funcional associado ao problema. Um pouco mais complexo será demonstrar as condições (PS), análogas às dos Lemas 3.17 e 3.22 .

Provaremos os seguintes resultados.

Teorema 4.1. Sejam $\lambda_{k}, \lambda_{l}$ autovalores do operador Laplaciano (não necessariamente distintos), $a, b \in \mathbb{R}$, tais que $a+b=\lambda_{k}$ e $\lambda_{l}$ é o primeiro autovalor acima de $a-b$, $e$

$$
Z=\operatorname{span}\left\{(\phi, \phi): \phi \in H_{\lambda_{k}}\right\}
$$

Se $f_{1}, f_{2}$ satisfazem (1.2) e (ff) (ou (프) ), $g_{1}, g_{2}$ satisfazem (4.2) e $h_{1}, h_{2} \in L^{r^{\prime}}(\Omega)$ (onde $\frac{1}{r}+\frac{1}{r^{\prime}}=1$ ), ent $\tilde{a} o$

(a) Dado $\delta>0$, tal que dist $(a-b, \sigma(-\Delta))>\delta$, existe $\varepsilon_{0}>0$ dependendo de $\delta$, tal que para $\eta \in\left(-\varepsilon_{0}, 0\right)$, existem duas soluções do problema $\left.\sqrt{4.1}+\right)$.

(b) Dado $\delta>0$, tal que dist $(a-b, \sigma(-\Delta))>\delta$, existe $\varepsilon_{1}>0$ dependendo de $\delta$, tal que para $\eta \in\left(0, \varepsilon_{1}\right)$, existem duas soluções do problema (4.1-).

Observação 4.2. Um exemplo de funções que satisfaçam as hipóteses do teorema, são $h_{1}=h_{2}=0, f_{1}, f_{2}$ como na Observação 3.5 e $g_{1}(x, u)=g_{2}(x, u)=|u|^{r-2} u$, com $q<r<$ 2 .

Como consequência do Teorema 4.1, é possível mostrar também um resultado de existência de soluções para o caso em que $a-b$ é ressonante enquanto $a+b$ está suficiente longe de $\sigma(-\Delta)$.

Teorema 4.3. Sejam $\lambda_{k}, \lambda_{l}$ autovalores do operador Laplaciano (não necessariamente distintos), $a, b \in \mathbb{R}$, tais que $a-b=\lambda_{k}$ e $\lambda_{l}$ é o primeiro autovalor acima de $a+b$, $e$

$$
Z=\operatorname{span}\left\{(\phi,-\phi): \phi \in H_{\lambda_{k}}\right\}
$$

Se $f_{1}, f_{2}$ satisfazem (1.2) e (ff) (ou (프) $), g_{1}, g_{2}$ satisfazem (4.2) e $h_{1}, h_{2} \in L^{r^{\prime}}(\Omega)$ (onde $\frac{1}{r}+\frac{1}{r^{\prime}}=1$ ), ent $\tilde{a} O$ 
(c) Dado $\delta>0$ tal que dist $(a+b, \sigma(-\Delta))>\delta$, existe $\varepsilon_{0}>0$ tal que para $\eta \in\left(0, \varepsilon_{0}\right)$, existem duas soluções do problema 4.1 -).

(d) Dado $\delta>0$ tal que dist $(a+b, \sigma(-\Delta))>\delta$, existe $\varepsilon_{1}>0$ tal que para $\eta \in\left(-\varepsilon_{1}, 0\right)$, existem duas soluções do problema $4.4+1$.

Como no Capítulo 3 é possível estender os teoremas acima para incluir a possibilidade de ressonância dupla.

Teorema 4.4. Sejam $\lambda_{k}, \lambda_{l}$ autovalores do operador Laplaciano (não necessariamente distintos), $a, b \in \mathbb{R}$, tais que $a+b=\lambda_{k}$ e $a-b=\lambda_{l}$, e

$$
Z=\operatorname{span}\left\{(\phi, \phi): \phi \in H_{\lambda_{k}}, \quad(\phi,-\phi): \phi \in H_{\lambda_{l}}\right\}
$$

Se $f_{1}, f_{2}$ satisfazem (1.2) e (ff) (ou (파) ), $g_{1}, g_{2}$ satisfazem (4.2) e $h_{1}, h_{2} \in L^{r^{\prime}}(\Omega)$ (onde $\frac{1}{r}+\frac{1}{r^{\prime}}=1$ ), então existe $\varepsilon_{2}>0$, tal que

(e) se $\eta \in\left(-\varepsilon_{2}, 0\right)$, então existem duas soluções do problema $\underline{4.1}+$ ),

(f) se $\eta \in\left(0, \varepsilon_{2}\right)$, então existem duas soluções do problema 4.1 -).

Como já dito, as demonstrações dos Teoremas 4.1, 4.3 e 4.4 seguem pelo mesmo argumento que utilizamos para demonstrar os resultados do Capítulo 3. Devido ao fato de $a+b$ (ou $a-b$ ) ser ressonante e à inserção dos termos $g_{1}$ e $g_{2}$ no sistema, teremos algumas diferenças na forma de obter as geometrias das Proposições 3.13 e 3.15, e principalmente na demonstração das condições (PS).

Apresentamos, na Seção 4.1, as principais diferenças nas demonstrações que levam à geometria de duas selas, enquanto na Seção 4.2 demonstramos as novas condições (PS).

\subsection{Duas geometrias de ponto de sela no caso ressonante}

Nesta seção apresentamos as principais diferenças para obter as duas geometrias de sela que permitirão demonstrar os Teoremas 4.1, 4.3 e 4.4.

Primeiramente precisamos fazer algumas considerações. Sejam os funcionais de classe $\mathcal{C}^{1}$ (ver apêndice $\mathrm{C}$ ), dados por

$$
J_{\eta}^{ \pm}(\mathbf{u})= \pm \frac{1}{2} B(\mathbf{u}, \mathbf{u}) \mp \eta \mathcal{G}(\mathbf{u})-\mathcal{F}(\mathbf{u})-\mathcal{H}(\mathbf{u})
$$


onde $B, \mathcal{F}, \mathcal{H}$ são os mesmos definidos na Seção 3.1 ,

$$
\mathcal{G}(\mathbf{u})=\int_{\Omega} G_{1}(x, v)+\int_{\Omega} G_{2}(x, u)
$$

e $G_{i}(x, t)=\int_{0}^{t} g_{i}(x, s) d s$, para $i=1,2$.

Estaremos assumindo nesta seção que os funcionais $J_{\eta}^{ \pm}$satisfaçam as condições (PS) dadas pelos Lemas 4.5 e 4.6, que serão demonstrados na próxima seção (análogos aos Lemas 3.22 e 3.17 da Seção 4.2).

Apresentamos a seguir a ideia da demonstração do item (a) do Teorema 4.1.

Precisaremos de estimativas um pouco mais fortes que (3.9, 3.10), sobre as aplicações $\mathcal{H}$ e $\mathcal{F}$, em particular, podemos mostrar (de modo análogo à prova do Lema 3.8) que existem constantes $S_{0}>0$ e $H>0$, tais que

$$
\begin{aligned}
|\mathcal{H}(\mathbf{u})| \leq H\|\mathbf{u}\|_{L^{r} \times L^{r}}, & \left|\mathcal{H}^{\prime}(\mathbf{u})[\boldsymbol{\phi}]\right| \leq H\|\phi\|_{L^{r} \times L^{r}}, \\
|\mathcal{F}(\mathbf{u})| \leq S_{0}\left(1+\|\mathbf{u}\|_{L^{q} \times L^{q}}^{q}\right), & \left|\mathcal{F}^{\prime}(\mathbf{u})[\boldsymbol{\phi}]\right| \leq S_{0}\left(1+\|\mathbf{u}\|_{\left[L^{2}\right]^{2}}^{q-1}\right)\|\boldsymbol{\phi}\|_{\left[L^{2}\right]^{2}} .
\end{aligned}
$$

De fato, (4.4) é consequência de $h_{1}, h_{2} \in L^{r^{\prime}}(\Omega)$ e (4.5) pode ser deduzida como (3.10) $(\operatorname{ver}(\underline{3.13}))$.

Quanto às funções $g_{1}, g_{2}$, notemos que por (4.2), segue

$$
G_{i}(x, t) \geq C_{1}\left(|t|^{r}-1\right) \quad \text { e } \quad\left|G_{i}(x, t)\right| \leq C_{2}\left(1+|t|^{r}\right),
$$

para todo $t \in \mathbb{R}$ e $i=1,2$. Desta forma, valem também para todo $\mathbf{u} \in E$

$$
\begin{gathered}
\mathcal{G}(\mathbf{u}) \geq \widetilde{C}_{1}\left(\|\mathbf{u}\|_{L^{r} \times L^{r}}^{r}-1\right), \\
|\mathcal{G}(\mathbf{u})| \leq \widetilde{C}_{2}\left(1+\|\mathbf{u}\|_{L^{r} \times L^{r}}^{r}\right) .
\end{gathered}
$$

Além disso, de $g_{i}(x, t) t \geq 0$, para todo $t \in \mathbb{R}$, temos

$$
G_{i}(x, u) \geq 0, \quad \forall u \in H
$$

Notemos que, definindo $V, Z$ e $W$ como em (3.26), não teremos as estimativas (3.28) e (3.29), uma vez que $a+b=\lambda_{k}$, mas ainda são válidas (3.30) e (3.31). Observemos também 
que, por (4.6) e (4.7), $\mathcal{G}(\mathbf{u})$ é de ordem menor que $\|\mathbf{u}\|_{E}^{2}$, porém maior que $\|\mathbf{u}\|_{L^{q} \times L^{q}}^{q}$, e este fato é o que nos permite ainda construir as geometrias das Proposições 3.13 e 3.15 .

A seguir comentamos as principais diferenças para obtermos as geometrias de sela para o funcional $J_{\eta}^{+}$, com $\eta<0$.

- Em (3.41), observemos que se $\mathbf{u}=\mathbf{z}+\mathbf{w}$, com $\mathbf{z} \in Z$ e $\mathbf{w} \in W$, temos

$$
B(\mathbf{u}, \mathbf{u})=B(\mathbf{z}, \mathbf{z})+B(\mathbf{w}, \mathbf{w})=B(\mathbf{w}, \mathbf{w})
$$

pois $B(\mathbf{z}, \mathbf{z})=0$, uma vez que $\mu_{k}=0$. Portanto por (3.31), (4.44.4.6), temos

$$
\begin{aligned}
J_{\eta}^{+}(\mathbf{u}) & \geq G_{\alpha, \delta}\|\mathbf{w}\|_{E}^{2}+(-\eta) \mathcal{G}(\mathbf{u})-S_{0}\left(1+\|\mathbf{u}\|_{L^{q} \times L^{q}}^{q}\right)-H\|\mathbf{u}\|_{L^{r} \times L^{r}} \\
& \geq(-\eta) \widetilde{C}_{1}\left(\|\mathbf{u}\|_{L^{r} \times L^{r}}^{r}-1\right)-S_{0}\left(1+C\|\mathbf{u}\|_{L^{r} \times L^{r}}^{q}\right)-H\|\mathbf{u}\|_{L^{r} \times L^{r}}
\end{aligned}
$$

e como $(-\eta)>0$ e $r>q>1$, deduzimos que existe $K_{\eta} \in \mathbb{R}$, tal que vale estimativa

$$
J_{\eta}^{+}(\mathbf{u}) \geq K_{\eta}, \quad \forall \mathbf{u} \in Z \oplus W,
$$

análoga a (3.39) .

- Em (3.42), procedemos da mesma forma para estimar as aplicações $B, \mathcal{F}$ e $\mathcal{H}$ (porém utilizando (3.30) no lugar de (3.28) para a aplicação $B$ ), e ainda, usamos (4.7) para estimar $\mathcal{G}$. Desta forma o termo com $\|\mathbf{u}\|_{E}^{2}$ continuará dominando, uma vez que $r<2$. Com isto obtemos que existe $\rho_{\eta, \delta}>0$, dependendo de $\eta$ e de $\delta$, tal que

$$
J_{\eta}^{+}(\mathbf{u})<K_{\eta}, \quad \forall \mathbf{u} \in \rho S_{V}, \rho \geq \rho_{\eta, \delta},
$$

resultado análogo ao obtido em (3.40).

Pelo mesmo raciocínio, em (3.46) e (3.47), obtemos que existem $\widetilde{R}>0$ (dependendo apenas de $\delta$ ) e $E_{\delta} \in \mathbb{R}$, tais que

$$
\begin{aligned}
J^{+}(\mathbf{u}) \geq E_{\delta}, & \forall \mathbf{u} \in W, \\
J^{+}(\mathbf{u})<E_{\delta}-1, & \forall \mathbf{u} \in V \operatorname{com}\|\mathbf{u}\|_{E}>\widetilde{R} .
\end{aligned}
$$

Estas correspondem às estimativas (3.43) e (3.45). 
- Por fim, no lugar de (3.44), mostramos que existem $R_{\delta}>\widetilde{R}$ e $\eta_{0}>0$, tais que

$$
J_{\eta}^{+}(\mathbf{u})<E_{\delta}-1, \quad \forall \mathbf{u} \in R_{\delta} S_{V Z},
$$

e $\eta \in\left(-\eta_{0}, 0\right)$. Para provar esta afirmação, notemos que $J_{\eta_{2}}^{+} \leq J_{\eta_{1}}^{+}$, se $\eta_{1}<\eta_{2}$, portanto é suficiente mostrarmos que existe um $\eta<0$ que satisfaça a afirmação.

A prova é feita por contradição, supondo que existam sequências $\eta_{j} \rightarrow 0^{-}$e $R_{j} \rightarrow$ $+\infty$, tais que para todo $j \in \mathbb{N}$, existe $\mathbf{u}_{j} \in R_{j} S_{V Z}$, tal que $J_{\eta_{j}}^{+}\left(\mathbf{u}_{j}\right) \geq E_{\delta}-1$. Sem perda de generalidade, suponhamos $\eta_{j} R_{j}^{r} \rightarrow 0$. Escrevendo $\mathbf{u}_{j}=\mathbf{v}_{j}+\mathbf{z}_{j}, \operatorname{com} \mathbf{v}_{j} \in V$ e $\mathbf{z}_{j} \in Z$, por (3.30) e (4.7), temos

$$
\begin{aligned}
& E_{\delta}- \leq J_{\eta_{j}}^{+}\left(\mathbf{u}_{j}\right) \leq-G_{\alpha, \delta}\left\|\mathbf{v}_{j}\right\|_{E}^{2}+\left(-\eta_{j}\right) C\left(1+\left\|\mathbf{u}_{j}\right\|_{E}^{r}\right)-\mathcal{F}\left(\mathbf{u}_{j}\right)-\mathcal{H}\left(\mathbf{u}_{j}\right) \\
& \leq-G_{\alpha, \delta}\left\|\mathbf{v}_{j}\right\|_{E}^{2}+\left(-\eta_{j}\right) C\left[\left(\left\|\mathbf{z}_{j}\right\|_{E}^{2}+\left\|\mathbf{v}_{j}\right\|_{E}^{2}\right)^{r / 2}+1\right]-\mathcal{F}\left(\mathbf{u}_{j}\right)-\mathcal{H}\left(\mathbf{u}_{j}\right) .
\end{aligned}
$$

Agora basta dividir (4.14) por $R_{j}^{2}$ e proceder como feito na Proposição 3.15 para, no caso da hipótese (f) , chegar ao análogo de (3.59), que neste caso teria a forma

$$
G_{\alpha, \delta} R_{j}^{2}\left\|\widehat{\mathbf{v}}_{j}\right\|_{E}^{2}+R_{j} \leq-C \eta_{j} R_{j}^{r}+2 D_{M}-E_{\delta}+1
$$

o que é de novo uma contradição, pois como $-\eta_{j} R_{j}^{r} \rightarrow 0$, quando $j \rightarrow \infty$, o lado direito da desigualdade permanece limitado, enquanto o lado esquerdo $G_{\alpha, \delta} R_{j}^{2}\left\|\widehat{\mathbf{v}}_{j}\right\|_{E}^{2}+$ $R_{j} \rightarrow+\infty$, quando $j \rightarrow \infty$, uma vez que $R_{j} \rightarrow \infty$. No caso da hipótese $(\mathbf{F})$ o resultado é obtido da mesma forma.

Utilizando as geometrias descritas acima e seguindo os mesmos passos das Seções 3.2 .2 e 3.2.4, podemos mostrar a existência de dois pontos críticos distintos do funcional $J_{\eta}^{+}$, e portanto soluções para o problema (4.1+). Isso demonstra o item $(a)$ do Teorema 4.1 enquanto que o item $(b)$ segue pelo mesmo argumento utilizado na Seção 3.2 .3 ,

Novamente o Teorema 4.3 segue por uma simples mudança de incógnitas.

Demonstração do Teorema 4.3. O resultado é consequência direta do Teorema 4.1, Seja $(u, v)$ uma das soluções de (4.1+) e denotemos $(u, \bar{v})=(u,-v)$. Assim

$$
\begin{cases}-\Delta u=a u-b \bar{v}-\eta\left(-g_{1}(x,-\bar{v})\right)-\left(-f_{1}(x,-\bar{v})\right)+h_{1}(x) & \text { em } \Omega \\ -\Delta \bar{v}=-b u+a \bar{v}-\eta g_{2}(x, u)-f_{2}(x, u)-h_{2}(x) & \text { em } \Omega\end{cases}
$$


isto é, $(u, \bar{v})$ é solução do problema (4.1-) com os novos coeficientes $\widetilde{a}=a, \widetilde{b}=-b$ e $\widetilde{\eta}=-\eta$, as novas não linearidades

$$
\begin{cases}\widetilde{g}_{1}(x, v)=-g_{1}(x,-v), & \widetilde{g}_{2}(x, u)=g_{2}(x, u), \\ \widetilde{f}_{1}(x, v)=-f_{1}(x,-v), & \widetilde{f}_{2}(x, u)=f_{2}(x, u)\end{cases}
$$

e as novas funções $\widetilde{h}_{1}=-h_{1}, \widetilde{h}_{2}=h_{2}$. Como argumentado na prova do Teorema 3.2,

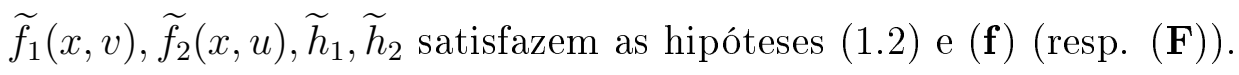

Além disso, notemos também que $\widetilde{g}_{1}$ e $\widetilde{g}_{2}$ também satisfazem (4.2).

A prova do item $(d)$ segue de modo análogo do item $(b)$ do Teorema 4.1 .

Demonstração do Teorema 4.4. Segue construindo as mesmas geometrias feitas nas demonstrações dos Teoremas 3.3 e 3.4, utilizando as adaptações descritas na prova do Teorema 4.1 ,

\subsection{Condições (PS) no caso ressonante}

Nesta seção demonstramos dois lemas análogos às condições (PS) da Seção 3.4 .

Lema 4.5. Sejam $\eta \neq 0, a, b \in \mathbb{R}, f_{1}, f_{2}$ satisfazendo (1.2), $g_{1}, g_{2}$ satisfazendo (4.2) $e$ $h_{1}, h_{2} \in L^{r^{\prime}}$. Se $a+b \in \sigma(-\Delta)$ e/ou $a-b \in \sigma(-\Delta)$, então toda sequência $\left\{\mathbf{u}_{j}\right\} \subset E$, tal que $\mathbf{u}_{j} \in E_{j}$, satisfazendo

$$
\left|\left(\left.J_{\eta}^{ \pm}\right|_{E_{j}}\right)^{\prime}\left(\mathbf{u}_{j}\right)[\phi]\right| \leq \varepsilon_{j}\|\phi\|_{E}, \quad \forall \phi \in E_{j},
$$

onde $\varepsilon_{j} \rightarrow 0$ quando $j \rightarrow+\infty$, é limitada em $E$.

Demonstração. Definamos o subespaço de $E$

$$
E^{0}=\overline{\operatorname{span}\left\{\boldsymbol{\psi}_{i}: i \in \mathbb{Z}_{0}, \mu_{i}=0\right\}}
$$

Para cada $j$ suficientemente grande, sejam $E_{j}^{0}=E^{0} \cap E_{j}, E_{j}^{+}=E^{+} \cap E_{j}$ e $E_{j}^{-}=E^{-} \cap E_{j}$, onde $E^{+}, E^{-}$foram definidos na Seção 3.4. Assim $E_{j}=E_{j}^{+} \oplus E_{j}^{0} \oplus E_{j}^{-}$.

Seja $\left\{\mathbf{u}_{j}\right\}$ uma sequência em $E$, com $\mathbf{u}_{j} \in E_{j}$. Escrevamos $\mathbf{u}_{j}=\mathbf{v}_{j}+\mathbf{w}_{j}+\mathbf{z}_{j}$, onde $\mathbf{v}_{j} \in E_{j}^{-}, \mathbf{w}_{j} \in E_{j}^{+}$e $\mathbf{z}_{j} \in E_{j}^{0}$. 
Testando (4.17) $\operatorname{com} \phi=\mathbf{v}_{j}$, por (3.105), (4.2) e (4.4.4.5), temos (veja (3.110))

$$
\xi\left\|\mathbf{v}_{j}\right\|_{E} \leq \varepsilon_{j}+|\eta| C\left(\left\|\mathbf{u}_{j}\right\|_{E}^{r-1}+1\right)+S_{0}\left(\left\|\mathbf{u}_{j}\right\|_{E}^{q-1}+1\right)+H
$$

Por outro lado, testando $\operatorname{com} \phi=\mathbf{w}_{i}$, segue (veja (3.113) )

$$
\xi\left\|\mathbf{w}_{j}\right\|_{E} \leq \varepsilon_{j}+|\eta| C\left(\left\|\mathbf{u}_{j}\right\|_{E}^{r-1}+1\right)+S_{0}\left(\left\|\mathbf{u}_{j}\right\|_{E}^{q-1}+1\right)+H
$$

Agora, suponhamos por contradição que $\left\|\mathbf{u}_{j}\right\|_{E} \rightarrow+\infty$, quando $j \rightarrow \infty$. Temos por (4.18) e (4.19), que

$$
\frac{\left\|\mathbf{v}_{j}\right\|_{E}}{\left\|\mathbf{u}_{j}\right\|_{E}}, \frac{\left\|\mathbf{w}_{j}\right\|_{E}}{\left\|\mathbf{u}_{j}\right\|_{E}} \rightarrow 0, \quad i \rightarrow \infty
$$

e portanto

$$
\frac{\left\|\mathbf{v}_{j}+\mathbf{w}_{j}\right\|_{E}}{\left\|\mathbf{u}_{j}\right\|_{E}} \rightarrow 0, \quad i \rightarrow \infty
$$

Uma vez que $\left\|\mathbf{z}_{j}\right\|_{E}^{2}=\left\|\mathbf{u}_{j}\right\|_{E}^{2}-\left\|\mathbf{v}_{j}+\mathbf{w}_{j}\right\|_{E}^{2}$, por (4.21), segue

$$
\frac{\left\|\mathbf{z}_{j}\right\|_{E}^{2}}{\left\|\mathbf{u}_{j}\right\|_{E}^{2}}=1-\frac{\left\|\mathbf{v}_{j}+\mathbf{w}_{j}\right\|_{E}^{2}}{\left\|\mathbf{u}_{j}\right\|_{E}^{2}} \rightarrow 1
$$

Então existe $\mathbf{z}_{0} \in E^{0}$, tal que passando a uma subsequência,

$$
\frac{\mathbf{z}_{j}}{\left\|\mathbf{u}_{j}\right\|_{E}} \rightarrow \mathbf{z}_{0}, \quad \text { em } E
$$

uma vez que $E^{0}$ é finito dimensional. Logo

$$
\frac{\mathbf{u}_{j}}{\left\|\mathbf{u}_{j}\right\|_{E}} \rightarrow \mathbf{z}_{0}, \quad \text { em } E
$$

Escrevendo $\mathbf{u}=\left(P_{1} \mathbf{u}, P_{2} \mathbf{u}\right)$ para todo $\mathbf{u} \in E$, temos para $i=1,2$

$$
\frac{P_{i} \mathbf{u}_{j}}{\left\|\mathbf{u}_{j}\right\|_{E}} \rightarrow P_{i} \mathbf{z}_{0}, \quad \text { em } H_{0}^{1}(\Omega) .
$$

Suponhamos inicialmente que $P_{1} \mathbf{z}_{0}, P_{2} \mathbf{z}_{0} \neq 0$. Assim, existem $\rho>1$ e $j_{0}$ suficientemente grande, tais que

$$
\frac{1}{\rho}\left\|P_{1} \mathbf{u}_{j}\right\|_{H}^{2} \leq\left\|P_{2} \mathbf{u}_{j}\right\|_{H}^{2} \leq \rho\left\|P_{1} \mathbf{u}_{j}\right\|_{H}^{2}, \quad j \geq j_{0}
$$


Por outro lado, por (4.21), temos

$$
\frac{\left\|P_{i}\left(\mathbf{v}_{j}+\mathbf{w}_{j}\right)\right\|_{H}}{\left\|\mathbf{u}_{j}\right\|_{H}} \rightarrow 0, \quad j \rightarrow \infty .
$$

Logo, dado $\alpha \in(0,1)$, existe um índice $j_{1}$ suficientemente grande (maior que $j_{0}$ ), tal que

$$
\left\|P_{i}\left(\mathbf{v}_{j}+\mathbf{w}_{j}\right)\right\|_{H}^{2} \leq \frac{\alpha^{2}}{\rho+1}\left\|\mathbf{u}_{j}\right\|_{E}^{2}=\frac{\alpha^{2}}{\rho+1}\left(\left\|P_{1} \mathbf{u}_{j}\right\|_{H}^{2}+\left\|P_{2} \mathbf{u}_{j}\right\|_{H}^{2}\right), \quad j \geq j_{1}
$$

e combinando com (4.26), temos

$$
\left\|P_{i}\left(\mathbf{v}_{j}+\mathbf{w}_{j}\right)\right\|_{H} \leq \alpha\left\|P_{i} \mathbf{u}_{j}\right\|_{H}, \quad j \geq j_{1}, \quad i=1,2 .
$$

Isso nos permite aplicar o Lema 4.7 (demonstrado abaixo), obtendo $\beta>0$ e $\kappa \in \mathbb{R}$, tais que

$$
\begin{aligned}
& \int_{\Omega}\left[g_{1}\left(x, P_{2} \mathbf{u}_{j}\right) P_{2} \mathbf{z}_{j}+g_{2}\left(x, P_{1} \mathbf{u}_{j}\right) P_{1} \mathbf{z}_{j}\right] \geq \\
& \quad \beta\left(\left\|P_{2} \mathbf{u}_{j}\right\|_{H}^{r}+\left\|P_{1} \mathbf{u}_{j}\right\|_{H}^{r}\right)-\kappa\left(\left\|P_{2} \mathbf{u}_{j}\right\|_{H}+\left\|P_{1} \mathbf{u}_{j}\right\|_{H}\right) \geq \beta C\left\|\mathbf{u}_{j}\right\|_{E}^{r}-\kappa C\left\|\mathbf{u}_{j}\right\|_{E}
\end{aligned}
$$

onde $C$ é uma constante positiva.

Assim, testando (4.17) $\operatorname{com} \phi=\frac{\mathbf{z}_{j}}{\left\|\mathbf{u}_{j}\right\|_{E}^{r}}$, e usando que $B\left(\mathbf{u}_{j}, \mathbf{z}_{j}\right)=0$, pois $\mathbf{z}_{j} \in E^{0}$, temos

$$
\begin{aligned}
&|\eta| \mid \int_{\Omega} g_{1}\left(x, P_{2} \mathbf{u}_{j}\right) \frac{P_{2} \mathbf{z}_{j}}{\left\|\mathbf{u}_{j}\right\|_{E}^{r}}+\int_{\Omega} g_{2}\left(x, P_{1} \mathbf{u}_{j}\right) \frac{P_{1} \mathbf{z}_{j}}{\left\|\mathbf{u}_{j}\right\|_{E}^{r}} \mid \\
&-\left|\mathcal{F}^{\prime}\left(\mathbf{u}_{j}\right) \frac{\mathbf{z}_{j}}{\left\|\mathbf{u}_{j}\right\|_{E}^{r}}+\mathcal{H}^{\prime}\left(\mathbf{u}_{j}\right) \frac{\mathbf{z}_{j}}{\left\|\mathbf{u}_{j}\right\|_{E}^{r}}\right| \leq \varepsilon_{j}\left\|\frac{\mathbf{z}_{j}}{\left\|\mathbf{u}_{j}\right\|_{E}^{r}}\right\|_{E} .
\end{aligned}
$$

Logo, por (4.30), temos

$$
|\eta|\left[\beta C \frac{\left\|\mathbf{u}_{j}\right\|_{E}^{r}}{\left\|\mathbf{u}_{j}\right\|_{E}^{r}}-\kappa C \frac{\left\|\mathbf{u}_{j}\right\|_{E}}{\left\|\mathbf{u}_{j}\right\|_{E}^{r}}\right]-\left|\mathcal{F}^{\prime}\left(\mathbf{u}_{j}\right) \frac{\mathbf{z}_{j}}{\left\|\mathbf{u}_{j}\right\|_{E}^{r}}+\mathcal{H}^{\prime}\left(\mathbf{u}_{j}\right) \frac{\mathbf{z}_{j}}{\left\|\mathbf{u}_{j}\right\|_{E}^{r}}\right| \leq \varepsilon_{j}\left\|\frac{\mathbf{z}_{j}}{\left\|\mathbf{u}_{j}\right\|_{E}^{r}}\right\|_{E}
$$

Uma vez que $r>q>1$, por (4.4-4.5), temos

$$
\left|\mathcal{F}^{\prime}\left(\mathbf{u}_{j}\right) \frac{\mathbf{z}_{j}}{\left\|\mathbf{u}_{j}\right\|_{E}^{r}}+\mathcal{H}^{\prime}\left(\mathbf{u}_{j}\right) \frac{\mathbf{z}_{j}}{\left\|\mathbf{u}_{j}\right\|_{E}^{r}}\right| \rightarrow 0, \quad j \rightarrow \infty
$$


Assim, fazendo $j \rightarrow \infty$ em (4.32), obtemos $|\eta| \beta C \leq 0$, implicando que $\beta \leq 0$, o que é uma contradição.

Suponhamos agora que $\mathbf{z}_{0}$ tenha uma componente nula: sem perda de generalidade seja $\mathbf{z}_{0}=\left(P_{1} \mathbf{z}_{0}, 0\right)$. Neste caso no lugar de (4.26), temos

$$
\left\|P_{2} \mathbf{u}_{j}\right\|_{H}^{2} \leq \rho\left\|P_{1} \mathbf{u}_{j}\right\|_{H}^{2}, \quad j \geq j_{0} .
$$

Assim obtemos (4.29) apenas para $i=1$. E no lugar de (4.30), podemos escrever

$$
\int_{\Omega} g_{2}\left(x, P_{1} \mathbf{u}_{j}\right) P_{1} \mathbf{z}_{j} \geq \beta\left\|P_{1} \mathbf{u}_{j}\right\|_{H}^{r}-\kappa\left\|P_{1} \mathbf{u}_{j}\right\|_{H}
$$

Logo, testando (4.17) $\operatorname{com} \phi=\frac{\mathbf{z}_{j}}{\left\|\mathbf{u}_{j}\right\|_{E}^{r}}$, temos

$$
\begin{aligned}
& \frac{|\eta|}{\left\|\mathbf{u}_{j}\right\|_{E}^{r}}\left[\beta\left\|P_{1} \mathbf{u}_{j}\right\|_{H}^{r}-\kappa\left\|P_{1} \mathbf{u}_{j}\right\|_{H}\right]- \\
& \left|\int_{\Omega} g_{1}\left(x, P_{2} \mathbf{u}_{j}\right) \frac{P_{2} \mathbf{z}_{j}}{\left\|\mathbf{u}_{j}\right\|_{E}^{r}}+\mathcal{F}^{\prime}\left(\mathbf{u}_{j}\right) \frac{\mathbf{z}_{j}}{\left\|\mathbf{u}_{j}\right\|_{E}^{r}}+\mathcal{H}^{\prime}\left(\mathbf{u}_{j}\right) \frac{\mathbf{z}_{j}}{\left\|\mathbf{u}_{j}\right\|_{E}^{r}}\right| \leq \varepsilon_{j}\left\|\frac{\mathbf{z}_{j}}{\left\|\mathbf{u}_{j}\right\|_{E}^{r}}\right\|_{E}
\end{aligned}
$$

Notemos que, utilizando (4.2) (raciocinando como em (3.15)), segue

$$
\left|\int_{\Omega} g_{1}\left(x, P_{2} \mathbf{u}_{j}\right) P_{2} \mathbf{z}_{j}\right| \leq C\left(\left\|P_{2} \mathbf{z}_{j}\right\|_{H}+\left\|P_{2} \mathbf{u}_{j}\right\|_{H}^{r-1}\left\|P_{2} \mathbf{z}_{j}\right\|_{H}\right),
$$

e portanto

$$
\int_{\Omega} g_{1}\left(x, P_{2} \mathbf{u}_{j}\right) \frac{P_{2} \mathbf{z}_{j}}{\left\|\mathbf{u}_{j}\right\|_{E}^{r}} \leq C\left(\frac{\left\|P_{2} \mathbf{z}_{j}\right\|_{H}}{\left\|\mathbf{u}_{j}\right\|_{H}^{r}}+\frac{\left\|P_{2} \mathbf{u}_{j}\right\|_{H}^{r-1}}{\left\|\mathbf{u}_{j}\right\|_{H}^{r-1}} \frac{\left\|P_{2} \mathbf{z}_{j}\right\|_{H}}{\left\|\mathbf{u}_{j}\right\|_{H}}\right) .
$$

Assim, usando $\frac{\left\|P_{2} \mathbf{z}_{j}\right\|_{H}}{\left\|\mathbf{u}_{j}\right\|_{E}} \rightarrow 0$ e $\frac{\left\|P_{2} \mathbf{u}_{j}\right\|_{H}}{\left\|\mathbf{u}_{j}\right\|_{E}} \rightarrow 0$ (veja (4.25)), temos

$$
\int_{\Omega} g_{1}\left(x, P_{2} \mathbf{u}_{j}\right) \frac{P_{2} \mathbf{z}_{j}}{\left\|\mathbf{u}_{j}\right\|_{E}^{r}} \rightarrow 0 \quad \text { e } \quad \frac{\left\|P_{1} \mathbf{u}_{j}\right\|_{H}}{\left\|\mathbf{u}_{j}\right\|_{E}} \rightarrow 1 .
$$

Portanto, fazendo $j \rightarrow+\infty$ em (4.35), segue novamente a contradição que $\beta \leq 0$.

Concluímos que a sequência $\left\{\mathbf{u}_{j}\right\}$ é limitada em $E$.

Lema 4.6. Sejam $\eta \neq 0, a, b \in \mathbb{R}, f_{1}, f_{2}$ satisfazendo (1.2), $g_{1}, g_{2}$ satisfazendo (4.2) $e$ $h_{1}, h_{2} \in L^{r^{\prime}}$. Fixando $n$ suficientemente grande, se $a+b \in \sigma(-\Delta)$ e/ou $a-b \in \sigma(-\Delta)$, 
então toda sequência $\left\{\mathbf{u}_{j}\right\} \subset E_{n}$, tal que

$$
\left|\left(\left.J_{\eta}^{ \pm}\right|_{E_{n}}\right)^{\prime}\left(\mathbf{u}_{j}\right)[\boldsymbol{\phi}]\right| \leq \varepsilon_{j}\|\phi\|_{E}, \quad \text { para todo } \phi \in E_{n}
$$

onde $\varepsilon_{j} \rightarrow 0$, quando $j \rightarrow \infty$, possui uma subsequência convergente em $E_{n}$.

Demonstração. Supondo que a sequência $\left\{\mathbf{u}_{j}\right\} \subset E_{n}$, para $n$ suficientemente grande, vale a mesma demonstração do Lema 4.5, se tomarmos $\mathbf{w}_{j} \in E_{n}^{+}$e $\mathbf{v}_{j} \in E_{n}^{-}$, mostrando que a sequência $\left\{\mathbf{u}_{j}\right\}$ é limitada. Como $E_{n}$ é finito dimensional, existe uma subsequência convergente.

\subsection{Estimativa da perturbação sublinear}

Na demonstração dos Lemas 4.5 e 4.6, utilizamos o Lema 4.7 que enunciamos e demonstramos abaixo. Para isso consideremos as seguintes afirmações, cujas demonstrações podem ser encontradas em [BBF83].

Seja $Y \subset H_{0}^{1}(\Omega)$ um subespaço gerado por um número finito de funções não nulas q. t. p. de $\Omega$.

(1) Dado $\sigma>0$, existe $a_{\sigma}>0$, tal que

$$
\left|\left\{x \in \Omega:|u(x)| \leq a_{\sigma}\|u\|_{H}\right\}\right|>|\Omega|-\sigma, \quad \forall u \in H_{0}^{1}(\Omega) .
$$

(2) Dado $\tau>0$, existe $b_{\tau}>0$, tal que

$$
\left|\left\{x \in \Omega:|z(x)| \geq b_{\tau}\|z\|_{H}\right\}\right|>|\Omega|-\tau, \quad \forall z \in Y \backslash\{0\}
$$

Lema 4.7 (do Lema 2.1 de [ST01]). Se g é uma função de Caratheódory satisfazendo (4.2), então existem $\beta>0, \alpha \in(0,1)$ e $\kappa \in \mathbb{R}$, tais que

$$
\int_{\Omega} g(x, u) z \geq \beta\|u\|_{H}^{r}-\kappa\|u\|_{H}
$$

para todo $u=z+w$, onde $z \in Y e\|w\|_{H} \leq \alpha\|u\|_{H}$. 
Demonstração. Sejam fixados, $\tau, \sigma>0$ tais que

$$
\tau+\sigma<|\Omega| / 2
$$

Para $\alpha \in(0,1 / 2)$, definamos o conjunto

$$
\mathcal{C}(\alpha)=\left\{u=z+w \in H_{0}^{1}(\Omega): z \in Y, \quad\|w\|_{H} \leq \alpha\|u\|_{H}\right\} .
$$

Assim para qualquer $u=z+w \in \mathcal{C}(\alpha)$, temos

$$
\|w\|_{H} \leq \alpha\|u\|_{H} \quad \text { e } \quad\|z\|_{H} \geq\|u\|_{H}-\|w\|_{H} \geq\|u\|_{H}-\alpha\|u\|_{H}>\frac{1}{2}\|u\|_{H} .
$$

Denotemos então os conjuntos

$$
\begin{gathered}
\Omega_{\sigma}^{w}=\left\{x \in \Omega:|w(x)| \leq a_{\sigma}\|w\|_{H}\right\}, \\
\Omega_{\tau}^{z}=\left\{x \in \Omega:|z(x)| \geq b_{\tau}\|z\|_{H}\right\},
\end{gathered}
$$

e consideremos o subconjunto de $\Omega$

$$
\Lambda=\Omega_{\sigma}^{w} \cap \Omega_{\tau}^{z}
$$

onde por (4.37) e (4.38) temos $|\Lambda|>|\Omega|-\tau-\sigma>|\Omega| / 2$.

Então para $x \in \Lambda$, temos pelas definições dos conjuntos $\Omega_{\sigma}^{w}, \Omega_{\tau}^{z}$ e por (4.40),

$$
|u(x)| \geq|z(x)|-|w(x)| \geq b_{\tau}\|z\|_{H}-a_{\sigma}\|w\|_{H} \geq \frac{b_{\tau}}{2}\|u\|_{H}-a_{\sigma} \alpha\|u\|_{H} .
$$

Separemos

$$
\int_{\Omega} g(x, u) z=\int_{\Omega} g(x, u) u-\int_{\Omega} g(x, u) w,
$$

onde, por (4.2), (4.40) e (4.41), temos

$$
\begin{aligned}
\int_{\Omega} g(x, u) u & \geq \int_{\Lambda} g(x, u) u \geq \int_{\Lambda} c_{1}\left(|u|^{r-1}-1\right)|u| \\
& \geq \frac{c_{1}|\Omega|}{2}\left(\frac{b_{\tau}}{2}-a_{\sigma} \alpha\right)^{r}\|u\|_{H}^{r}-C\|u\|_{H}
\end{aligned}
$$


onde $C$ denota (aqui e abaixo) uma constante que dependerá apenas de $\Omega, c_{1}, c_{2}, r$.

Por outro lado, utilizamos (4.2) e (4.40), obtendo

$$
\begin{aligned}
-\int_{\Omega} g(x, u) w \geq-\int_{\Omega}|g(x, u) w| & \geq-\int_{\Omega} c_{2}\left(1+|u|^{r-1}\right)|w| \\
& \geq-C \alpha\|u\|_{H}-C \alpha\|u\|_{H}^{r} .
\end{aligned}
$$

$\mathrm{Na}$ estimativa acima, usamos a seguinte desigualdade proveniente da Desigualdade de Hölder e de (4.40):

$$
\begin{aligned}
\int_{\Omega}|u|^{r-1}|w| & \leq\left(\int_{\Omega}|u|^{r-1 \frac{r}{r-1}}\right)^{\frac{r-1}{r}}\left(\int_{\Omega}|w|^{r}\right)^{\frac{1}{r}} \\
& =\|u\|_{L^{r}}^{r-1}\|w\|_{L^{r}} \leq C\|u\|_{H}^{r-1}\|w\|_{H} \leq C \alpha\|u\|_{H}^{r} .
\end{aligned}
$$

Portanto, por (4.42 4.44), temos

$$
\int_{\Omega} g(x, u) z \geq\left[\frac{c_{1}|\Omega|}{2}\left(\frac{b_{\tau}}{2}-a_{\sigma} \alpha\right)^{r}-C \alpha\right]\|u\|_{H}^{r}-C(1+\alpha)\|u\|_{H} .
$$

A este ponto, para $\alpha>0$ suficientemente pequeno, o coeficiente de $\|u\|_{H}^{r}$ torna-se positivo e isso conclui a demonstração. 


\section{Capítulo}

\section{Quase ressonância com o autovalor principal}

Neste capítulo voltamos a considerar a multiplicidade de soluções para o sistema (1.1), perto da ressonância. Em particular, mostramos que no caso em que a quase ressonância é em relação ao primeiro autovalor $\lambda_{1}$, é possível obter uma terceira solução para os problemas $(\underline{1.1} t)$. Apenas será necessário impor condições levemente mais fortes sobre a regularidade das funções $f_{1}, f_{2}, h_{1}, h_{2}$ e do conjunto $\Omega$, para garantir uma maior regularidade das soluções.

Desta forma, sejam $\Omega$ um aberto limitado de $\mathbb{R}^{N}$ de classe $\mathcal{C}^{2}$ (ver Definição 2.7), $h_{1}, h_{2} \in L^{r}(\Omega)$, para algum $r>N$ e $f_{1}, f_{2}$ funções contínuas em $\bar{\Omega} \times \mathbb{R}$, tais que existem $S>0, q \in(1,2)$, satisfazendo

$$
\left|f_{i}(x, t)\right| \leq S\left(1+|t|^{q-1}\right), \text { para cada } i=1,2 .
$$

Assumiremos ainda uma das hipóteses ( $(\mathbf{f})$ ou (F) (ver Capítulo 1). Nestas condições, os Teoremas de 3.1 a 3.4 já garantem a existência de duas soluções para os problemas $(\underline{1.1} t)$, quando a parte linear é quase ressonante. Porém, para $k=1$ (isto é, quando a parte linear é quase ressonante com o primeiro autovalor $\lambda_{1}$ ), explorando a positividade da autofunção $\phi_{1}$ e a continuidade das funções $f_{1}, f_{2}$, podemos obter uma terceira solução, distinta das outras duas.

Diremos que $\mathbf{u}=(u, v)$ é positiva (ou negativa), quando $u>0$ e $v>0$ (ou $u<0$ e 
$v<0$ ), e diremos que suas componentes possuem sinais opostos, quando $u>0$ e $v<0$ ou $u<0$ e $v>0$.

O Teorema a seguir apresenta este resultado para o caso em que $a+b$ é quase ressonante com o primeiro autovalor, enquanto $a-b$ está longe de $\sigma(-\Delta)$.

Teorema 5.1. Sejam $\Omega$ um aberto limitado de $\mathbb{R}^{N}$ de classe $\mathcal{C}^{2}, \lambda_{l}$ o primeiro autovalor acima de $a-b e$

$$
Z=\operatorname{span}\left\{\left(\phi_{1}, \phi_{1}\right)\right\}
$$

Suponhamos que $h_{1}, h_{2} \in L^{r}(\Omega)$ para algum $r>N$, e que $f_{1}, f_{2}$ sejam contínuas em $\bar{\Omega} \times \mathbb{R}$, satisfazendo as hipóteses (5.1) e (f) (ou (피)). Então

(a) dado $\delta>0$, existe $\varepsilon_{0}>0$ tal que, se $a-b \in\left(\lambda_{l-1}+\delta, \lambda_{l}-\delta\right)$ (ou $\left.a-b \in\left(-1 / \delta, \lambda_{1}-\delta\right)\right)$ e $a+b \in\left(\lambda_{1}-\varepsilon_{0}, \lambda_{1}\right)$, então o problema $(1.1+)$ possui três soluções distintas, das quais uma é positiva e outra é negativa.

(b) dado $\delta>0$, existe $\varepsilon_{1}>0$ tal que, se $a-b \in\left(\lambda_{l-1}+\delta, \lambda_{l}-\delta\right)$ (ou $\left.a-b \in\left(-1 / \delta, \lambda_{1}-\delta\right)\right)$ e $a+b \in\left(\lambda_{1}, \lambda_{1}+\varepsilon_{1}\right)$, então o problema (1.1-) possui três soluções distintas, das quais uma é positiva e outra é negativa.

Como consequência do Teorema 5.1, é possível mostrar também um resultado de existência de soluções para o caso em que $a-b$ é quase ressonante com $\lambda_{1}$, enquanto que $a+b$ está longe de $\sigma(-\Delta)$.

Teorema 5.2. Sejam $\Omega$ um aberto limitado de $\mathbb{R}^{N}$ de classe $\mathcal{C}^{2}, \lambda_{l}$ o primeiro autovalor acima de $a+b$ e

$$
Z=\operatorname{span}\left\{\left(\phi_{1},-\phi_{1}\right)\right\}
$$

Suponhamos $h_{1}, h_{2} \in L^{r}(\Omega)$ para algum $r>N, f_{1}, f_{2}$ contínuas em $\bar{\Omega} \times \mathbb{R}$, satisfazendo as hipóteses (5.1) e (f) (ou (프)). Então

(c) dado $\delta>0$, existe $\varepsilon_{0}>0$ tal que, se $a+b \in\left(\lambda_{l-1}+\delta, \lambda_{l}-\delta\right)\left(\right.$ ou $\left.a+b \in\left(-1 / \delta, \lambda_{1}-\delta\right)\right)$ e $a-b \in\left(\lambda_{1}-\varepsilon_{0}, \lambda_{1}\right)$, então o problema (1.1-) possui três soluções distintas, das quais duas delas possuem componentes com sinais opostos.

(d) dado $\delta>0$, existe $\varepsilon_{1}>0$ tal que, se $a+b \in\left(\lambda_{l-1}+\delta, \lambda_{l}-\delta\right)\left(\right.$ ou $\left.a+b \in\left(-1 / \delta, \lambda_{1}-\delta\right)\right)$ e $a-b \in\left(\lambda_{1}, \lambda_{1}+\varepsilon_{1}\right)$, então o problema $(1.1+)$ possui três soluções distintas, das quais duas delas possuem componentes com sinais opostos. 
Os resultados deste capítulo são análogos aos resultados, já citados no Capítulo (1), obtidos em [RS97, MRS97] para o problema escalar. Nesses trabalhos, os autores demonstraram a existência de três soluções distintas de (1.3), quando $\lambda$ é quase ressonante com o primeiro autovalor $\lambda_{1}, f$ e $h$ são contínuas e satisfazem condições análogas a (5.1) e (F). Em particular, eles encontraram mínimos do funcional associado a (1.3) nos cones das funções não negativas e não positivas, que correspondem a duas soluções distintas. Em seguida usaram uma geometria de passo da montanha para obter a terceira solução.

No caso do nosso sistema, devido ao funcional ser fortemente indefinido, procuraremos geometrias de sela (em vez de mínimos) do mesmo tipo das utilizadas no Capítulo 3, com apenas alguns pequenos ajustes (compare as Proposições 3.13 e 3.15 com a Proposiçã (5.3) que nos permitirão estimar a componente da solução no autoespaço quase ressonante. Feito isso, como em [RS97], poderemos obter uma condição de sinal sobre uma as duas soluções; a este ponto será fácil, por simetria, obter uma terceira solução tendo o sinal oposto.

Nas Seções 5.1 e 5.2 construímos as geometrias que nos permitirão obter as soluções, e demonstramos a existência de duas delas. Na Seção 5.3 mostramos a positividade de uma das soluções e por fim, provamos os Teoremas 5.1 e 5.2 .

\subsection{Geometrias de ponto de sela quando $a+b$ é quase ressonante inferiormente}

Dadas $f_{1}$ e $f_{2}$ contínuas em $\bar{\Omega} \times \mathbb{R}$, satisfazendo (5.1), definamos para $i=1,2$, os números $\eta_{i}=\left(-\frac{\int_{\Omega} h_{i} \phi_{1}}{\int_{\Omega} \phi_{1}}\right)^{+}$e as novas funções

$$
\tilde{f}_{i}(x, s)=\left\{\begin{array}{l}
f_{i}(x, s), \quad x \in \Omega \text { e } s \geq-1 \\
\eta_{i}, x \in \Omega \text { e } s \leq-2
\end{array}\right.
$$

de modo que sejam também contínuas em $\bar{\Omega} \times \mathbb{R}$. Notemos que desta forma, as funções $\tilde{f}_{1}, \widetilde{f}_{2}$, satisfazem (15.1), a menos de redefinir a constante $S$. Assim, denotemos

$$
\widetilde{F}_{i}(x, t)=\int_{0}^{t} \widetilde{f}_{i}(x, s) d s, \quad \text { para } i=1,2 \quad \text { e } \quad \widetilde{\mathcal{F}}(u, v)=\int_{\Omega} \widetilde{F}_{1}(x, v)+\int_{\Omega} \widetilde{F}_{2}(x, u) .
$$


Ainda, observemos que se $f_{1}, f_{2}$ satisfazem (f), então

$$
\lim _{t \rightarrow+\infty} \widetilde{f}_{i}(x, t)=+\infty, \text { uniformemente com respeito a } x \in \Omega \text { e para } i=1,2,
$$

e por outro lado, se $f_{1}, f_{2}$ satisfazem $(\underline{\mathbf{F}})$, temos

$$
\left\{\begin{array}{l}
(i) \quad \lim _{t \rightarrow+\infty} \widetilde{F}_{i}(x, t)=+\infty, \text { uniformemente com respeito a } x \in \Omega, i=1,2 \\
(\text { ii }) \quad \int_{\Omega} h_{1} \phi+h_{2} \psi=0, \quad \text { para todo }(\phi, \psi) \in Z
\end{array}\right.
$$

Consideremos o funcional $\widetilde{J}_{a, b}^{+}: E \rightarrow \mathbb{R}$, de classe $\mathcal{C}^{1}$ (ver apêndice $\bar{C}$ ), dado por

$$
\widetilde{J}_{a, b}^{+}(\mathbf{u})=\frac{1}{2} B_{a, b}(\mathbf{u}, \mathbf{u})-(\widetilde{\mathcal{F}}(\mathbf{u})+\mathcal{H}(\mathbf{u}))
$$

Fixado o autovalor $\lambda_{l}$ do enunciado do Teorema 5.1, definamos os subespaços de $E$

$$
\left\{\begin{array}{l}
V=\overline{\operatorname{span}\left\{\boldsymbol{\psi}_{i}: i \in \mathbb{Z}_{0}, \mu_{i}<0 \text { e } \mu_{i} \neq \mu_{1}\right\}} \\
Z=\overline{\operatorname{span}\left\{\boldsymbol{\psi}_{i}: i \in \mathbb{Z}_{0}, \mu_{i}=\mu_{1}\right\}} \\
W=\overline{\operatorname{span}\left\{\boldsymbol{\psi}_{i}: i \in \mathbb{Z}_{0}, \mu_{i}>0 \text { e } \mu_{i} \neq \mu_{1}\right\}}
\end{array}\right.
$$

Desta forma, vale a Proposição 5.3 abaixo, onde demonstramos que o funcional $\widetilde{J}_{a, b}^{+}$satisfaz uma geometria de sela entre os subespaços $V$ e $Z \oplus W$ (semelhante à da Proposição 3.13), enquanto o funcional $J_{a, b}^{+}$satisfaz uma geometria entre os subespaços $V \oplus Z$ e $W$, em condições de quase ressonância (semelhante à da Proposição 3.15). Ainda demonstramos as estimativas (5.9) e (5.10), que servirão para estimar os níveis críticos. Na Figura 5.1 está esboçada uma representação destas geometrias.

Proposição 5.3. Sob as hipóteses do Teorema 5.1, se $a-b \in\left(\lambda_{l-1}+\delta, \lambda_{l}-\delta\right)$ (ou $a-b \in\left(-1 / \delta, \lambda_{1}-\delta\right)$ ) e $\lambda_{1}$ é o primeiro autovalor maior que $a+b$, então existem sequências $\varepsilon_{j} \rightarrow 0^{+}$e $R_{j} \rightarrow+\infty$, ambas dependendo de $\delta$, tais que se $a+b \in\left(\lambda_{1}-\varepsilon_{j}, \lambda_{1}\right)$, então existem $E_{\delta}, D_{a+b, \delta} \in \mathbb{R}, \rho_{a+b, \delta}>R_{j}>0$, satisfazendo

$$
\begin{array}{ll}
\widetilde{J}_{a, b}^{+}(\mathbf{u}) \geq D_{a+b, \delta}, & \forall \mathbf{u} \in Z \oplus W, \\
\widetilde{J}_{a, b}^{+}(\mathbf{u})<D_{a+b, \delta}, & \forall \mathbf{u} \in \rho S_{V}, \rho \geq \rho_{a+b, \delta},
\end{array}
$$


Figura 5.1: Geometrias de ponto de sela para os funcionais $J_{a, b}^{+}$e $\widetilde{J}_{a, b}^{+}$

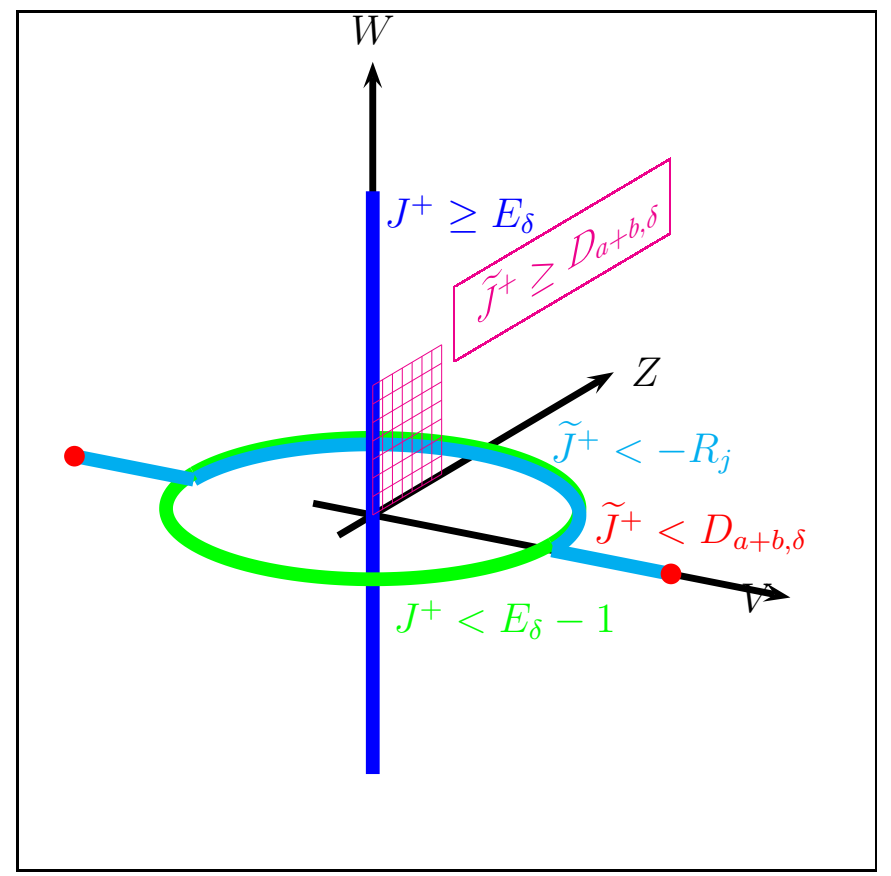

$$
\begin{aligned}
J_{a, b}^{+}(\mathbf{u}) \geq E_{\delta}, & \forall \mathbf{u} \in W, \\
J_{a, b}^{+}(\mathbf{u})<E_{\delta}-1, & \forall \mathbf{u} \in R_{j} S_{V Z}, \\
\widetilde{J}_{a, b}^{+}(\mathbf{u})<-R_{j}, & \forall \mathbf{u} \in V \operatorname{com}\|\mathbf{u}\|_{E}>R_{j}, \\
\widetilde{J}_{a, b}^{+}(\mathbf{u})<-R_{j}, & \forall \mathbf{u}=\mathbf{v}+k \boldsymbol{\psi}_{1}, \quad \text { com } \mathbf{v} \in V, k \geq 0 \text { e }\|\mathbf{u}\|_{E}=R_{j}
\end{aligned}
$$

Demonstração. Primeiramente notemos que as estimativas (5.5) e (5.6) podem ser obtidas como na prova de (3.39, 3.40), utilizando (3.28,3.29) e (3.9, 3.10), enquanto (5.7) é análoga a (3.43), e pode ser obtida pondo $\alpha=\frac{\lambda_{1}}{2}$, supondo $a+b \in\left(\lambda_{1}-\alpha, \lambda_{1}\right)$ e usando (3.31) junto com (3.9.3.10).

Além disso, a estimativa (5.8) estará satisfeita se tomarmos $\varepsilon_{j}$ suficientemente pequeno, e $R_{j}$ suficientemente grande (em particular $\varepsilon_{j}<\varepsilon_{0}$ e $R_{j}>R_{\delta}$, onde $\varepsilon_{0}, R_{\delta}$ são os mesmos dados pela Proposição 3.15).

Notemos que se $\mathbf{u} \in V$, com $\|\mathbf{u}\|_{E}>R_{j}$, por (3.47), temos

$$
\widetilde{J}_{a, b}^{+}(\mathbf{u})+R_{j} \leq \widetilde{J}_{a, b}^{+}(\mathbf{u})+\|\mathbf{u}\|_{E} \leq-G_{\alpha, \delta}\|\mathbf{u}\|_{E}^{2}+S_{0}\left(1+\|\mathbf{u}\|_{E}^{q}\right)+(H+1)\|\mathbf{u}\|_{E}
$$

Logo existe $R_{0}>0$, que pode ser escolhido dependendo apenas de $\delta$ e $\alpha$, tal que se 
$R_{j}>R_{0}$, então $\widetilde{J}_{a, b}^{+}(\mathbf{u})+R_{j}<0$, implicando (5.9) .

A fim de mostrar que existem as sequências $\left\{\varepsilon_{j}\right\}$ e $\left\{R_{j}\right\}$, satisfazendo (5.10), suponhamos por contradição que quaisquer sequências $\varepsilon_{j} \rightarrow 0^{+}$e $R_{j} \rightarrow \infty$, que satisfaçam $\varepsilon_{j}<\alpha$ e $R_{j}>R_{0}$, possuam subsequências (que denotamos da mesma forma), tais que para cada $j$, existam $a_{j}, b_{j} \in \mathbb{R}, \mathbf{v}_{j} \in V, c_{j}>0$, tais que $\mathbf{u}_{j}:=\mathbf{v}_{j}+c_{j} \boldsymbol{\psi}_{1} \in R_{j} S_{V Z}, a_{j}+b_{j} \in\left(\lambda_{1}-\varepsilon_{j}, \lambda_{1}\right)$, $a_{j}-b_{j} \in\left(\lambda_{l-1}+\delta, \lambda_{l}-\delta\right)\left(\right.$ ou $\left.a_{j}-b_{j} \in\left(-1 / \delta, \lambda_{1}-\delta\right)\right)$ e $\widetilde{J}_{a_{j}, b_{j}}^{+}\left(\mathbf{u}_{j}\right) \geq-R_{j}$.

Sem perda de generalidade, podemos supor $R_{j}^{2} \varepsilon_{j} \rightarrow 0$. Por (3.30) (e também (3.27) com $k=1$ ), temos

$$
B_{a_{j}, b_{j}}\left(\mathbf{u}_{j}, \mathbf{u}_{j}\right)<\frac{\epsilon_{j}}{\lambda_{1}} c_{j}^{2}-G_{\alpha, \delta}\left\|\mathbf{v}_{j}\right\|_{E}^{2}
$$

e assim

$$
-R_{j} \leq \widetilde{J}_{a_{j}, b_{j}}^{+}\left(\mathbf{u}_{j}\right)<\frac{\varepsilon_{j}}{\lambda_{1}} c_{j}^{2}-G_{\alpha, \delta}\left\|\mathbf{v}_{j}\right\|_{E}^{2}-\widetilde{\mathcal{F}}\left(\mathbf{u}_{j}\right)-\mathcal{H}\left(\mathbf{u}_{j}\right)
$$

Dividindo a desigualdade (5.13) por $R_{j}^{2}$ e reordenando como na prova da Proposição 3.15, concluímos

$$
\frac{\mathbf{v}_{j}}{R_{j}} \rightarrow 0, \text { em } L^{2} \times L^{2}, \text { quando } j \rightarrow \infty
$$

e

$$
\frac{c_{j}}{R_{j}} \boldsymbol{\psi}_{1} \rightarrow \boldsymbol{\psi}_{1} \text {, uniformemente, quando } j \rightarrow \infty \text {. }
$$

Escolhamos $\chi>0$ e um compacto $\omega \subset \Omega$, com $|\omega|>2 \chi$, tais que, $\phi_{1}>3 \chi$ em $\omega$. Pelo Teorema de Egorov (ver teorema (A.3) ) e (5.14), existe um subconjunto $F_{\chi}$ de $\omega$, com $\left|F_{\chi}\right|<\chi$, tal que $P_{1}\left(\frac{\mathbf{v}_{j}}{R_{j}}\right) \rightarrow 0$, uniformemente em $\omega \backslash F_{\chi}$. Desta forma usando também (5.15) , existe $j_{0}$ suficientemente grande, tal que $\frac{c_{j}}{R_{j}} \phi_{1}>\frac{1}{2} \phi_{1}$ e $\left|P_{1}\left(\frac{\mathbf{v}_{j}}{R_{j}}\right)\right|<\frac{\chi}{2}$ em $\omega \backslash F_{\chi}$, para todo $j \geq j_{0}$.

Logo, em $\omega \backslash F_{\chi}$, temos

$$
P_{1}\left(\frac{\mathbf{u}_{j}}{R_{j}}\right)=\frac{c_{j}}{R_{j}} \phi_{1}+P_{1}\left(\frac{v_{j}}{R_{j}}\right)>\frac{1}{2} \phi_{1}-\frac{\chi}{2}>\chi .
$$

Agora precisamos considerar separadamente as hipóteses $(\mathbf{f})$ e $(\mathbf{F})$.

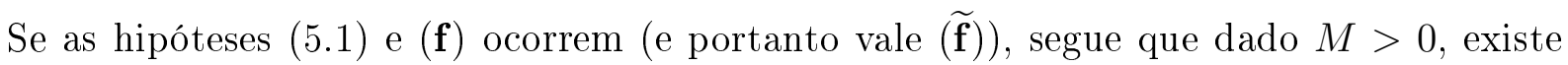
$C_{M}>0$, tal que $\widetilde{F}_{i}(x, t) \geq M t-C_{M}$, para todo $t>0$, e $\widetilde{F}_{i}(x, t) \geq-C_{M}$, para $t<0$ e 
$i=1,2$. Então, tomando $M=\frac{2+H}{\chi^{2}}$, temos para $j \geq j_{0}$, a estimativa

$$
\begin{aligned}
\int_{\Omega} \widetilde{F}_{2}\left(x, P_{1}\left(\mathbf{u}_{j}\right)\right) & =\int_{\omega \backslash F_{\chi}} \widetilde{F}_{2}\left(x, R_{j} P_{1}\left(\frac{\mathbf{u}_{j}}{R_{j}}\right)\right)+\int_{\Omega \backslash\left(\omega \backslash F_{\chi}\right)} \widetilde{F}_{2}\left(x, P_{1}\left(\mathbf{u}_{j}\right)\right) \\
& \geq M R_{j} \int_{\omega \backslash F_{\chi}} P_{1}\left(\frac{\mathbf{u}_{j}}{R_{j}}\right)-\int_{\omega \backslash F_{\chi}} C_{M}-\int_{\Omega \backslash\left(\omega \backslash F_{\chi}\right)} C_{M} \\
& \geq M R_{j} \chi^{2}-C_{M}|\Omega| .
\end{aligned}
$$

Além disso, temos

$$
\int_{\Omega} \widetilde{F}_{1}\left(x, R_{j} P_{2}\left(\frac{\mathbf{u}_{j}}{R_{j}}\right)\right) \geq-C_{M}|\Omega| .
$$

Portanto, segue destas duas estimativas, junto com (3.9), que

$$
\widetilde{\mathcal{F}}\left(\mathbf{u}_{j}\right)+\mathcal{H}\left(\mathbf{u}_{j}\right) \geq M R_{j} \chi^{2}-2 C_{M}|\Omega|-R_{j} H=2 R_{j}-2 C_{M}|\Omega| .
$$

Deste modo, voltando em (5.13), obtemos

$$
-R_{j}<\frac{\varepsilon_{j}}{\lambda_{1}} c_{j}^{2}-G_{\alpha, \delta}\left\|\mathbf{v}_{j}\right\|_{E}^{2}-2 R_{j}+2 C_{M}|\Omega|
$$

e $\log 0$

$$
R_{j}+G_{\alpha, \delta}\left\|\mathbf{v}_{j}\right\|_{E}^{2}<\frac{\varepsilon_{j}}{\lambda_{1}} c_{j}^{2}+2 C_{M}|\Omega|,
$$

o que é uma contradição, pois como $\varepsilon_{j} c_{j}^{2} \leq \varepsilon_{j} R_{j}^{2} \rightarrow 0$, quando $j \rightarrow \infty$, o lado direito da desigualdade permanece limitado, enquanto o lado esquerdo tende a $+\infty$.

Concluímos que existem sequências $\left\{\varepsilon_{j}\right\}$ e $\left\{R_{j}\right\}$, com $\varepsilon_{j} \rightarrow 0^{+}$e $R_{j} \rightarrow \infty$, satisfazendo (5.10) e (5.9) .

Agora consideramos o caso que sejam válidas as hipóteses (5.1) e (F) (e portanto vale também $(\underline{\widetilde{\mathbf{F}}})$ ).

Começamos mostrando que

$$
\lim _{R \rightarrow \infty} \inf _{j>j_{0}} \int_{\Omega} \widetilde{F}_{2}\left(x, R P_{1}\left(\widehat{\mathbf{u}}_{j}\right)\right)=+\infty
$$

Para isso, mostremos que dado $M>0$, existe $R_{0}$ suficientemente grande, tal que

$$
\int_{\Omega} \widetilde{F}_{2}\left(x, R P_{1}\left(\widehat{\mathbf{u}}_{j}\right)\right) \geq M, \quad \forall j \geq j_{0} \text { e } R \geq R_{0}
$$


Primeiramente notemos que existe $C_{F}>0$, tal que

$$
\widetilde{F}_{i}(x, t) \geq-C_{F}, \text { para todo }(x, t) \in \Omega \times \mathbb{R} \text { e } i=1,2 ;
$$

de fato, por $(\widetilde{\mathbf{F}}-i)$ e a definição de $\widetilde{f}_{i}$, podemos tomar $t_{0}>0$, tal que $\widetilde{F}_{i}(x, t) \geq 0$ para $t>t_{0}$ e $\widetilde{F}_{i}(x, t)=0$ para $t<-t_{0}$, enquanto, por (5.1),$F_{i}(x, t) \geq-S\left(t_{0}+t_{0}^{q} / q\right)$ para $|t| \leq t_{0}$.

Então, definamos $M_{1}=\frac{M+|\Omega| C_{F}}{\chi}$. Por $(\underline{\widetilde{\mathbf{F}}}-i)$, existe $s_{0}>0$ tal que,

$$
\widetilde{F}_{2}(x, s)>M_{1}, \quad \forall s>s_{0}
$$

Notemos também que para todo $R>\frac{s_{0}}{\chi}$, vale $\omega \subseteq\left\{x \in \Omega: R P_{1}\left(\widehat{\mathbf{u}}_{j}(x)\right)>s_{0}\right\}$, para $j \geq j_{0}$. Então, segue que

$$
\begin{aligned}
\int_{\Omega} \widetilde{F}_{2}\left(x, R P_{1}\left(\widehat{\mathbf{u}}_{j}\right)\right) & \geq \int_{\omega \backslash F_{\chi}} \widetilde{F}_{2}\left(x, R P_{1}\left(\widehat{\mathbf{u}}_{j}\right)\right)+\int_{\Omega \backslash\left(\omega \backslash F_{\chi}\right)}\left(-C_{F}\right) \\
& \geq \int_{\omega \backslash F_{\chi}} M_{1}-C_{F}|\Omega| \geq M_{1} \chi-C_{F}|\Omega|=M .
\end{aligned}
$$

Portanto, (5.21) está satisfeita, e uma vez que $\int_{\Omega} \widetilde{F}_{1}\left(x, R P_{2}\left(\widehat{\mathbf{u}}_{j}\right)\right)$ é limitado inferiormente, segue que

$$
\widetilde{\mathcal{F}}\left(\mathbf{u}_{j}\right)=\widetilde{\mathcal{F}}\left(R_{j} \widehat{\mathbf{u}}_{j}\right) \rightarrow+\infty, \text { quando } \quad j \rightarrow+\infty
$$

Além disso, por hipótese, temos $\mathcal{H}\left(\mathbf{u}_{j}\right)=\mathcal{H}\left(\mathbf{v}_{j}\right)$, e então

$$
G_{\alpha, \delta}\left\|\mathbf{v}_{j}\right\|_{E}^{2}+\mathcal{H}\left(\mathbf{u}_{j}\right)=G_{\alpha, \delta}\left\|\mathbf{v}_{j}\right\|_{E}^{2}+\mathcal{H}\left(\mathbf{v}_{j}\right) \geq G_{\alpha, \delta}\left\|\mathbf{v}_{j}\right\|_{E}^{2}-H\left\|\mathbf{v}_{j}\right\|_{E}
$$

Logo, existe $\delta_{2}>0$, tal que

$$
G_{\alpha, \delta}\left\|\mathbf{v}_{j}\right\|_{E}^{2}+\mathcal{H}\left(\mathbf{u}_{j}\right) \geq-\delta_{2}
$$

Por consequência, em (5.13), temos

$$
\widetilde{\mathcal{F}}\left(\mathbf{u}_{j}\right) \leq \frac{\varepsilon_{j}\left\|\mathbf{z}_{j}\right\|_{E}^{2}}{\lambda_{k}}-E_{\delta}+1+\delta_{2}
$$

o que é uma contradição, como no caso anterior, uma vez que o lado direito permanece 
limitado, enquanto o lado esquerdo tende a $+\infty$.

Concluímos que existem sequências $\left\{\varepsilon_{j}\right\}$ e $\left\{R_{j}\right\}, \operatorname{com} \varepsilon_{j} \rightarrow 0^{+}$e $R_{j} \rightarrow \infty$, satisfazendo $(5.8),(5.9)$ e $(5.10)$.

As sequências e as constantes do enunciado da proposição podem ser obtidas da seguinte forma: primeiramente tomamos $E_{\delta} \in \mathbb{R}$ satisfazendo (5.7), e então obtemos as sequências $\varepsilon_{j} \rightarrow 0^{+}$e $R_{j} \rightarrow+\infty$, satisfazendo (5.8) , (5.9) e (5.10). Notemos que, para cada $j \in \mathbb{N}$, estas estimativas são válidas para quaisquer valores de $a, b \operatorname{com} a+b \in\left(\lambda_{1}-\varepsilon_{j}, \lambda_{1}\right)$ e $a-b \in\left(\lambda_{l-1}+\delta, \lambda_{l}-\delta\right)$ (ou $a-b \in\left(-1 / \delta, \lambda_{1}-\delta\right)$ ). Desta forma, para cada $j \in \mathbb{N}$, fixando $a$ e $b$ nestas condições, podemos obter as constantes $D_{a+b, \delta} \in \mathbb{R}$ e $\rho_{a+b, \delta}>0$, tais que valem (5.5) e (5.6). Observemos que podemos tomar, para cada $j$ fixado, $\rho_{a+b, \delta}>R_{j}$.

\subsection{Existência de soluções}

Nas próximas seções consideraremos uma sequência de coeficientes $a_{j}, b_{j}$, tais que $a_{j}+b_{j} \rightarrow \lambda_{1}^{-}$e mostraremos a existência de pontos críticos dos funcionais $\widetilde{J}_{a_{j}, b_{j}}^{+}$e $J_{a_{j}, b_{j}}^{+}$, que correspondem a soluções de problemas quase ressonante com coeficientes $a_{j}, b_{j}$.

Consideremos o sistema

$$
\begin{cases}-\Delta u=a u+b v+\left(\tilde{f}_{1}(x, v)+h_{1}(x)\right) ; & \text { em } \Omega, \\ -\Delta v=b u+a v+\left(\tilde{f}_{2}(x, u)+h_{2}(x)\right) ; & \text { em } \Omega, \\ u(x)=v(x)=0 ; & \text { em } \partial \Omega .\end{cases}
$$

Indicaremos a seguir por $(\underline{1.1}+)_{j}$ e $(\underline{5.29})_{j}$ os sistemas $(\underline{1.1}+)$ e $(\underline{5.29})$ com $a=a_{j}$ e $b=b_{j}$.

Nosso objetivo é demonstrar a seguinte proposição:

Proposição 5.4. Sob as hipóteses do Teorema 5.1, dado $\delta>0$, sejam, para cada $j \in \mathbb{N}$,

- $\varepsilon_{j}>0$ os obtidos na Proposiçãa 5.3,

- $a_{j}, b_{j}$, tais que $a_{j}+b_{j} \in\left(\lambda_{1}-\varepsilon_{j}, \lambda_{1}\right)$ e $a_{j}-b_{j} \in\left(\lambda_{l-1}+\delta, \lambda_{l}-\delta\right)$ (ou $a_{j}-b_{j} \in$ $\left.\left(-1 / \delta, \lambda_{1}-\delta\right)\right)$

Então, para $j$ suficientemente grande, o problema $(1.1+)_{j}$ admite três soluções distintas, das quais uma é positiva e outra negativa. 
Vamos demonstrar primeiramente a existência de duas soluções. Para isto, procederemos como no Capítulo 3 .

De fato, uma vez que $\tilde{f}_{1}, \widetilde{f}_{2}$ satisfazem (5.1), valem os Lemas 3.17 e 3.22 também para os funcionais $\widetilde{J}_{a_{j}, b_{j}}^{+} \mid E_{n}$, para $n>2$.

Assim as desigualdades (5.5) e (5.6) definem uma geometria de sela para $\widetilde{J}_{a_{j}, b_{j}}^{+}$, entre os subespaços $V_{n}$ e $Z \oplus W_{n}$, enquanto as desigualdades (5.7) e (5.8) definem uma geometria de Sela para $J_{a_{j}, b_{j}}^{+}$, entre os subespaços $V_{n} \oplus Z$ e $W_{n}$. Obtemos então a existência dos pontos críticos $\mathbf{v}_{n}^{j}$ do funcional $J_{a_{j}, b_{j}}^{+}$restrito a $E_{n}$, e $\widetilde{\mathbf{u}}_{n}^{j}$ de $\widetilde{J}_{a_{j}, b_{j}}^{+}$restrito a $E_{n}$, nos níveis críticos $d_{n}^{j}$ e $\widetilde{c}_{n}^{j}$, respectivamente, dados por

$$
\begin{aligned}
& \widetilde{c}_{n}^{j}=\inf _{\gamma \in \Gamma_{V}^{n}} \sup _{\mathbf{u} \in \rho_{a_{j}+b_{j}, \delta} B_{V}^{n}} \widetilde{J}_{a_{j}, b_{j}}^{+}(\gamma(\mathbf{u})) \geq D_{a_{j}+b_{j}, \delta}, \\
& d_{n}^{j}=\inf _{\gamma \in \Gamma_{V Z}^{n}} \sup _{\mathbf{v} \in R_{j} B_{V Z}^{n}} J_{a_{j}, b_{j}}^{+}(\gamma(\mathbf{v})) \geq E_{\delta},
\end{aligned}
$$

onde

$$
\begin{aligned}
\Gamma_{V}^{n} & =\left\{\gamma \in \mathcal{C}\left(\rho_{a_{j}+b_{j}, \delta} B_{V}^{n}, E_{n}\right): \gamma(\mathbf{u})=\mathbf{u} \text { se }\|\mathbf{u}\|_{E}=\rho_{a_{j}+b_{j}, \delta}\right\} \\
\Gamma_{V Z}^{n} & =\left\{\gamma \in \mathcal{C}\left(R_{j} B_{V Z}^{n}, E_{n}\right): \gamma(\mathbf{v})=\mathbf{v} \text { se }\|\mathbf{v}\|_{E}=R_{j}\right\}
\end{aligned}
$$

Procedendo como no Lema 3.18, podemos mostrar que os níveis críticos $d_{n}^{j}$ são limitados superiormente por valores $T_{j}>0$, que dependem apenas de $\varepsilon_{j}$ e $\delta$, enquanto agora os níveis críticos $\widetilde{c}_{n}^{j}$ são limitados superiormente por $-R_{j}$. Para provar este último ponto, podemos usar a aplicação $\gamma_{0} \in \Gamma_{V}^{n}$, definida como

$$
\gamma_{0}(p)= \begin{cases}p ; & \text { se } R_{j} \leq\|p\|_{E} \leq \rho_{a_{j}+b_{j}, \delta} \\ p+\sqrt{\left(R_{j}\right)^{2}-\|p\|_{E}^{2}} \boldsymbol{\psi}_{1} ; & \text { se }\|p\|_{E} \leq R_{j}\end{cases}
$$

desta forma, as estimativas (5.9) e (5.10) nos fornecem a limitação superior.

Assim, para todo $j \in \mathbb{N}$, estamos em condições de passar ao limite no índice $n$, pois valem os mesmos argumentos utilizados na Seção 3.2.4. Disto segue que existem $\mathbf{v}^{j}, \widetilde{\mathbf{u}}^{j} \in$ $E$, pontos críticos dos funcionais $J_{a_{j}, b_{j}}^{+}$e $\widetilde{J}_{a_{j}, b_{j}}^{+}$, respectivamente, nos níveis $d^{j} \in\left[E_{\delta}, T_{j}\right]$ e 
$\widetilde{c}^{j} \in\left[D_{a_{j}+b_{j}, \delta},-R_{j}\right]$, tais que, a menos de subsequência,

$$
\begin{cases}\mathbf{v}_{n}^{j} \stackrel{E}{\rightarrow} \mathbf{v}^{j}, & \text { quando } n \rightarrow \infty \\ \widetilde{\mathbf{u}}_{n}^{j} \stackrel{E}{\rightarrow} \widetilde{\mathbf{u}}^{j}, & \text { quando } n \rightarrow \infty\end{cases}
$$

Desta forma, $\mathbf{v}^{j}$ é uma solução fraca de $(1.1+)_{j}$, e $\widetilde{\mathbf{u}}^{j}$ uma solução fraca de $\left([5.29){ }_{j}\right.$. Na próxima seção demonstraremos que $\widetilde{\mathbf{u}}^{j}$ deverá ser positivo para $j$ suficientemente grande: isso implicará, em vista de (5.2), que ela será também solução de $(\underline{1.1}+)_{j}$.

\subsection{Positividade da solução $\widetilde{\mathbf{u}}^{j}$}

Nesta seção consideraremos a solução fraca $\widetilde{\mathbf{u}}^{j}$ de $(\underline{5.29})_{j}$, com o objetivo de mostrar que ela é também uma solução fraca do problema $(\underline{1.1}+)_{j}$. Para isto, em virtude da definição das funções $\widetilde{f}_{1}, \widetilde{f}_{2}$, basta provarmos que $\widetilde{\mathbf{u}}^{j}$ é positiva, em $\Omega$, para $j$ suficientemente grande.

A estratégia para demonstrar que $\widetilde{\mathbf{u}}^{j}$ é positiva, é obter oportunas estimativas que mostrem que a componente de $\widetilde{\mathbf{u}}^{j}$ ortogonal ao autoespaço $Z=\operatorname{span}\left[\boldsymbol{\psi}_{1}\right]$, torna-se desprezível (na norma $\mathcal{C}^{1}$ ) em relação à componente na direção de $\boldsymbol{\psi}_{1}$.

Esta seção está dividida em duas partes: na primeira apresentamos alguns lemas contendo as estimativas necessárias para provar, na segunda parte, a positividade de $\widetilde{\mathbf{u}}^{j}$.

\subsubsection{Lemas auxiliares}

Seja $P$ a projeção ortogonal de $L^{2}$ em $\operatorname{span}\left\{\phi_{1}\right\}$ e $Q=I-P$ a projeção complementar.

Lema 5.5 (Lema 2.1 de [RS97]). Fixemos $p \geq 2$. Existem $\bar{\varepsilon}>0$ e $k_{1}>0$, ambos dependendo de $p$, tais que se $\lambda \in\left(\lambda_{1}-\bar{\varepsilon}, \lambda_{1}\right], g \in L^{p}(\Omega)$ e $u=\zeta \phi_{1}+w$, com $\zeta \in \mathbb{R} e$ $w \in Q\left(L^{2}(\Omega)\right)$, é solução do problema

$$
\left\{\begin{array}{l}
-\Delta u=\lambda u+g(x), \quad \text { em } \Omega \\
u=0, \quad \text { em } \partial \Omega
\end{array}\right.
$$

então $w \in W^{2, p}(\Omega)$ e vale a estimativa

$$
\|w\|_{W^{2, p}} \leq k_{1}\|g\|_{L^{p}}
$$


Demonstração. Pela Alternativa de Fredholm (Teorema 2.6), o problema linear

$$
\left\{\begin{array}{l}
-\Delta w=\lambda_{1} w+\widetilde{g}, \quad \text { em } \Omega \\
w=0, \quad \text { em } \partial \Omega
\end{array}\right.
$$

possui uma única solução $w \in H_{0}^{1}(\Omega) \cap Q\left(L^{2}(\Omega)\right)$, para toda $\widetilde{g} \in Q\left(L^{2}(\Omega)\right)$.

Pelo Teorema [2.8, segue que $w \in W^{2, p}(\Omega) \cap H_{0}^{1}(\Omega) \cap Q\left(L^{2}(\Omega)\right)$, desde que $\widetilde{g} \in$ $Q\left(L^{2}(\Omega)\right) \cap L^{p}(\Omega)$. Desta forma, podemos definir a aplicação

$$
T: Q\left(L^{2}(\Omega)\right) \cap L^{p}(\Omega) \rightarrow W^{2, p}(\Omega) \cap H_{0}^{1}(\Omega) \cap Q\left(L^{2}(\Omega)\right),
$$

que a cada $\widetilde{g} \in Q\left(L^{2}(\Omega)\right) \cap L^{p}(\Omega)$, associa $T(\widetilde{g})=w \in W^{2, p}(\Omega) \cap H_{0}^{1}(\Omega) \cap Q\left(L^{2}(\Omega)\right)$, satisfazendo

$$
\int_{\Omega} \nabla w \nabla \phi-\lambda_{1} \int_{\Omega} w \phi=\int_{\Omega} \widetilde{g} \phi, \quad \forall \phi \in \mathcal{C}_{c}^{\infty}(\Omega)
$$

Claramente a aplicação $T$ é linear. Mostremos que, se considerarmos a norma $\|\cdot\|_{L^{p}} \mathrm{em}$ $Q\left(L^{2}(\Omega)\right) \cap L^{p}(\Omega)$ e a norma $\|\cdot\|_{W^{2, p}}$ em $W^{2, p}(\Omega) \cap H_{0}^{1}(\Omega) \cap Q\left(L^{2}(\Omega)\right)$, então $T$ é contínua: para isto, provemos que é fechada e a continuidade será consequência do Teorema do Gráfico Fechado (Teorema A.2).

De fato, denotando por $G(T)$ o gráfico da transformação $T$, seja a sequência $\left\{\left(\widetilde{g}_{n}, w_{n}\right)\right\} \subset G(T)$, tal que $\left(\widetilde{g}_{n}, w_{n}\right) \rightarrow(\widetilde{g}, w)$, em $L^{p}(\Omega) \times W^{2, p}(\Omega) \cap H_{0}^{1}(\Omega) \cap Q\left(L^{2}(\Omega)\right)$, isto é, $T\left(\widetilde{g}_{n}\right)=w_{n}$ para todo $n \in \mathbb{N}, \widetilde{g}_{n} \rightarrow \widetilde{g}$, em $L^{p}(\Omega)$ e $w_{n} \rightarrow w$ em $W^{2, p}(\Omega) \cap H_{0}^{1}(\Omega)$. Assim, fixada uma função arbitrária $\phi \in \mathcal{C}_{c}^{\infty}(\Omega)$, como (5.38) vale para as duplas $\left(\widetilde{g}_{n}, w_{n}\right)$, tomando o limite obtemos $T(\widetilde{g})=w$ e portanto $(\widetilde{g}, w) \in G(T)$. Portanto o gráfico da transformação $T$ é fechado.

Logo $T$ é contínua e portanto existe uma constante $C_{p}>0$, dependendo de $p$, tal que

$$
\|w\|_{W^{2, p}} \leq C_{p}\|\widetilde{g}\|_{L^{p}}
$$

Assim, supondo que $u=\zeta \phi_{1}+w$ é solução fraca de (5.35), segue que $w \in Q\left(L^{2}(\Omega)\right) \cap$ $H_{0}^{1}(\Omega)$, é solução fraca de

$$
\left\{\begin{array}{l}
-\Delta w=\lambda_{1} w-\left(\lambda_{1}-\lambda\right) w+Q g, \quad \text { em } \Omega \\
w=0, \quad \text { em } \partial \Omega .
\end{array}\right.
$$


Por (5.39),

$\|w\|_{W^{2, p}} \leq C_{p}\left\|\left(\lambda_{1}-\lambda\right) w-Q g\right\|_{L^{p}} \leq C_{p}\left|\lambda_{1}-\lambda\right|\|w\|_{L^{p}}+\|Q g\|_{L^{p}} \leq C_{p} \bar{\varepsilon}\|w\|_{W^{2, p}}+C\|g\|_{L^{p}}$ $\operatorname{logo}$

$$
\left(1-C_{p} \bar{\varepsilon}\right)\|w\|_{W^{2, p}} \leq C\|g\|_{L^{p}},
$$

e o resultado segue tomando $\bar{\varepsilon}>0$, tal que $C_{p} \bar{\varepsilon}<1$.

No cálculo acima usamos a estimativa $\|Q g\|_{L^{p}} \leq C\|g\|_{L^{p}}$. De fato, escrevamos $g=$ $\alpha \phi_{1}+Q g$, com $\alpha \in \mathbb{R}$. Trivialmente (como as autofunções são todas ortogonais entre si em $\left.L^{2}(\Omega)\right)$, temos $\|Q g\|_{L^{2}} \leq\|g\|_{L^{2}}$, de onde segue que $|\alpha|=\|g-Q g\|_{L^{2}} \leq 2\|g\|_{L^{2}}$. Pela imersão contínua $L^{p}(\Omega) \hookrightarrow L^{2}(\Omega)$, temos $|\alpha| \leq 2 C\|g\|_{L^{p}}$. Portanto

$$
\|Q g\|_{L^{p}} \leq\|g\|_{L^{p}}+|\alpha|\|\phi\|_{L^{p}} \leq\left(1+2 C\|\phi\|_{L^{p}}\right)\|g\|_{L^{p}} .
$$

Lema 5.6. Fixemos $p \geq 2, \delta>0$ e $\lambda_{l-1}, \lambda_{l}$ autovalores distintos de $(-\Delta)$. Então existe $k_{2}>0$ dependendo de $p, l$ e de $\delta$, tal que se $\mu \in\left(\lambda_{l-1}+\delta, \lambda_{l}-\delta\right)$ (ou $\left.\mu \in\left(-1 / \delta, \lambda_{1}-\delta\right)\right)$, vale

$$
\|u\|_{W^{2, p}} \leq k_{2}\|\Delta u+\mu u\|_{L^{p}},
$$

para todo $u \in W^{2, p}(\Omega) \cap H_{0}^{1}(\Omega)$.

Demonstração. A prova é feita para o caso $\mu \in\left(\lambda_{l-1}+\delta, \lambda_{l}-\delta\right)$, uma vez que o caso $\mu \in\left(-1 / \delta, \lambda_{1}-\delta\right)$ segue de modo análogo. Suponhamos por contradição que existam sequências

$$
\left\{\mu_{n}\right\} \subset\left(\lambda_{l-1}+\delta, \lambda_{l}-\delta\right) \quad \text { e } \quad\left\{u_{n}\right\} \subset W^{2, p}(\Omega) \cap H_{0}^{1}(\Omega)
$$

tais que $\left\|u_{n}\right\|_{W^{2, p}}=1$ e $\left\|\Delta u_{n}+\mu_{n} u_{n}\right\|_{L^{p}(\Omega)} \rightarrow 0$, quando $n \rightarrow \infty$.

Assim, passando a subsequência, temos

$$
\left\{\begin{array}{l}
\mu_{n} \rightarrow \mu \in\left[\lambda_{l-1}+\delta, \lambda_{l}-\delta\right], \\
u_{n} \rightarrow u \in W^{2, p}(\Omega), \\
u_{n} \rightarrow u \in L^{p}(\Omega) .
\end{array}\right.
$$


Seja uma função arbitrária $\phi \in \mathcal{C}_{c}^{\infty}(\Omega)$, em particular, temos $\phi \in L^{p /(p-1)}(\Omega)$, e então

$$
\int_{\Omega}\left(\Delta u_{n}+\mu_{n} u_{n}\right) \phi=\int_{\Omega} \Delta u_{n} \phi+\mu_{n} \int_{\Omega} u_{n} \phi \rightarrow \int_{\Omega} \Delta u \phi+\mu \int_{\Omega} u \phi=\int_{\Omega}(\Delta u+\mu u) \phi
$$

Por outro lado, pela Desigualdade de Hölder, temos

$$
\left|\int_{\Omega}\left(\Delta u_{n}+\mu_{n} u_{n}\right) \phi\right| \leq \int_{\Omega}\left|\left(\Delta u_{n}+\mu_{n} u_{n}\right) \phi\right| \leq\left\|\Delta u_{n}+\mu_{n} u_{n}\right\|_{L^{p}}\|\phi\|_{L^{p /(p-1)}} \rightarrow 0, \quad n \rightarrow \infty .
$$

Segue assim que $\int_{\Omega}(\Delta u+\mu u) \phi=0$, para toda $\phi \in \mathcal{C}_{c}^{\infty}(\Omega)$. Logo (lembremos que $u \in$ $H_{0}^{1}(\Omega)$ e portanto se anula na fronteira) $u$ é solução de

$$
\begin{cases}-\Delta u=\mu u, & \text { em } \quad \Omega, \\ u=0, & \text { em } \quad \partial \Omega,\end{cases}
$$

o que implica que $u=0$, uma vez que $\mu \notin \sigma(-\Delta)$.

Por outro lado, como $u_{n} \in W^{2, p}, f(x)=-\Delta u_{n}(x)-\mu_{n} u_{n}(x) \in L^{p}$, aplicando o Teorema 2.8 obtemos a estimativa a priori,

$$
\left\|u_{n}\right\|_{W^{2, p}} \leq C\left(\left\|u_{n}\right\|_{L^{p}}+\left\|\Delta u_{n}+\mu_{n} u_{n}\right\|_{L^{p}}\right), \quad \text { para todo } n \in \mathbb{N},
$$

onde a constante $C>0$ não depende do índice $n$ (depende de $p, \Omega$ e de $l$ ). Como o lado esquerdo é igual a 1 , enquanto o lado direito tende a $C\|u\|_{L^{p}}$, quando $n \rightarrow \infty$, segue que $\|u\|_{L^{p}} \geq \frac{1}{C}>0$, o que é uma contradição com $u=0$.

Graças ao Lema 5.6, podemos obter um resultado análogo ao Lema 5.5 para o caso de um sistema linear perto da ressonância.

Lema 5.7. Fixemos $p \geq 2, \delta>0$ e $\lambda_{l-1}, \lambda_{l}$ autovalores distintos de $(-\Delta)$. Então existem $\bar{\varepsilon}>0$ e $k_{0}>0$, dependendo de $l, \delta$ e de $p$, tais que, se $a-b \in\left(\lambda_{l-1}+\delta, \lambda_{l}-\delta\right)$ (ou $\left.a-b \in\left(-1 / \delta, \lambda_{1}-\delta\right)\right), a+b \in\left(\lambda_{1}-\bar{\varepsilon}, \lambda_{1}\right), \mathbf{g}=\left(g_{1}, g_{2}\right) \in L^{p} \times L^{p} e \mathbf{u}=(u, v)=\beta \boldsymbol{\psi}_{1}+\boldsymbol{\omega}$, com $\beta \in \mathbb{R}$ e $\boldsymbol{\omega} \in V \oplus W$, é solução do problema

$$
\left\{\begin{array}{l}
-\Delta u=a u+b v+g_{1}(x), \quad \text { em } \quad \Omega, \\
-\Delta v=b u+a v+g_{2}(x), \quad \text { em } \quad \Omega \\
u=v=0, \quad \text { em } \quad \partial \Omega,
\end{array}\right.
$$


então vale a estimativa,

$$
\|\boldsymbol{\omega}\|_{W^{2, p} \times W^{2, p}} \leq k_{0}\|\mathbf{g}\|_{L^{p} \times L^{p}} .
$$

Demonstração. Somando e subtraindo as equações de (5.48), temos

$$
\left\{\begin{array}{l}
-\nabla s=(a+b) s+g_{1}(x)+g_{2}(x), \quad \text { em } \quad \Omega \\
-\nabla d=(a-b) d+g_{1}(x)-g_{2}(x), \quad \text { em } \quad \Omega \\
s=d=0, \quad \text { em } \quad \partial \Omega
\end{array}\right.
$$

onde $s=u+v$ e $d=u-v$. Assim, para a primeira equação, segue do Lema 5.5 que existem $\bar{\varepsilon}>0$ e $k_{1}>0$ (dependendo de $p$ ), tais que se $a+b \in\left(\lambda_{1}-\bar{\varepsilon}, \lambda_{1}\right.$ ], vale a estimativa

$$
\|Q(s)\|_{W^{2, p}} \leq k_{1}\left\|g_{1}+g_{2}\right\|_{L^{p}} .
$$

Para a segunda equação, segue do Lema 5.6, que existe uma constante $k_{2}>0$ (dependendo de $p, l$ e $\delta)$, tal que

$$
\|d\|_{W^{2, p}} \leq k_{2}\left\|g_{1}-g_{2}\right\|_{L^{p}} .
$$

Escrevendo $\boldsymbol{\omega}=\left(\omega_{1}, \omega_{2}\right)$, temos $Q(s)=\omega_{1}+\omega_{2}$ e $d=\omega_{1}-\omega_{2}$. Assim $\boldsymbol{\omega}=$ $\left(\frac{Q(s)+d}{2}, \frac{Q(s)-d}{2}\right)$, e pela primeira desigualdade do Lema A.9 e a desigualdade triangular, temos

$$
\begin{aligned}
\|\boldsymbol{\omega}\|_{W^{2, p} \times W^{2, p}} & =\left(\left\|\frac{Q(s)+d}{2}\right\|_{W^{2, p}}^{p}+\left\|\frac{Q(s)-d}{2}\right\|_{W^{2, p}}^{p}\right)^{1 / p} \\
& \leq\left\|\frac{Q(s)+d}{2}\right\|_{W^{2, p}}+\left\|\frac{Q(s)-d}{2}\right\|_{W^{2, p}} \leq\left(\|Q(s)\|_{W^{2, p}}+\|d\|_{W^{2, p}}\right) \\
& \leq\left(k_{1}\left\|g_{1}+g_{2}\right\|_{L^{p}}+k_{2}\left\|g_{1}-g_{2}\right\|_{L^{p}}\right) \leq\left(k_{1}+k_{2}\right)\left(\left\|g_{1}\right\|_{L^{p}}+\left\|g_{2}\right\|_{L^{p}}\right),
\end{aligned}
$$

enfim, pela segunda desigualdade do Lema \$.9, concluímos que

$$
\|\boldsymbol{\omega}\|_{W^{2, p} \times W^{2, p}} \leq\left(k_{1}+k_{2}\right) 2^{\frac{p-1}{p}}\|\mathbf{g}\|_{L^{p} \times L^{p}} .
$$

Tomando $k_{0}=2^{\frac{p-1}{p}}\left(k_{1}+k_{2}\right)$ concluímos a demonstração.

Usaremos o Lema 5.7 para demonstrar o lema a seguir, onde obtemos uma estimativa na norma $\mathcal{C}^{1}$ da componente ortogonal a $Z$ de uma solução fraca de (․․29), em função da componente em $Z$. 
Lema 5.8. Sejam $f_{1}, f_{2}$ contínuas em $\bar{\Omega} \times \mathbb{R}$ satisfazendo (5.1), $h_{1}, h_{2} \in L^{r}(\Omega)$, para algum $r>N$, e fixemos $\delta>0$ e $\lambda_{l-1}, \lambda_{l}$ autovalores distintos de $(-\Delta)$. Seja $\bar{\varepsilon}$ o obtido no Lema 5.7, com $p=r$. Dado $\eta>0$, existe $\widetilde{C}_{\eta}>0$, dependendo de $\eta, l, \delta$ e de r, tal que se $a-b \in\left(\lambda_{l-1}+\delta, \lambda_{l}-\delta\right)\left(\right.$ ou $\left.a-b \in\left(-1 / \delta, \lambda_{1}-\delta\right)\right), a+b \in\left(\lambda_{1}-\bar{\varepsilon}, \lambda_{1}\right)$ e $\widetilde{\mathbf{u}}=\beta \boldsymbol{\psi}_{1}+\boldsymbol{\omega}$, com $\beta \in \mathbb{R} e \boldsymbol{\omega} \in V \oplus W$, é um ponto crítico do funcional $\widetilde{J}_{a, b}^{+}$, então vale a estimativa

$$
\|\boldsymbol{\omega}\|_{\mathcal{C}^{1} \times \mathcal{C}^{1}} \leq \eta|\beta|+\widetilde{C}_{\eta}
$$

Demonstração. Seja $\widetilde{\mathbf{u}}=(\widetilde{u}, \widetilde{v})$ um ponto crítico do funcional $\widetilde{J}_{a, b}^{+}$, e então uma solução fraca do problema (5.29). Denotemos

$$
\widetilde{\mathbf{f}}(\widetilde{\mathbf{u}})=\left(\widetilde{f}_{1}(x, \widetilde{v}(x)), \widetilde{f}_{2}(x, \widetilde{u}(x))\right) \quad \text { e } \quad \mathbf{h}=\left(h_{1}(x), h_{2}(x)\right) .
$$

Notemos que pelas hipóteses sobre as funções $f_{1}, f_{2}, h_{1}, h_{2}$, se $\widetilde{u}, \widetilde{v} \in L^{p}(\Omega)$ para algum $p \in[1, r]$, temos $\widetilde{\mathbf{f}}(\widetilde{\mathbf{u}}), \mathbf{h} \in L^{p}(\Omega) \times L^{p}(\Omega)$, e assim pelo Teorema 2.8 concluímos que $\widetilde{u}, \widetilde{v} \in W^{2, p}(\Omega)$.

Afirmamos que $\widetilde{\mathbf{u}} \in W^{2, r}(\Omega) \times W^{2, r}(\Omega)$. A ideia para demonstrar esta afirmação é combinar as imersões de Sobolev (ver apêndice A.4) com o Teorema 2.8, e iterar o quanto for necessário, até obtermos $\widetilde{u}, \widetilde{v} \in W^{2, p}(\Omega)$ para algum $p \geq N / 2$. Assim $N-2 p \leq 0$ de modo que valerá a imersão $W^{2, p}(\Omega) \hookrightarrow L^{r}(\Omega)$, e portanto do Teorema 2.8 concluiremos que $\widetilde{u}, \widetilde{v} \in W^{2, r}(\Omega)$. Para começar as iterações, observamos que por ser solução fraca, temos $\widetilde{u}, \widetilde{v} \in W^{2,2}(\Omega)$ (basta aplicar o Teorema 2.8, com $p=2$ ).

- Caso $N \leq 4$, o resultado já está provado;

- caso $N>4$, pela imersão $W^{2,2}(\Omega) \hookrightarrow L^{p_{1}}(\Omega)$, onde $p_{1}=\frac{2 N}{N-4}>2$, temos $\widetilde{u}, \widetilde{v} \in$ $L^{p_{1}}(\Omega)$ e portanto $\widetilde{u}, \widetilde{v} \in W^{2, p_{1}}(\Omega)$. Se $N-2 p_{1}>0$, então utilizamos a imersão $W^{2, p_{1}}(\Omega) \hookrightarrow L^{p_{2}}(\Omega)$, onde $p_{2}=\frac{N p_{1}}{N-2 p_{1}}$, e portanto $\widetilde{u}, \widetilde{v} \in W^{2, p_{2}}(\Omega)$. Repetindo este raciocínio, obtemos que $\widetilde{u}, \widetilde{v} \in W^{2, p_{i}}(\Omega)$, onde a sequência $\left\{p_{i}\right\}$ é definida recursivamente por $p_{0}:=2$ e $p_{i+1}=\frac{N p_{i}}{N-2 p_{i}}$. Para concluir a prova, basta mostrarmos que existe $i_{0} \in \mathbb{N}$, tal que $p_{i_{0}} \geq N / 2$. Para isto, suponhamos por contradição que $p_{i} \in(2, N / 2)$ para todo $i \in \mathbb{N}$, o que implica que $p_{i+1}>p_{i}$. Assim a sequência $\left\{p_{i}\right\}$ seria crescente e limitada, e portanto convergiria para $L \in[2, N / 2]$. Porém, tomando limite na fórmula de recursão, temos $L=\lim \frac{N p_{i}}{N-2 p_{i}}$ que implica $L=0$, 
uma contradição.

Portanto está provado que $\widetilde{\mathbf{u}} \in W^{2, r}(\Omega) \times W^{2, r}(\Omega)$.

Uma vez que $r>N$, vale a imersão contínua $W^{2, r}(\Omega) \hookrightarrow \mathcal{C}^{1}(\bar{\Omega})$ (ver apêndice A.4), e pelo Lema 5.7, existe $k_{0}>0$, tal que,

$$
\|\boldsymbol{\omega}\|_{W^{2, r} \times W^{2, r}} \leq k_{0}\|\widetilde{\mathbf{f}}(\widetilde{\mathbf{u}})+\mathbf{h}\|_{L^{r} \times L^{r}}
$$

Portanto segue

$$
\|\boldsymbol{\omega}\|_{\mathcal{C}^{1} \times \mathcal{C}^{1}} \leq C\|\boldsymbol{\omega}\|_{W^{2, r} \times W^{2, r}} \leq C k_{0}\|\widetilde{\mathbf{f}}(\widetilde{\mathbf{u}})+\mathbf{h}\|_{L^{r} \times L^{r}} \leq C k_{0}\|\widetilde{\mathbf{f}}(\widetilde{\mathbf{u}})\|_{L^{r} \times L^{r}}+C k_{0} H_{r}
$$

onde $H_{r}=\|\mathbf{h}\|_{L^{r} \times L^{r}}$.

Agora notemos que por (5.1), dado $\gamma>0$, existe $D_{\gamma}>0$, tal que $\left|\tilde{f}_{i}(x, t)\right| \leq \gamma|t|+D_{\gamma}$, para todo $t \in \mathbb{R}$ e $i=1,2$. Logo

$$
\|\widetilde{\mathbf{f}}(\widetilde{\mathbf{u}})\|_{L^{r} \times L^{r}} \leq \gamma\|\widetilde{\mathbf{u}}\|_{L^{r} \times L^{r}}+C_{1} D_{\gamma}
$$

Assim, combinando esta desigualdade com (5.56), segue

$$
\begin{aligned}
\|\boldsymbol{\omega}\|_{\mathcal{C}^{1} \times \mathcal{C}^{1}} & \leq C k_{0} \gamma\|\widetilde{\mathbf{u}}\|_{L^{r} \times L^{r}}+C k_{0}\left(H_{r}+C_{1} D_{\gamma}\right) \\
& \leq C k_{0} \gamma|\beta|\left\|\boldsymbol{\psi}_{1}\right\|_{L^{r} \times L^{r}}+C k_{0} \gamma\|\boldsymbol{\omega}\|_{L^{r} \times L^{r}}+\widetilde{D}_{\gamma} \\
& \leq C k_{0} \gamma\left\|\boldsymbol{\psi}_{1}\right\|_{L^{r} \times L^{r}}|\beta|+C_{2} k_{0} \gamma\|\boldsymbol{\omega}\|_{\mathcal{C}^{1} \times \mathcal{C}^{1}}+\widetilde{D}_{\gamma}
\end{aligned}
$$

onde $\widetilde{D}_{\gamma}=C k_{0}\left(H_{r}+C_{1} D_{\gamma}\right)$.

Logo, dado $\eta>0$, podemos tomar $\gamma>0$, tal que $C k_{0} \gamma\left\|\boldsymbol{\psi}_{1}\right\|_{L^{r} \times L^{r}}<\frac{\eta}{2}$ e $C_{2} k_{0} \gamma<\frac{1}{2}$, dessa maneira podemos levar para esquerda o segundo termo da direita e obter o resultado desejado com $\widetilde{C}_{\eta}=2 \widetilde{D}_{\gamma}$.

Notemos ainda que tal escolha para $\gamma\left(\right.$ e $\left.\log 0 \widetilde{C}_{\eta}\right)$ depende de $k_{0}$ e $r$, logo de $\delta, l, r$.

\subsubsection{Mostrando solução positiva}

Nesta seção mostramos que existe $j_{0}$ suficientemente grande, tal que $\widetilde{\mathbf{u}}^{j}$ é positiva em $\Omega$, para todo $j \geq j_{0}$. Lembremos (ver Seção 5.2 ) que $\widetilde{\mathbf{u}}^{j}$ é ponto crítico do funcional $\widetilde{J}_{a_{j}, b_{j}}^{+}$ 
no nível crítico $\widetilde{c}^{j} \leq-R_{j}$, e uma vez que $R_{j} \rightarrow+\infty$, temos

$$
\widetilde{J}_{a_{j}, b_{j}}^{+}\left(\widetilde{\mathbf{u}}^{j}\right)=\widetilde{c}^{j} \rightarrow-\infty, \quad \text { quando } j \rightarrow \infty
$$

Para cada $j \in \mathbb{N}$, denotemos

$$
\widetilde{\mathbf{u}}^{j}=\beta_{j} \boldsymbol{\psi}_{1}+\boldsymbol{\omega}_{j}
$$

onde $\beta_{j} \in \mathbb{R}$ e $\boldsymbol{\omega}_{j} \in V \oplus W$. Notemos que $\left|\beta_{j}\right| \rightarrow+\infty$, quando $j \rightarrow \infty$, pois se a sequência $\left\{\beta_{j}\right\}$ fosse limitada, então pelo Lema 5.8, a sequência $\left\{\boldsymbol{\omega}_{j}\right\}$ seria limitada em $E$ e consequentemente, por (5.58), $\left\{\widetilde{\mathbf{u}}^{j}\right\}$ também seria limitada em $E$. Assim deveríamos ter $\widetilde{J}_{a_{j}, b_{j}}^{+}\left(\widetilde{\mathbf{u}}^{j}\right)$ limitado, uma vez que os coeficientes $a_{j}, b_{j}$ variam em um compacto fixado, contradizendo (5.57).

Desta forma, pelo Lema 5.8, temos

$$
\limsup _{j \rightarrow \infty} \frac{\left\|\boldsymbol{\omega}_{j}\right\|_{\mathcal{C}^{1} \times \mathcal{C}^{1}}}{\left|\beta_{j}\right|} \leq \limsup _{j \rightarrow \infty}\left(\eta+\frac{\widetilde{C}_{\eta}}{\left|\beta_{j}\right|}\right)=\eta
$$

Pela arbitrariedade de $\eta$, segue

$$
\frac{\boldsymbol{\omega}_{j}}{\beta_{j}} \rightarrow 0, \text { em } \mathcal{C}^{1}(\bar{\Omega}) \times \mathcal{C}^{1}(\bar{\Omega}), \text { quando } j \rightarrow \infty
$$

e portanto

$$
\frac{\widetilde{\mathbf{u}}^{j}}{\beta_{j}}=\boldsymbol{\psi}_{1}+\frac{\boldsymbol{\omega}_{j}}{\beta_{j}} \rightarrow \boldsymbol{\psi}_{1} \text {, em } \mathcal{C}^{1}(\bar{\Omega}) \times \mathcal{C}^{1}(\bar{\Omega}) \text {, quando } j \rightarrow \infty
$$

Uma vez que $\boldsymbol{\psi}_{1}$ é positiva com derivada destacada de zero no bordo, existe $j_{0}$, tal que para todo $j \geq j_{0}$, $\widetilde{\mathbf{u}}^{j}$ é positiva, quando $\beta_{j}>0$, ou negativa, quando $\beta_{j}<0$.

A fim de concluir que a solução $\widetilde{\mathbf{u}}^{j}$ é positiva, devemos mostrar o seguinte lema.

Lema 5.9. Para cada $j \in \mathbb{N}$, seja $\beta_{j}$ dado por (5.58). Então $\beta_{j} \rightarrow+\infty$, quando $j \rightarrow \infty$.

Demonstração. Já sabemos que a sequência $\left|\beta_{j}\right| \rightarrow+\infty$. Então devemos apenas mostrar que nenhuma subsequência pode tender a $-\infty$. Notemos primeiramente que pela definição de $\widetilde{f}_{1}, \widetilde{f}_{2}$ e por serem contínuas, existe $M>0$ tal que, para $i=1,2$, vale

$$
\left|\tilde{f}_{i}(x, t)\right| \leq M, \quad \text { para todo } x \in \Omega, t \leq 0
$$


Pela definição de $\widetilde{F}_{i}(x, t)$, vale também

$$
\begin{array}{r}
\left|\widetilde{F}_{i}(x, t)\right| \leq M|t| \leq 2 M, \quad \text { para todo } x \in \Omega, t \in[-2,0], \\
\widetilde{F}_{i}(x, t) \leq 2 M+\eta_{i}(t+2) \leq 2 M, \quad \text { para todo } x \in \Omega, t \leq-2 .
\end{array}
$$

Suponhamos que $\beta_{j} \rightarrow-\infty$, quando $j \rightarrow \infty$.

Neste caso, por (5.61), $\widetilde{\mathbf{u}}^{j}$ é negativa, para $j$ suficientemente grande. Assim, por (5.62), temos

$$
\left\|\widetilde{\mathbf{f}}\left(\widetilde{\mathbf{u}}^{j}\right)\right\|_{L^{p} \times L^{p}} \leq 2 M|\Omega|^{\frac{1}{p}}
$$

Portanto, pelo Lema $5.7(\operatorname{com} p=2)$, segue

$$
\left\|\boldsymbol{\omega}_{j}\right\|_{E} \leq\left\|\boldsymbol{\omega}_{j}\right\|_{W^{2,2} \times W^{2,2}} \leq k_{0}\left\|\widetilde{\mathbf{f}}\left(\widetilde{\mathbf{u}}^{j}\right)+\mathbf{h}\right\|_{\left[L^{2}\right]^{2}} \leq 2 M k_{0}|\Omega|^{\frac{1}{2}}+k_{0}\|\mathbf{h}\|_{\left[L^{2}\right]^{2}}<\infty
$$

Uma vez que

$$
B_{a_{j}, b_{j}}\left(\widetilde{\mathbf{u}}^{j}, \widetilde{\mathbf{u}}^{j}\right)=B_{a_{j}, b_{j}}\left(\beta_{j} \boldsymbol{\psi}_{1}, \beta_{j} \boldsymbol{\psi}_{1}\right)+B_{a_{j}, b_{j}}\left(\boldsymbol{\omega}_{j}, \boldsymbol{\omega}_{j}\right)
$$

temos

$$
\widetilde{J}_{a_{j}, b_{j}}^{+}\left(\widetilde{\mathbf{u}}^{j}\right)=\frac{\beta_{j}^{2}}{2} B_{a_{j}, b_{j}}\left(\boldsymbol{\psi}_{1}, \boldsymbol{\psi}_{1}\right)+\frac{1}{2} B_{a_{j}, b_{j}}\left(\boldsymbol{\omega}_{j}, \boldsymbol{\omega}_{j}\right)-\left(\widetilde{\mathcal{F}}\left(\widetilde{\mathbf{u}}^{j}\right)+\mathcal{H}\left(\widetilde{\mathbf{u}}^{j}\right)\right) .
$$

Precisamos de uma oportuna estimativa para o termo $\widetilde{\mathcal{F}}\left(\widetilde{\mathbf{u}}^{j}\right)+\mathcal{H}\left(\widetilde{\mathbf{u}}^{j}\right)$. Para isto, denote$\operatorname{mos} \boldsymbol{\omega}_{j}=\left(P_{1} \boldsymbol{\omega}_{j}, P_{2} \boldsymbol{\omega}_{j}\right)$ e $\widetilde{\mathbf{u}}^{j}=\left(\widetilde{u}_{j}, \widetilde{v}_{j}\right)=\left(\beta_{j} \phi_{1}+P_{1} \boldsymbol{\omega}_{j}, \beta_{j} \phi_{1}+P_{2} \boldsymbol{\omega}_{j}\right)$, e observemos que, por $(\underline{5.63})$ e (5.64), vale

$$
\begin{aligned}
\int_{\Omega} \widetilde{F}_{1}\left(x, \widetilde{v}_{j}\right) & =\int_{\left\{\widetilde{v}_{j} \leq-2\right\}} \widetilde{F}_{1}\left(x, \widetilde{v}_{j}\right)+\int_{\left\{-2<\widetilde{v}_{j} \leq 0\right\}} \widetilde{F}_{1}\left(x, \widetilde{v}_{j}\right) \\
& \leq \int_{\left\{\widetilde{v}_{j} \leq-2\right\}}\left(2 M+\eta_{1}\left(\widetilde{v}_{j}+2\right)\right)+\int_{\left\{-2<\widetilde{v}_{j} \leq 0\right\}} 2 M \\
& \leq \int_{\Omega} 2 M+\int_{\left\{\widetilde{v}_{j} \leq-2\right\}} \eta_{1}\left(\widetilde{v}_{j}+2\right),
\end{aligned}
$$


e uma vez que $\int_{\left\{\widetilde{v}_{j} \leq-2\right\}}\left(\widetilde{v}_{j}+2\right) \leq \int_{\Omega}\left(\widetilde{v}_{j}+2\right)=\int_{\Omega} \widetilde{v}_{j}+2|\Omega|$ e $\eta_{1} \geq 0$, temos

$$
\int_{\Omega} \widetilde{F}_{1}\left(x, \widetilde{v}_{j}\right) \leq 2\left(M+\eta_{1}\right)|\Omega|+\eta_{1} \int_{\Omega} \widetilde{v}_{j}
$$

Da mesma forma,

$$
\int_{\Omega} \widetilde{F}_{2}\left(x, \widetilde{u}_{j}\right) \leq 2\left(M+\eta_{2}\right)|\Omega|+\eta_{2} \int_{\Omega} \widetilde{u}_{j}
$$

e assim, por (5.72,5.73), temos

$$
\widetilde{\mathcal{F}}\left(\widetilde{\mathbf{u}}^{j}\right) \leq 2\left(2 M+\eta_{1}+\eta_{2}\right)|\Omega|+\eta_{1} \int_{\Omega} \widetilde{v}_{j}+\eta_{2} \int_{\Omega} \widetilde{u}_{j}
$$

Portanto, uma vez que $\mathcal{H}\left(\widetilde{\mathbf{u}}^{j}\right)=\beta_{j} \mathcal{H}\left(\boldsymbol{\psi}_{1}\right)+\mathcal{H}\left(\boldsymbol{\omega}_{j}\right)$, segue por (5.74) e (3.9),

$$
\begin{aligned}
\widetilde{\mathcal{F}}\left(\widetilde{\mathbf{u}}^{j}\right)+\widetilde{\mathcal{H}}\left(\widetilde{\mathbf{u}}^{j}\right) \leq & \eta_{1} \int_{\Omega} \widetilde{v}_{j}+\eta_{2} \int_{\Omega} \widetilde{u}_{j}+2\left(2 M+\eta_{1}+\eta_{2}\right)|\Omega|+\beta_{j} \mathcal{H}\left(\boldsymbol{\psi}_{1}\right)+\mathcal{H}\left(\boldsymbol{\omega}_{j}\right) \\
= & \beta_{j}\left(\eta_{1} \int_{\Omega} \phi_{1}+\eta_{2} \int_{\Omega} \phi_{1}+\int_{\Omega} h_{1} \phi_{1}+\int_{\Omega} h_{2} \phi_{1}\right) \\
& +\eta_{1} \int_{\Omega} P_{2} \boldsymbol{\omega}_{j}+\eta_{2} \int_{\Omega} P_{1} \boldsymbol{\omega}_{j}+\mathcal{H}\left(\boldsymbol{\omega}_{j}\right)+2\left(2 M+\eta_{1}+\eta_{2}\right)|\Omega| \\
\leq & \left(\eta_{1}+\eta_{2}+H\right)\left\|\boldsymbol{\omega}_{j}\right\|_{E}+2\left(2 M+\eta_{1}+\eta_{2}\right)|\Omega|
\end{aligned}
$$

de fato, $\beta_{j}\left(\eta_{1} \int_{\Omega} \phi_{1}+\int_{\Omega} h_{1} \phi_{1}\right)+\beta_{j}\left(\eta_{2} \int_{\Omega} \phi_{1}+\int_{\Omega} h_{2} \phi_{1}\right) \leq 0$, por como foram definidos $\eta_{1}, \eta_{2}$ e sendo que $\beta_{j} \leq 0$.

Desta forma, voltando em (5.68), pela estimativa (5.75), segue

$$
\widetilde{J}_{a_{j}, b_{j}}^{+}\left(\widetilde{\mathbf{u}}^{j}\right) \geq \frac{\beta_{j}^{2}}{2} B_{a_{j}, b_{j}}\left(\boldsymbol{\psi}_{1}, \boldsymbol{\psi}_{1}\right)+\frac{1}{2} B_{a_{j}, b_{j}}\left(\boldsymbol{\omega}_{j}, \boldsymbol{\omega}_{j}\right)-\left(\eta_{1}+\eta_{2}+H\right)\left\|\boldsymbol{\omega}_{j}\right\|_{E}-2\left(2 M+\eta_{1}+\eta_{2}\right)|\Omega|
$$

Uma vez que $B_{a_{j}, b_{j}}\left(\boldsymbol{\psi}_{1}, \boldsymbol{\psi}_{1}\right)=\frac{\lambda_{1}-\left(a_{j}+b_{j}\right)}{\lambda_{1}}>0$, e o termo $\boldsymbol{\omega}_{j}$ é limitado em $E$ por (5.66) ), chegamos a uma contradição com (5.57).

Como antecipamos, a consequência deste lema é que $\widetilde{\mathbf{u}}^{j}$ é positiva, para todo $j \geq j_{0}$. Pela definição de $\widetilde{f}_{1}, \widetilde{f}_{2}$, e lembrando que $\widetilde{\mathbf{u}}^{j}$ é solução fraca de $(\underline{5.29})_{j}$, segue que é também uma solução fraca de $(1.1+)_{j}$ e que

$$
J_{a_{j}, b_{j}}^{+}\left(\widetilde{\mathbf{u}}^{j}\right)=\widetilde{J}_{a_{j}, b_{j}}^{+}\left(\widetilde{\mathbf{u}}^{j}\right) \in\left[D_{a_{j}+b_{j}, \delta},-R_{j}\right] .
$$


Estamos agora em condições de demonstrar a Proposição 5.4.

Demonstração da Proposição 5.4. As funções $\mathbf{v}^{j}$ e $\widetilde{\mathbf{u}}^{j}$ são soluções de $(1.1+)_{j}$, sendo ainda a segunda delas positiva. Como $J_{a_{j}, b_{j}}^{+}\left(\mathbf{v}^{j}\right) \geq E_{\delta}$ enquanto $J_{a_{j}, b_{j}}^{+}\left(\widetilde{\mathbf{u}}^{j}\right) \rightarrow-\infty$, elas serão distintas, para $j$ suficientemente grande.

Para obter uma terceira solução, consideremos o sistema

$$
\begin{cases}-\Delta u=a u+b v+\left(-f_{1}(x,-v)+\left(-h_{1}(x)\right)\right) ; & \text { em } \Omega \\ -\Delta v=b u+a v+\left(-f_{2}(x,-u)+\left(-h_{2}(x)\right)\right) ; & \text { em } \Omega \\ u(x)=v(x)=0 ; & \text { in } \partial \Omega .\end{cases}
$$

Observemos que se $(u, v)$ é solução de (5.78), então $(-u,-v)$ é solução de (1.1 + ). Denotemos $g_{i}(x, t)=-f_{i}(x,-t) \mathrm{e}$

$$
G_{i}(x, t)=\int_{0}^{t} g_{i}(x, s) d s=\int_{0}^{t}-f_{i}(x,-s) d s=\int_{0}^{-t} f_{i}(x, \sigma) d \sigma=F_{i}(x,-t)
$$

para $i=1,2$. Segue que $g_{1}, g_{2}$ e $-h_{1},-h_{2}$ satisfazem (5.1) e (f) (ou (F) ).

Além disso, se $\widehat{J}_{a, b}$ é o funcional associado ao problema (5.78), então é fácil de verificar que $\widehat{J}_{a, b}(\mathbf{u})=J_{a, b}(-\mathbf{u})$, para todo $\mathbf{u} \in E$ : de fato a parte quadrática do funcional é par, enquanto $F_{i}(x, t)=G_{i}(x,-t)$, e $h_{i} t=\left(-h_{i}\right)(-t)$, para todo $t \in \mathbb{R}$ e $i=1,2$.

Então, podemos repetir a construção feita para obter $\widetilde{\mathbf{u}}^{j}$, obtendo uma sequência $\left\{\widehat{\mathbf{u}}^{j}\right\}_{j \in \mathbb{N}} \subset E$ e $j_{1} \in \mathbb{N}$, tais que para cada $j \geq j_{1}$, $\widehat{\mathbf{u}}^{j}$ é solução positiva de $(\underline{(5.78)})_{j}$ e $\widehat{J}_{a_{j}, b_{j}}\left(\widehat{\mathbf{u}}^{j}\right) \rightarrow-\infty$.

Concluímos que $-\widehat{\mathbf{u}}^{j}$ é uma solução negativa (logo distinta de $\left.\widetilde{\mathbf{u}}^{j}\right)$ de $(\underline{1.1}+)_{j}$, para $j \geq j_{1}$. Além disso, $J_{a_{j}, b_{j}}^{+}\left(-\widehat{\mathbf{u}}^{j}\right) \rightarrow-\infty$, implicando que $-\widehat{\mathbf{u}}^{j}$ é distinta também de $\mathbf{v}^{j}$.

Demonstração do Teorema 5.1. Item (a): Suponhamos que a afirmação do teorema seja falsa. Desta forma dado $\delta>0$, existiriam sequências $\left\{a_{j}\right\},\left\{b_{j}\right\}$ tais que $a_{j}-b_{j} \in$ $\left(\lambda_{l-1}+\delta, \lambda_{l}-\delta\right)\left(\right.$ ou $\left.a_{j}-b_{j} \in\left(-1 / \delta, \lambda_{1}-\delta\right)\right)$ e $a_{j}+b_{j} \rightarrow \lambda_{1}^{-}$, pelas quais o problema $(1.1+)_{j}$ não admite três soluções. Sem perda de generalidade, podemos supor $a_{j}+b_{j} \in\left(\lambda_{1}-\varepsilon_{j}, \lambda_{1}\right)$, mas isto seria uma contradição com a Proposição 5.4 .

Item (b): Pode ser demonstrado como no caso do Teorema 3.1, (veja Seção 3.2.3): as 
soluções serão pontos críticos do funcional

$$
J_{a, b}^{-}(\mathbf{u})=-1 / 2 B_{a, b}(\mathbf{u}, \mathbf{u})-(\mathcal{F}(\mathbf{u})+\mathcal{H}(\mathbf{u}))
$$

análogo a $J_{a, b}^{+}$exceto por ter a forma $-B_{a, b}$ no lugar de $B_{a, b}$.

O Lema 5.5 se estende facilmente ao caso $\lambda \in\left[\lambda_{1}, \lambda_{1}+\bar{\varepsilon}\right.$ ) (de fato, na prova nunca é usado o sinal de $\lambda-\lambda_{1}$ ) e os lemas seguintes se estendem por consequência.

Podemos então repetir a prova feita neste capítulo, definindo os espaços $V, Z, W$ como em (5.4), mas através dos autovalores de $-B_{a, b}$. Em particular, o autovalor correspondente a $\psi_{1}$ será

$$
0<\frac{(a+b)-\lambda_{1}}{\lambda_{1}}=-B_{a, b}\left(\boldsymbol{\psi}_{1}, \boldsymbol{\psi}_{1}\right)<\frac{\varepsilon}{\lambda_{1}},
$$

quando $a+b \in\left(\lambda_{1}, \lambda_{1}+\varepsilon\right)$. A positividade deste autovalor é o que nos permite demonstrar o Lema 5.9 (veja a equação (5.76) $)$.

Demonstração do Teorema 5.2. Análogo à prova do Teorema 3.2, do Capítulo 3 , 


\section{Apêndice \\ $\mathcal{A}$ \\ Conceitos de Análise Funcional}

Neste apêndice apresentamos algumas definições e resultados básicos da análise funcional, cuja principal referência é Bre83. Começamos definindo imersões contínuas e compactas de espaços normados.

Definição A.1. Sejam E e F espaços normados.

- Dizemos que E está continuamente imerso em $F$ e denotamos por $E \hookrightarrow F$, quando $E \subset F$ e existe $k \geq 0$, tal que $\|u\|_{F} \leq k\|u\|_{E}$ para todo $u \in E$;

- Dizemos que E está compactamente imerso em $F$ e denotamos por $E \stackrel{\text { cpto. }}{\longrightarrow} F$, quando $E \hookrightarrow F$, e se $x_{n} \rightarrow x$ em E, então passando a subsequência, $x_{n} \rightarrow x$ em $F$.

Com respeito a transformações lineares entre espaços de Banach utilizamos, no Capítulo 5, o Teorema do Gráfico Fechado.

Teorema A.2 (Teorema II.7 de [Bre83]). Sejam $X$ e $Y$ dois espaços de Banach. Se $T: X \rightarrow Y$ é uma transformação linear tal que $\overline{G(T)}=G(T) \subset X \times Y$, onde

$$
G(T)=\{(x, y) \in X \times Y: y=T x\},
$$

então $T$ é contínua, isto é, existe uma constante $c>0$, tal que

$$
\|T x\|_{Y} \leq c\|x\|_{X}
$$

para todo $x \in X$. 
Ainda no Capítulo 5, precisamos do Teorema de Egorov.

Teorema A.3 (Teorema IV.28 de Bre83]). Sejam $\Omega$ um subconjunto mensurável de $\mathbb{R}^{N}$ com medida finita e $\left\{f_{n}\right\}$ uma sequência de funções mensuráveis de $\Omega$ em $\mathbb{R}$. Se $f_{n}(x) \rightarrow$ $f(x)$ q. t. p. em $\Omega$, então para todo $\varepsilon>0$ existe um conjunto mensurável $A_{\varepsilon} \subseteq \Omega$, tal que $\left|\Omega \backslash A_{\varepsilon}\right|<\varepsilon$ e $f_{n} \rightarrow f$ uniformemente em $A_{\varepsilon}$.

\section{A.1 O espaço das funções continuamente diferenciáveis}

Consideremos o multi-índice $\alpha=\left(\alpha_{1}, \alpha_{2}, \ldots, \alpha_{n}\right)$, com $\alpha_{i} \geq 0$ inteiros, e definamos

$$
|\alpha|=\alpha_{1}+\alpha_{2}+\ldots+\alpha_{n} \quad \text { e } \quad D^{\alpha}=\partial^{|\alpha|} / \partial x_{1}^{\alpha_{1}} \partial x_{2}^{\alpha_{2}} \ldots \partial x_{n}^{\alpha_{n}} .
$$

Seja $\Omega \subseteq \mathbb{R}^{n}$. Definimos os conjuntos

$$
\mathcal{C}(\Omega)=\{u: \Omega \rightarrow \mathbb{R}: u \text { é contínua em } \Omega\},
$$

$\mathcal{C}^{k}(\Omega)\left\{u: \Omega \rightarrow \mathbb{R}: D^{\alpha} u\right.$ existe e é contínua em $\Omega, \forall \alpha$, tal que $\left.|\alpha| \leq k\right\}$,

$$
\mathcal{C}^{\infty}(\Omega)=\bigcap_{k \in \mathbb{N} \cup\{0\}} \mathcal{C}^{k}(\Omega)
$$

Ainda escrevemos $\mathcal{C}^{0}(\Omega)=\mathcal{C}(\Omega)$ e $\mathcal{C}_{c}^{k}(\Omega)$ representa as funções $\mathcal{C}^{k}(\Omega)$ com suporte compacto em $\Omega$.

Seja $\Omega \subset \mathbb{R}^{N}$ um subconjunto limitado. Então $\mathcal{C}(\bar{\Omega})$, munido com a norma

$$
\|u\|_{0}=\max _{x \in \Omega}|u(x)|, \quad \forall u \in \mathcal{C}(\bar{\Omega})
$$

é um espaço de Banach. De uma maneira mais geral, para todo $k \in \mathbb{N} \cup\{0\}$, o conjunto $\mathcal{C}^{k}(\bar{\Omega})$ munido da norma

$$
\|u\|_{k}=\sum_{|\alpha| \leq k}\left\|D^{\alpha} u\right\|_{0}, \quad \forall u \in \mathcal{C}^{k}(\bar{\Omega})
$$

é um espaço de Banach. 


\section{A.2 Os espaços de Hölder}

Nesta seção definimos os espaços de Hölder e enunciamos alguns resultados clássicos. Para mais detalhes ver, por exemplo, GT01.

Definição A.4. Dado $0<\gamma \leq 1$ e uma função $u \in \mathcal{C}(\Omega)$, dizemos que u é Hölder continua com expoente $\gamma$, se existe uma constante $c>0$, tal que

$$
|u(x)-u(y)| \leq c|x-y|^{\gamma}, \quad \forall x, y \in \Omega
$$

Para uma tal função definimos o quociente de Hölder por

$$
H_{\gamma}[u]:=\sup _{x, y \in \Omega} \frac{|u(x)-u(y)|}{|x-y|^{\gamma}}<\infty
$$

O conjunto

$$
\mathcal{C}^{0, \gamma}(\bar{\Omega})=\left\{u \in \mathcal{C}(\bar{\Omega}): H_{\gamma}[u]<\infty\right\}
$$

é um espaço de Banach com a norma

$$
\|u\|_{0, \gamma}=\|u\|_{0}+H_{\gamma}[u]
$$

De uma maneira mais geral, temos a seguinte definição.

Definição A.5. Seja $k \in \mathbb{N} \cup\{0\}$ e $0<\gamma \leq 1$. O espaço de Hölder $\mathcal{C}^{k, \gamma}(\bar{\Omega})$ é definido por

$$
\mathcal{C}^{k, \gamma}(\bar{\Omega})=\left\{u \in \mathcal{C}^{k}(\bar{\Omega}): H_{\gamma}\left[D^{\alpha} u\right]<\infty \text { para todo multi-índice }|\alpha| \leq k\right\}
$$

Definamos ainda

$$
\mathcal{C}^{k, \gamma}(\Omega)=\left\{u \in \mathcal{C}^{k}(\Omega): u \in \mathcal{C}^{k, \gamma}\left(\bar{\Omega}_{0}\right) \text { para todo } \Omega_{0} \subset \subset \Omega\right\}
$$

Como antes, o conjunto $\mathcal{C}^{k, \gamma}(\bar{\Omega})$ é um espaço de Banach, quando munido da norma

$$
\|u\|_{k, \gamma}=\|u\|_{k}+\sum_{|\alpha| \leq k} H_{\gamma}\left[D^{\alpha} u\right], \quad \forall u \in \mathcal{C}^{k, \gamma}(\bar{\Omega}) .
$$

Vale o teorema. 
Teorema A.6. Sejam $0<\nu<\gamma \leq 1$ e $k \in \mathbb{N} \cup\{0\}$.

- Seja $\Omega \subset \mathbb{R}^{N}$ aberto, então

$-\mathcal{C}^{k+1}(\bar{\Omega}) \hookrightarrow \mathcal{C}^{k}(\bar{\Omega})$,

- $\mathcal{C}^{k, \gamma}(\bar{\Omega}) \hookrightarrow \mathcal{C}^{k}(\bar{\Omega})$ (a imersão também é compacta se $\Omega$ é limitado),

- $\mathcal{C}^{k, \gamma}(\bar{\Omega}) \hookrightarrow \mathcal{C}^{k, \nu}(\bar{\Omega})$ (a imersão também é compacta se $\Omega$ é limitado).

- Se $\Omega$ é convexo, então

$$
\begin{aligned}
& -\mathcal{C}^{k+1}(\bar{\Omega}) \hookrightarrow \mathcal{C}^{k, 1}(\bar{\Omega}) \text { (vale também } \mathcal{C}^{k+1}(\bar{\Omega}) \stackrel{\text { cpto. }}{\longrightarrow} \mathcal{C}^{k}(\bar{\Omega}) \text {, se } \Omega \text { é limitado), } \\
& -\mathcal{C}^{k+1}(\bar{\Omega}) \hookrightarrow \mathcal{C}^{k, \nu}(\bar{\Omega}) \text { (a imersão também é compacta se } \Omega \text { é limitado). }
\end{aligned}
$$

\section{A.3 Os espaços $L^{p}$}

Nesta seção definimos e apresentamos alguns resultados com respeito aos espaços $L^{p}(\Omega)$.

Seja $\Omega \subseteq \mathbb{R}^{N}$ um conjunto aberto e $\partial \Omega$ sua fronteira. Para $p \in[1,+\infty)$, seja

$$
\widetilde{L}^{p}(\Omega)=\left\{u: \Omega \rightarrow \mathbb{R}, \text { tais que } \int_{\Omega}|u|^{p}<+\infty\right\}
$$

e definamos $L^{p}(\Omega)$ como o conjunto das classes de equivalência de elementos de $\widetilde{L}^{p}(\Omega)$ que são iguais q. t. p., munido com a norma $\|u\|_{L^{p}}=\left(\int_{\Omega}|u|^{p}\right)^{\frac{1}{p}}$.

Além disso, seja

$$
\widetilde{L}^{\infty}(\Omega)=\{u: \Omega \rightarrow \mathbb{R} \text {, mensurável, tal que } \exists c>0:|u|<c \text {, q. t. p. em } \Omega\},
$$

e definamos como acima $L^{\infty}(\Omega)$ munido com a norma

$$
\|u\|_{L^{\infty}}=\inf \{c:|u|<c, \text { q. t. p. em } \Omega\} \text {. }
$$

Sabemos que

- $\left(L^{p}(\Omega),\|\cdot\|_{L^{p}}\right)$ é um Espaço de Banach para $p \in[1,+\infty]$, e é Espaço de Hilbert se $p=2$ com produto interno $\langle u, v\rangle_{L^{2}}=\int_{\Omega} u v$. 
- $L^{p}(\Omega)$ é reflexivo e separável para $p \in(1,+\infty)$, e seu dual é $L^{q}(\Omega)$, com $\frac{1}{p}+\frac{1}{q}=1$.

- $L^{1}(\Omega)$ é separável enquanto $L^{\infty}(\Omega)$ não é. O dual de $L^{1}(\Omega)$ é $L^{\infty}(\Omega)$, enquanto o dual de $L^{\infty}(\Omega)$ contém $L^{1}(\Omega)$. Nenhum dos dois espaços é reflexivo.

Teorema A.7 (Desigualdade de Hölder). Sejam $f \in L^{p}(\Omega)$ e $g \in L^{q}(\Omega)$, onde $\frac{1}{p}+\frac{1}{q}=1$ para $p, q \in(1,+\infty)$ (vale também para $q=1$ e $p=\infty$, ou $q=\infty$ e $p=1$ ). Então $f g \in L^{1} e$

$$
\int_{\Omega}|f g| \leq\|f\|_{L^{p}}\|g\|_{L^{q}}
$$

Lema A.8. Seja $\Omega$ um subconjunto aberto e limitado de $\mathbb{R}^{N}$. Se $1 \leq p \leq q \leq \infty$, então $L^{q}(\Omega)$ está continuamente imerso em $L^{p}(\Omega)$, isto é, $L^{q}(\Omega) \subset L^{p}(\Omega)$ e existe uma constante $C>0$, dependendo de $\Omega$, tal que

$$
\|u\|_{L^{p}} \leq C\|u\|_{L^{q}}, \quad \text { para todo } u \in L^{q}(\Omega) .
$$

Por fim, demonstramos um lema que contém desigualdades bastante úteis para relacionar a norma $L^{p}$ com a norma do produto cartesiano $L^{p} \times L^{p}$.

Lema A.9. Se $a, b \geq 0$ e $p \in[1,+\infty)$, então

$$
a^{p}+b^{p} \leq(a+b)^{p} \leq 2^{p-1}\left(a^{p}+b^{p}\right) .
$$

Demonstração. Se $a=0$ ou $b=0$ a prova é trivial. Portanto, suponhamos $a$ e $b$ positivos. Definamos a função $f: \mathbb{R}_{+} \rightarrow \mathbb{R}$, dada por

$$
f(x)=\frac{(1+x)^{p}}{\left(1+x^{p}\right)}
$$

Assim $f(0)=1, \lim _{x \rightarrow+\infty} f(x)=1, f(x)>1$, para todo $x \in(0, \infty)$ e $f$ atinge máximo em $x=1$. Portanto $\operatorname{Im}(f) \subseteq[1, f(1)]=\left[1,2^{p-1}\right]$. Assim, tomando $x=\frac{b}{a}$, temos

$$
1 \leq \frac{\left(1+\frac{b}{a}\right)^{p}}{1+\frac{b^{p}}{a^{p}}} \leq 2^{p-1} \Longleftrightarrow 1 \leq \frac{(a+b)^{p}}{a^{p}+b^{p}} \leq 2^{p-1}
$$

que é equivalente a (A.1). 


\section{A.4 Os espaços de Sobolev}

Introduzimos os espaços de Sobolev e apresentamos alguns dos principais resultados que utilizamos nesta tese.

Sejam $\Omega \subset \mathbb{R}^{N}$ um aberto e $p \in \mathbb{R}$ com $1 \leq p \leq \infty$. Consideremos o multi-índice $\alpha=\left(\alpha_{1}, \alpha_{2}, \ldots, \alpha_{n}\right)$.

Definição A.10. Sejam $m>0$ inteiro positivo e $1 \leq p \leq \infty$. O espaço de Sobolev $W^{m, p}(\Omega)$ é definido como

$W^{m, p}(\Omega)=\left\{u \in L^{p}(\Omega): \forall \alpha \operatorname{com}|\alpha| \leq m, \exists D^{\alpha} u \in L^{p}(\Omega)\right.$, no sentido das distribuições $\}$,

com a norma

$$
\|u\|_{W^{k, p}}=\left(\sum_{|\alpha| \leq k}\left\|D^{\alpha} u\right\|_{L^{p}}^{p}\right)^{1 / p}, \text { se } 1 \leq p<\infty
$$

respectivamente, com norma

$$
\|u\|_{W^{k, \infty}}=\max _{|\alpha| \leq k}\left\|D^{\alpha} u\right\|_{L^{\infty}}
$$

Quando $p=2$, geralmente utilizamos a notação $H^{k}(\Omega)=W^{k, 2}(\Omega)$. Sabemos que

- $\left(W^{k, p}(\Omega),\|\cdot\|_{W^{k, p}}\right)$ é um espaço de Banach para $p \in[1,+\infty]$, e é espaço de Hilbert quando $p=2$, com o produto escalar $\langle u, v\rangle_{W^{k, 2}}=\sum_{|\alpha| \leq k} \int_{\Omega} D^{\alpha} u D^{\alpha} v$.

- $W^{k, p}(\Omega)$ é reflexivo e separável para $p \in(1,+\infty)$, mas apenas separável para $p=1$.

Definição A.11. O espaço $W_{0}^{1, p}(\Omega)$ é definido como o fecho de $\mathcal{C}_{c}^{1}(\Omega)$ na norma $W^{1, p}(\Omega)$, munido com esta mesma norma.

Se $\Omega$ for de classe $\mathcal{C}^{1}$ (ver Definição 2.7) e $u \in W^{1, p}(\Omega) \cap \mathcal{C}(\bar{\Omega})$, para $1 \leq p<\infty$, vale que $u \in W_{0}^{1, p}(\Omega)$ se, e somente se, $u \in W^{1, p}(\Omega)$ e $u(x)=0$, para $x \in \partial \Omega$. Temos também que os espaços $W_{0}^{1, p}$ possuem as mesmas propriedades, no que diz respeito a separabilidade e reflexividade, dos correspondentes espaços $W^{1, p}(\Omega)$. Ainda, o espaço $W_{0}^{1,2}(\Omega)$ é de Hilbert e é denotado por $H_{0}^{1}(\Omega)$.

Uma outra propriedade importante desses espaços é a Desigualdade de Poincaré. 
Teorema A.12 (Desigualdade de Poincaré). Se $\Omega$ é limitado, então existe uma constante $C>0$, dependendo de $p$ e $\Omega$, tal que $\|u\|_{L^{p}} \leq C\|\nabla u\|_{L^{p}}$, para todo $u \in W_{0}^{1, p}(\Omega)$.

Este teorema implica que em $W_{0}^{1, p}(\Omega),\|\nabla u\|_{L^{p}}$ é uma norma equivalente a $\|u\|_{W^{1, p}}$.

Listamos agora os resultados conhecidos como imersões de Sobolev. Estaremos supondo que o conjunto $\Omega$ seja de classe $\mathcal{C}^{1}$ (ver Definição 2.7). Para mais detalhes ver por exemplo [Bre83, Str90].

- Para $\frac{1}{p}-\frac{m}{N}>0$, seja $p^{*}=p^{*}(m, p, N)=\left(\frac{1}{p}-\frac{m}{N}\right)^{-1}$. Temos $W^{m, p}(\Omega) \subset L^{q}(\Omega)$

- para todo $q \in\left[p, p^{*}\right]$ com inclusão contínua, se $\Omega$ é ilimitado,

- para todo $q \in\left[1, p^{*}\right]$ com inclusão contínua e também compacta (exceto para $\left.q=p^{*}\right)$, se $\Omega$ é limitado.

- Para $\frac{1}{p}-\frac{m}{N}=0$, temos $W^{m, p}(\Omega) \subset L^{q}(\Omega)$

- para todo $q \in[p,+\infty)$ com inclusão contínua, se $\Omega$ é ilimitado,

- para todo $q \in[1,+\infty)$ com inclusão contínua e compacta, se $\Omega$ é limitado.

- Para $\frac{1}{p}-\frac{m}{N}<0$, temos $W^{m, p}(\Omega) \subset L^{q}(\Omega)$

- para todo $q \in[p,+\infty)$ com inclusão contínua, se $\Omega$ é ilimitado,

- para todo $q \in[1,+\infty)$ com inclusão contínua e compacta, se $\Omega$ é limitado.

Além disso, se $k$ e $m$ são respectivamente, as partes inteira e decimal de $m-\frac{N}{p}$, temos:

- se $\alpha \neq 0$, então $W^{m, p} \subset \mathcal{C}^{k, \alpha}(\bar{\Omega})$ com inclusão contínua (também compacta se $\Omega$ é limitado),

- se $\alpha=0$, então $W^{m, p} \subset \mathcal{C}^{k-1,1}(\bar{\Omega})$ com inclusão contínua (também compacta se $\Omega$ é limitado).

Observemos que nos casos em que a imersão $W^{m, p}(\Omega) \subset L^{q}(\Omega)$ é compacta e $p \in$ $(1,+\infty)$ (portanto $W^{m, p}(\Omega)$ é reflexivo e separável), temos que se $\left\{u_{n}\right\}$ é uma sequência limitada em $W^{m, p}(\Omega)$, então existe $u \in W^{m, p}(\Omega)$, tal que passando a subsequência

$$
\left\{\begin{array}{l}
u_{n} \rightarrow u, \quad \text { em } W^{m, p}(\Omega), \\
u_{n} \rightarrow u, \quad \text { em } L^{q}(\Omega) .
\end{array}\right.
$$


Em particular, quando $\Omega$ é limitado, se $\left\{u_{n}\right\} \subset H^{1}(\Omega)$ é limitada, então, a menos de subsequência, $u_{n} \rightarrow u$ em $H^{1}(\Omega)$ e $u_{n} \rightarrow u$ em $L^{s}(\Omega)$, para todo $s \in\left[1, \frac{2 n}{n-2}\right)$ e algum $u \in H^{1}(\Omega)$. 


\section{Apêndice $\mathcal{B}$ \\ Operadores de Nemytskii}

Neste apêndice definimos os chamados Operadores de Nemytskii e apresentamos uma propriedade que precisamos nos Capítulos 3 e 5 .

Sejam $\Omega$ um subconjunto aberto de $\mathbb{R}^{N}$ e $\mathcal{M}(\Omega)$ o conjunto de todas as funções Lebesgue mensuráveis $u: \Omega \rightarrow \mathbb{R}$.

Definição B.1. Seja $\Omega$ um subconjunto aberto de $\mathbb{R}^{N}$. Uma função $f: \Omega \times \mathbb{R} \rightarrow \mathbb{R}$ é dita função de Carathéodory quando possui as propriedades:

(i) para cada $s \in \mathbb{R}$ fixado, a função $x \mapsto f(x, s)$ é Lebesgue mensurável em $\Omega$;

(ii) para quase todo $x \in \mathbb{R}$, a função $s \mapsto f(x, s)$ é contínua em $\mathbb{R}$.

Sobre as funções de Carathéodory, temos a seguinte propriedade:

Teorema B.2 (Teorema 2.1 de [dF89]). Seja $f: \Omega \times \mathbb{R} \rightarrow \mathbb{R}$ uma função de Carathéodory. Para todo $u \in \mathcal{M}$, a função $x \mapsto f(x, u(x))$ é mensurável.

Com isto, uma função de Carathéodory $f$ define o operador

$$
N_{f}: \mathcal{M} \rightarrow \mathcal{M}: u \mapsto f(\cdot, u(\cdot))
$$

chamado Operador de Nemytskii associado a $f$. Em [Bre83] e dF89], são demonstradas várias propriedades destes operadores. Entre elas, resultados que determinam condições sobre a função $f$ para que o operador $N_{f}$ esteja bem definido e seja contínuo, de $L^{p}(\Omega)$ em 
$L^{r}(\Omega)$ para $1 \leq p, r \leq \infty$. Enunciamos aqui um resultado, que será utilizado no capítulo 3. a respeito da continuidade de tal operador no caso em que $\Omega$ é limitado.

Teorema B.3. [Teorema 2.5 de [dF89]] Sejam $\Omega$ um dominio limitado, $1 \leq p, r<\infty$, $f$ uma função de Carathéodory e $N_{f}$ o operador de Nemytskii associado à função $f$. Suponhamos que $N_{f}$ transforme funções de $L^{p}(\Omega)$ em funções $L^{r}(\Omega)$. Então $N_{f}$ é operador contínuo e limitado, quando é considerado como um operador de $L^{p}(\Omega)$ em $L^{r}(\Omega)$. 
Apêndice

C

Diferenciabilidade de funcionais em espaços de Banach

Seja $E: \Omega \times \mathbb{R} \times \mathbb{R}^{N} \rightarrow \mathbb{R}$. Neste apêndice discutimos a diferenciabilidade de funcionais do tipo

$$
J(u)=\int_{\Omega} E(x, u(x), \nabla u(x)) d x
$$

onde $u \in H_{0}^{1}(\Omega)$ e $\Omega \subseteq \mathbb{R}^{N}$ um aberto. Começamos definindo o conceito de diferenciabilidade de Fréchet.

Definição C.1. Um funcional J definido num espaço de Banach $X$ é Fréchet diferenciável (ou diremos apenas diferenciável) num ponto $u \in X$, se existir uma aplicação linear limitada $D J(u) \in X$, chamada de diferencial de $J$ em $u$, tal que

$$
\frac{|J(u+v)-J(u)-D J(u)[v]|}{\|v\|_{X}} \rightarrow 0, \quad \text { quando } \quad\|v\|_{X} \rightarrow 0 .
$$

O funcional $J$ é dito de classe $\mathcal{C}^{1}(X, \mathbb{R})$ (ou apenas $\mathcal{C}^{1}$ ) se a aplicação $u \mapsto D J(u)$ é continua.

As propriedades de diferenciabilidade do funcional $J$ dependem crucialmente das condições de crescimento de E. É o que observamos no seguinte Teorema.

Teorema C.2 (Teorema C.1 de [Str90]). Seja $E: \Omega \times \mathbb{R} \times \mathbb{R}^{N} \rightarrow \mathbb{R}$ uma função mensurável em $x \in \Omega$, continuamente diferenciável em $u \in \mathbb{R}$ e em $p \in \mathbb{R}^{N}$, e que satisfaça as 
seguintes condições de crescimento, onde $E_{u}=\frac{\partial}{\partial u} E, E_{p}=\frac{\partial}{\partial p} E$,

(1) $|E(x, u, p)| \leq C\left(1+|u|^{s_{1}}+|p|^{2}\right)$, onde $s_{1}<\frac{2 N}{N-2}$, se $N \geq 3$,

(2) $\left|E_{u}(x, u, p)\right| \leq C\left(1+|u|^{s_{2}}+|p|^{t_{2}}\right)$, onde $t_{2} \leq 2$, se $N \leq 2, s_{2} \leq \frac{N+2}{N-2}, t_{2} \leq \frac{N+2}{N}$, se $N \geq 3$

(3) $\left|E_{p}(x, u, p)\right| \leq C\left(1+|u|^{s_{3}}+|p|\right)$, onde $s_{3} \leq \frac{N}{N-2}$, se $N \geq 3$.

Então (C.1) define um funcional de classe $\mathcal{C}^{1}\left(H_{0}^{1}(\Omega), \mathbb{R}\right)$.

Observemos que os funcionais definidos nos Capítulos 3, 4 e 5 estão definidos no produto $H_{0}^{1}(\Omega) \times H_{0}^{1}(\Omega)$, e portanto não são do tipo (C.1). No entanto podemos considerar, para cada $v \in H_{0}^{1}(\Omega)$ fixado, o funcional

$$
\begin{aligned}
J_{v}^{+}: H_{0}^{1}(\Omega) & \rightarrow \mathbb{R} \\
u & \mapsto J_{v}^{+}(u)=J^{+}(u, v),
\end{aligned}
$$

e, analogamente, para cada $u \in H_{0}^{1}(\Omega)$ fixado

$$
\begin{aligned}
J_{u}^{+}: H_{0}^{1}(\Omega) & \rightarrow \mathbb{R} \\
v & \mapsto J_{u}^{+}(v)=J^{+}(u, v) .
\end{aligned}
$$

Ambos os funcionais são de classe $\mathcal{C}^{1}\left(H_{0}^{1}(\Omega), \mathbb{R}\right)$, em vista do Teorema C.2 e da condição (1.2).

Segue então que $J^{+}$é de classe $\mathcal{C}^{1}\left(H_{0}^{1}(\Omega) \times H_{0}^{1}(\Omega), \mathbb{R}\right)$, sendo sua diferencial dada por $D J^{+}(u, v)[(\phi, \psi)]=D J_{v}^{+}(u)[\phi]+D J_{u}^{+}(v)[\psi]$. 


\section{Índice}

Alternativa

de Fredholm, 15

autofunção, 14

autovalor, 14

condição

de Palais-Smale, 11

desigualdade

de Hölder, 93

de Poincaré, 95

espaço

$L^{\infty}, 92$

$L^{p}, 92$

$\mathcal{C}^{\infty}, 90$

$\mathcal{C}^{k}, 90$

de Hölder, 91

de Sobolev, 94

espectro

do operador Laplaciano, 14

função

de Carathéodary, 97

Hölder contínua, 91

funcional

fortemente indefinido, 17
Fréchet diferenciável, 99

imersão

compacta, 89

contínua, 89

de Sobolev, 95

Lema

de Deformação, 11

Método

de Galerkin, 18

multi-índice, 90

multiplicidade

do autovalor, 14

operador

de Nemytskii, 97

quociente

de Hölder, 91

sequência

de Palais-Smale, 11

sistema

de tipo gradiente, 16

de tipo hamiltoniano, 17

solução 
clássica, 9

fraca, 9

Teorema

do Passo da Montanha, 12

do Ponto de Sela, 13

de Egorov, 90

do Gráfico Fechado, 89 


\section{Bibliografia}

[ADN59] S. Agmon, A. Douglis, and L. Nirenberg, Estimates near the boundary for solutions of elliptic partial differential equations satisfying general boundary conditions. I, Comm. Pure Appl. Math. 12 (1959), 623-727.

[Agm59] S. Agmon, The $L_{p}$ approach to the Dirichlet problem. I. Regularity theorems, Ann. Scuola Norm. Sup. Pisa (3) 13 (1959), 405-448.

[AM07] A. Ambrosetti and A. Malchiodi, Nonlinear analysis and semilinear elliptic problems, Cambridge Studies in Advanced Mathematics, vol. 104, Cambridge University Press, Cambridge, 2007.

[AR73] A. Ambrosetti and P. H. Rabinowitz, Dual variational methods in critical point theory and applications, J. Functional Analysis 14 (1973), 349-381.

[AS12] Yu-C. An and H.-M. Suo, Multiple solutions for degenerate elliptic systems near resonance at higher eigenvalues, Abstr. Appl. Anal. (2012), Art. ID 532430, 19.

[BBF83] P. Bartolo, V. Benci, and D. Fortunato, Abstract critical point theorems and applications to some nonlinear problems with "strong" resonance at infinity, Nonlinear Anal. 7 (1983), no. 9, 981-1012.

[BL89] M. Badiale and D. Lupo, Some remarks on a multiplicity result by Mawhin and Schmitt, Acad. Roy. Belg. Bull. Cl. Sci. (5) 65 (1989), no. 6-9, 210-224. 
[Bre83] H. Brezis, Analyse fonctionnelle, Collection Mathématiques Appliquées pour la Maîtrise. [Collection of Applied Mathematics for the Master's Degree], Masson, Paris, 1983, Théorie et applications. [Theory and applications].

[CdF93] R. Chiappinelli and D. G. de Figueiredo, Bifurcation from infinity and multiple solutions for an elliptic system, Differential Integral Equations 6 (1993), no. $4,757-771$.

[CdFM92] Ph. Clément, D. G. de Figueiredo, and E. Mitidieri, Positive solutions of semilinear elliptic systems, Comm. Partial Differential Equations 17 (1992), no. 5-6, 923-940.

[CMN92] R. Chiappinelli, J. Mawhin, and R. Nugari, Bifurcation from infinity and multiple solutions for some Dirichlet problems with unbounded nonlinearities, Nonlinear Anal. 18 (1992), no. 12, 1099-1112.

[dF89] D. G. de Figueiredo, Lectures on the Ekeland variational principle with applications and detours, Tata Institute of Fundamental Research Lectures on Mathematics and Physics, vol. 81, Published for the Tata Institute of Fundamental Research, Bombay, 1989.

[dF00] D. G. de Figueiredo, Nonlinear elliptic systems, An. Acad. Brasil. Ciênc. 72 (2000), no. 4, 453-469.

[dF08] D. G. de Figueiredo, Semilinear elliptic systems: existence, multiplicity, symmetry of solutions, Handbook of differential equations: stationary partial differential equations. Vol. V, Handb. Differ. Equ., Elsevier/North-Holland, Amsterdam, 2008, pp. 1-48.

[dFdÓR04] D. G. de Figueiredo, J. M. do Ó, and B. Ruf, Critical and subcritical elliptic systems in dimension two, Indiana Univ. Math. J. 53 (2004), no. 4, 10371054.

[DNM01] P. De Nápoli and M. C. Mariani, Three solutions for quasilinear equations in $\mathbf{R}^{n}$ near resonance, Proceedings of the USA-Chile Workshop on Nonlinear Analysis (Viña del Mar-Valparaiso, 2000) (San Marcos, TX), Electron. J. Differ. Equ. Conf., vol. 6, Southwest Texas State Univ., 2001, pp. 131-140. 
[dPM08] F. O. de Paiva and E. Massa, Semilinear elliptic problems near resonance with a nonprincipal eigenvalue, J. Math. Anal. Appl. 342 (2008), no. 1, 638-650.

[Eva98] L. C. Evans, Partial differential equations, Graduate Studies in Mathematics, vol. 19, American Mathematical Society, Providence, RI, 1998.

[Fel93] Patricio L. Felmer, Periodic solutions of "superquadratic" Hamiltonian systems, J. Differential Equations 102 (1993), no. 1, 188-207.

[GT01] D. Gilbarg and N. S. Trudinger, Elliptic partial differential equations of second order, Classics in Mathematics, Springer-Verlag, Berlin, 2001, Reprint of the 1998 edition.

[HvdV93] J. Hulshof and R. van der Vorst, Differential systems with strongly indefinite variational structure, J. Funct. Anal. 114 (1993), no. 1, 32-58.

[KT11a] X.-F. Ke and C.-L. Tang, Existence and multiplicity of solutions for asymptotically linear noncooperative elliptic systems, J. Math. Anal. Appl. 375 (2011), no. $2,631-647$.

[KT11b] X.-F. Ke and C.-L. Tang, Multiple solutions for semilinear elliptic equations near resonance at higher eigenvalues, Nonlinear Anal. 74 (2011), no. 3, 805813.

[LR90] D. Lupo and M. Ramos, Some multiplicity results for two-point boundary value problems near resonance, Rend. Sem. Mat. Univ. Politec. Torino 48 (1990), no. 2, 125-135 (1992).

[Mas07] E. Massa, Multiplicity results for a superlinear elliptic system with partial interference with the spectrum, Nonlinear Anal. 67 (2007), no. 1, 295-306.

[MMP94] A. Marino, A. M. Micheletti, and A. Pistoia, A nonsymmetric asymptotically linear elliptic problem, Topol. Methods Nonlinear Anal. 4 (1994), no. 2, 289339.

[MP02] To Fu Ma and M. L. Pelicer, Perturbations near resonance for the p-Laplacian in $\mathbb{R}^{N}$, Abstr. Appl. Anal. 7 (2002), no. 6, 323-334. 
[MR06] E. Massa and B. Ruf, On the Fučik spectrum for elliptic systems, Topol. Methods Nonlinear Anal. 27 (2006), no. 2, 195-228.

[MRS97] To Fu Ma, M. Ramos, and L. Sanchez, Multiple solutions for a class of nonlinear boundary value problems near resonance: a variational approach, Proceedings of the Second World Congress of Nonlinear Analysts, Part 6 (Athens, 1996), vol. 30, 1997, pp. 3301-3311.

[MS90] J. Mawhin and K. Schmitt, Nonlinear eigenvalue problems with the parameter near resonance, Ann. Polon. Math. 51 (1990), 241-248.

[OT09] Z.-Q. Ou and C.-L. Tang, Existence and multiplicity results for some elliptic systems at resonance, Nonlinear Anal. 71 (2009), no. 7-8, 2660-2666.

[Pal63] R. S. Palais, Morse theory on Hilbert manifolds, Topology 2 (1963), 299-340.

[PS64] R. S. Palais and S. Smale, A generalized Morse theory, Bull. Amer. Math. Soc. 70 (1964), 165-172.

[Rab86] P. H. Rabinowitz, Minimax methods in critical point theory with applications to differential equations, CBMS Regional Conference Series in Mathematics, vol. 65, Published for the Conference Board of the Mathematical Sciences, Washington, DC, 1986.

[RS97] M. Ramos and L. Sanchez, A variational approach to multiplicity in elliptic problems near resonance, Proc. Roy. Soc. Edinburgh Sect. A 127 (1997), no. 2, $385-394$.

[Sma64] S. Smale, Morse theory and a non-linear generalization of the Dirichlet problem, Ann. of Math. (2) 80 (1964), 382-396.

[ST01] J. Su and C. Tang, Multiplicity results for semilinear elliptic equations with resonance at higher eigenvalues, Nonlinear Anal. 44 (2001), no. 3, Ser. A: Theory Methods, 311-321.

[ST10] H.-M. Suo and C.-L. Tang, Multiplicity results for some elliptic systems near resonance with a nonprincipal eigenvalue, Nonlinear Anal. 73 (2010), no. 7, 1909-1920. 
[Str90] M. Struwe, Variational methods, Springer-Verlag, Berlin, 1990, Applications to nonlinear partial differential equations and Hamiltonian systems.

[Wil96] M. Willem, Minimax theorems, Progress in Nonlinear Differential Equations and their Applications, 24, Birkhäuser Boston Inc., Boston, MA, 1996. 